\title{
Explorations of a family of stochastic Newmark methods in engineering dynamics
}

\author{
D. Roy ${ }^{\mathrm{a}, *}$, M.K. Dash ${ }^{\mathrm{b}}$ \\ ${ }^{a}$ Structural Engineering Division, Department of Civil Engineering, Indian Institute of Science, Bangalore 560012 , India \\ ${ }^{\mathrm{b}}$ Civil Engineering, Indira Gandhi Institute of Technology, Sarang, Dhenkanal, Orissa 759146, India
}

\begin{abstract}
A family of stochastic Newmark methods are explored for direct (path-wise or strong) integrations of stochastically driven dynamical systems of engineering interest. The stochastic excitations are assumed to be modeled by white noise processes or their filters and may be applied additively or multiplicatively. The family of stochastic Newmark maps are developed through a two-parameter, implicit Ito-Taylor expansion of the displacement and velocity vectors associated with the governing stochastic differential equations (SDE-s). Detailed estimates of local and global error orders for the response variables are provided in terms of the given time step size, $h$. While higher order Newmark methods lead to higher accuracies, far less random variables need to be modeled in the lower order methods to make it much more attractive from a computational point of view. For the specific case of a linear dynamical system, the stochastic Newmark map is used to obtain a closed form map for computing the temporal evolution of the response co-variance matrix. A host of numerical illustrations, covering linear and non-linear, single- and multi-degree-of-freedom dynamical systems, are provided to bring out the advantages and possible weaknesses of the methods proposed.
\end{abstract}

(C) 2005 Elsevier B.V. All rights reserved.

Keywords: Wiener processes; Stochastic differential equations; Ito-Taylor expansions; Path-wise solutions; Stochastic Newmark maps; Mechanical oscillators

\section{Introduction}

Newmark method is by far the most popular tool for direct integration of deterministic dynamical systems $[12,1,11,25]$. For a given time step size, $h$, a Newmark map may be derived by expanding the

\footnotetext{
* Corresponding author. Tel.: +91 3222 283450; fax: +91 3222255303.

E-mail addresses: royd@civil.iisc.ernet.in (D. Roy), manoj-orin@yahoo.co.uk (M.K. Dash).
} 
displacement and velocity components up to $\mathrm{O}\left(h^{2}\right)$ and $\mathrm{O}(h)$ respectively via a two-parameter implicit Taylor series. This corresponds to a constant average acceleration for a sufficiently continuous and differentiable deterministic system. The same logic, however, cannot be directly used for stochastic systems under white noise inputs. It is well known that a white noise process, defined formally as the derivative of a Wiener process, is not a valid mathematical or physical function of time. This is because a Wiener process, even though continuous, may have an unbounded variation over any given time interval. Consequently, for such engineering systems, the acceleration vector does not exist mathematically (at least, in the sense of sample paths). Moreover, increments of a Wiener process change by $\mathrm{O}\left(h^{1 / 2}\right)$ over a time interval of $h$. These two non-deterministic aspects of a stochastic differential equation (SDE) explain why numerical integration techniques for strong solutions of SDEs are often so different from their deterministic counterparts [4]. A consistent way to achieve higher order accuracy while developing numerical algorithms for pathwise (strong) solutions of SDEs is to use a stochastic Taylor expansion [4], which in turn is derivable from repeated applications of Ito-Taylor or Ito-Stratonovich formulas to a functional [6]. Direct integration methods provide an attractive numerical approach due to their simplicity and applicability to MDOF non-linear systems. A stochastic central difference method [18-21], and a Newmark family of algorithms [22] have been proposed for systems described by second-order matrix differential equations. Although successful, these methods are limited to non-linear elastic structures subjected to additive white noise excitations. Zhang and Zhao [24] updated the algorithm to correctly handle autocorrelation functions of response processes under Gaussian white noise excitations in a discrete form and clarified the effect of the time interval on the calculations. To represent a filtered white noise excitation, an AR model [8] and an ARMA model [32] were incorporated into the algorithms. Ohtori et al. [13] proposed a semi-implicit integration algorithm for stochastic analysis of state-space equations. It solves the linearized equations by an implicit method termed as "semi-implicit integration". By taking the mathematical expectation of the recursive expression for the solution of the state-space equation, the algorithm calculates the mean and covariance of the response of the structure. Bernard and Fleury [2] have recently proposed a stochastic Newmark scheme for solution of dynamical equation of linear oscillators subjected to white noise excitation. Runge-Kutta schemes in the strong sense have been proposed, for instance, by McShane [27], Rumelin [26], Chang [29], Milstein [9], Kloeden and Platen [7] and the references of Saito and Mitsui [28]. Tocino and Ardanuy [23] have proposed a class of explicit second order and two explicit third order RK schemes. A symplectic stochastic integrator [10] and a second order leapfrog algorithm [14] are also available in the literature.

The central purpose of this paper is to theoretically develop and numerically implement a family of lower and higher order stochastic Newmark algorithms for pathwise integration of SDE-s of relevance in stochastic structural dynamics. At the outset, certain rather general continuity and boundedness requirements are imposed on the drift and diffusion vectors. Secondly, lower and higher order stochastic Newmark maps are derived based on appropriate stochastic Taylor expansions of displacement and velocity vectors in terms of a given time step size, $h$. As in the deterministic case, the displacement and velocity expansions are performed implicitly using a couple of arbitrary integration parameters, $\alpha$ and $\beta$. Given a time step size $h$, the displacement and velocity expansions are computed by retaining terms up to $\mathrm{O}\left(h^{2}\right)$ and $\mathrm{O}(h)$ respectively in the lower order and terms up to $\mathrm{O}\left(h^{3}\right)$ and $\mathrm{O}\left(h^{2}\right)$ in the higher order. These expansions are not complete in the sense that certain terms of the highest order in $h$, involving multiple stochastic integrals (MSI-s) in terms of the Wiener increments, are dropped off from the expansions for the sake of computational convenience. Rigorous error estimates are carried out to determine the local and global error orders for displacement and velocity vectors. Systematic procedures for the determination of MSI-s, crucial for maintaining the pathwise integration accuracy in the stochastic regime, are provided. The method is developed within an adequately general framework to be readily implementable for large DOF mechanical systems. A host of numerical illustrations of the methods are undertaken for linear and non-linear, single- and multi-degree-of-freedom mechanical oscillators under additive and multiplicative white noise inputs. It may be noted that the lower order Newmark method has already been reported by the authors [16]. However, 
for the sake of completeness, the lower order method is again briefly covered, albeit within a more general framework.

\section{The methodology}

Since the stochastic Newmark method (SNM) is proposed to be developed in the context of engineering dynamics, a natural starting point would be to consider the following $n$-DOF dynamical system:

$$
\ddot{X}+C(X, \dot{X}) \dot{X}+K(X, \dot{X}) X=\sum_{r=1}^{q} G_{r}(X, \dot{X}, t) \dot{W}_{r}+F_{\mathrm{e}}(t),
$$

where $X=\left\{x^{(1)}, x^{(2)}, \ldots x^{(n)}\right\}^{\mathrm{T}} \in R^{2 n}, C(X, \dot{X}), K(X, \dot{X})$ are $n \times n$ (state-dependent for non-linear systems) damping and stiffness matrices, $\left\{G_{r}(X, \dot{X}, t)\right\}$ is the $r$ th element of a set of $n \times 1$ drift vectors, $\left\{W_{r}(t)\right\}$ constitutes a $q$-dimensional vector of independently evolving zero mean Wiener processes with $W_{r}(t)$, $r=1,2, \ldots q$ with $W_{r}(0)=0, E\left[\left|W_{r}(t)-W_{r}(s)\right|^{2}\right]=(t-s), t>s$ and $F_{\mathrm{e}}(t)=\left\{f_{\mathrm{e}}^{(j)}(t) \mid j=1,2, \ldots, n\right\}$ is the external (non-parametric) deterministic force vector. It may be noted that the white noise processes $\dot{W}_{r}(t)$ do not have any realizable sample paths with finite measure and hence the description of the dynamical system as in Eq. (1) is entirely formal. These second order equations may more appropriately be recast as the following system of $2 n$ first order equations in incremental form:

$$
\begin{aligned}
\mathrm{d} x_{1}^{(j)} & =x_{2}^{(j)} \mathrm{d} t, \\
\mathrm{~d} x_{2}^{(j)} & =a^{(j)}(X, \dot{X}, t) \mathrm{d} t+\sum_{r=1}^{q} \sigma_{r}^{(j)}(X, \dot{X}, t) \mathrm{d} W_{r}(t), \quad j=1,2, \ldots, n,
\end{aligned}
$$

where,

$$
\begin{aligned}
& a^{(j)}(X, \dot{X}, t)=-\sum_{k=1}^{n} C_{j k}(X, \dot{X}) \dot{x}^{(k)}-\sum_{k=1}^{n} K_{j k}(X, \dot{X}) x^{(k)}+f_{\mathrm{e}}^{(j)}(t), \\
& \sigma_{r}^{(j)}(X, \dot{X}, t)=G_{r}^{(j)}(X, \dot{X}, t) .
\end{aligned}
$$

In order to ensure sample existence and boundedness of the solution vectors $X_{1}=X=\left\{x_{1}^{(j)}\right\}$ and $X_{2}=\dot{X}=\left\{x_{2}^{(j)}\right\}, j=1,2, \ldots, n$, it is assumed that the drift and diffusion vectors, $a^{(j)}$ and $\sigma_{r}^{(j)}$ are measurable (with respect to $t \in R^{1}, X, \dot{X} \in R^{n}$ ), continuous and satisfy the Lipschitz (linear) growth bound:

$$
\left|a^{(j)}(\widehat{X}, t)-a^{(j)}(\widehat{Y}, t)\right|+\sum_{r=1}^{q}\left|\sigma_{r}^{(j)}(\widehat{X}, t)-\sigma_{r}^{(j)}(\widehat{Y}, t)\right| \leqslant Q|\widehat{X}-\widehat{Y}|, \quad \forall j \in[1, n],
$$

where $\widehat{X}, \widehat{Y} \in R^{2 n}, Q \in R^{+}$is a sufficiently large positive real number and $\widehat{X}=\left\{X^{\mathrm{T}} \dot{X}^{\mathrm{T}}\right\}^{\mathrm{T}}=\left\{X_{1}^{\mathrm{T}} X_{2}^{\mathrm{T}}\right\}^{\mathrm{T}}$. The norm $\|\cdot\|$ is the Euclidean norm. Let the initial conditions be m.s. bounded, i.e., $E\left|\widehat{X}\left(t_{0}\right)\right|^{2}<\infty$ and have certain growth bounds (not necessarily linear). Thus the sample continuity (w.p.1) of any realization of the (separable) vector flow $\phi\left(t, \omega, \widehat{X}\left(t_{0}\right)\right)$ for any $\omega \in \Omega(\Omega$ being the event space) is assured. Let the subset of the time axis over $[0, \tau]$ be ordered such that $0=t_{0}<t_{1}<t_{2}<\cdots<t_{i}<\cdots<t_{L}=\tau$ and $h_{i}=t_{i}-t_{i-1}$ where $i \in Z^{+}$. It is now required to replace the non-linear system of SDEs (1) by a suitably determined (stochastic) Newmark map over the $i$ th time interval $T_{i}=\left(t_{i-1}, t_{i}\right]$, given the initial condition vector $\widehat{X}\left(t_{i-1}\right) \triangleq \widehat{X}_{i-1}$. It is assumed that the response random variable $\widehat{X}\left(t_{i}\right) \triangleq \widehat{X}_{i}$ is $\mathbf{F}\left(t_{i}\right)$ measurable with $\mathbf{E}\left|\widehat{X}_{i}\right|^{2}<\infty$ and $\mathbf{F}\left(t_{i}\right)$ denoting the non-increasing family of $\sigma$-subalgebras. Further, for convenience of discussion, an uniform time step size $h_{i}=h \forall i$ is assumed in what follows.

Now, towards deriving the stochastic Newmark map over the $i$ th time interval, the first step is to consider Eq. (2) and expand each element of the vectors $X_{1}\left(t_{i-1}+h\right)=X\left(t_{i-1}+h\right)$ and $X_{2}\left(t_{i-1}+h\right)=$ $\dot{X}_{1}\left(t_{i-1}+h\right)$ in a stochastic Taylor expansion around $X_{1}\left(t_{i-1}\right)=X_{1, i-1}$ and $X_{2}\left(t_{i-1}\right)=X_{2, i-1}$ respectively. 
Either Ito or Stratonovich calculus may be used for this purpose (see [6] or [9] for a detailed derivation of Ito-Taylor and Ito-Stratonovich expansions). For purposes of a ready reference, a brief derivation of the Ito-Taylor expansion is provided in Appendix B. In the present study, the derivation of the map is performed following Ito's formula (see Appendix A), i.e., by using the Ito-Taylor expansion. An integral form of Ito's formula (see Appendix B) of any functional of response processes, as adapted specifically for Eq. (2), is stated below:

$$
\begin{aligned}
f\left(X_{1}(s), X_{2}(s), s\right)= & f\left(X_{1}\left(t_{i-1}\right), X_{2}\left(t_{i-1}\right), t_{i-1}\right)+\sum_{r=1}^{q} \int_{t_{i-1}}^{s} \Lambda_{r} f\left(X_{1}\left(s_{1}\right), X_{2}\left(s_{1}\right), s_{1}\right) \mathrm{d} W_{r}\left(s_{1}\right) \\
& +\int_{t_{i-1}}^{s} L f\left(X_{1}\left(s_{1}\right), X_{2}\left(s_{1}\right), s_{1}\right) \mathrm{d} s_{1},
\end{aligned}
$$

where $f$ is any sufficiently smooth (scalar or vector) function of its arguments, $s \geqslant t_{i-1}$ and the operators $\Lambda_{r}$ and $L$ are given by:

$$
\begin{aligned}
& \Lambda_{r}=\sum_{j=1}^{n} \sigma_{r}^{(j)} \frac{\partial f\left(X_{1}, X_{2}, t\right)}{\partial x_{2}^{(j)}} \\
& L=\frac{\partial f}{\partial t}+\sum_{j=1}^{n} x_{2}^{(j)} \frac{\partial f}{\partial x_{1}^{(j)}}+\sum_{j=1}^{n} a^{(j)} \frac{\partial f}{\partial x_{2}^{(j)}}+0.5 \sum_{r=1}^{q} \sum_{k=1}^{n} \sum_{l=1}^{n} \sigma_{r}^{(k)} \sigma_{r}^{(l)} \frac{\partial^{2} f}{\partial x_{2}^{(k)} \partial x_{2}^{(l)}} .
\end{aligned}
$$

\subsection{A lower order Newmark method}

Here the $j$ th element, $x_{1}^{(j)}\left(t_{i}=t_{i-1}+h\right)$, of the $n$-dimensional vector $X_{1}\left(t_{i}\right)$ is expanded over the semiclosed interval $\left(t_{i-1}, t_{i}\right]$ as:

$$
\begin{aligned}
x_{1}^{(j)}\left(t_{i}\right)= & x_{1}^{(j)}\left(t_{i-1}\right)+\int_{t_{i-1}}^{t_{i}} x_{2}^{(j)}(s) \mathrm{d} s=x_{1}^{(j)}\left(t_{i-1}\right)+\int_{t_{i-1}}^{t_{i}}\left\{x_{2}^{(j)}\left(t_{i-1}\right)+\int_{t_{i-1}}^{s} a^{(j)}\left(\widehat{X}\left(s_{1}\right), s_{1}\right) \mathrm{d} s_{1}\right. \\
& \left.+\sum_{r=1}^{q} \int_{t_{i-1}}^{s} \sigma_{r}^{(j)}\left(\widehat{X}\left(s_{1}\right), s_{1}\right) \mathrm{d} W_{r}\left(s_{1}\right)\right\} \mathrm{d} s .
\end{aligned}
$$

At this stage, Ito's formula (which forms the basis for stochastic Taylor expansions; Appendix A provides a brief account of Ito's formula) may be applied on the functions $\sigma_{r}^{(j)}$ and $a^{(j)}$ around $\left(\widehat{X}\left(t_{i-1}\right), t_{i-1}\right)$ to obtain the stochastic Taylor expansion:

$$
x_{1}^{(j)}\left(t_{i}\right)=x_{1}^{(j)}\left(t_{i-1}\right)+x_{2}^{(j)}\left(t_{i-1}\right) h+a^{(j)}\left(\widehat{X}\left(t_{i-1}\right), t_{i-1}\right) \frac{h^{2}}{2}+\sum_{r=1}^{q} \sigma_{r}^{(j)}\left(\widehat{X}\left(t_{i-1}\right), t_{i-1}\right) \int_{t_{i-1}}^{t_{i}} \int_{t_{i-1}}^{s} \mathrm{~d} W_{r}\left(s_{1}\right) \mathrm{d} s+\rho_{1}^{(j)},
$$

where the $j$ th remainder component, $\rho_{1}^{(j)}$, consists of the following multiple integrals:

$$
\begin{aligned}
\rho_{1}^{(j)}= & \sum_{r=1}^{q} \int_{t_{i-1}}^{t_{i}} \int_{t_{i-1}}^{s} \int_{t_{i-1}}^{s_{1}} \Lambda_{r} a^{(j)}\left(\widehat{X}\left(s_{2}\right), s_{2}\right) \mathrm{d} W_{r}\left(s_{2}\right) \mathrm{d} s_{1} \mathrm{~d} s+\int_{t_{i-1}}^{t_{i}} \int_{t_{i-1}}^{s} \int_{t_{i-1}}^{s_{1}} L a^{(j)}\left(\widehat{X}\left(s_{2}\right), s_{2}\right) \mathrm{d} s_{2} \mathrm{~d} s_{1} \mathrm{~d} s \\
& +\sum_{r=1}^{q} \sum_{l=1}^{q} \int_{t_{i-1}}^{t_{i}} \int_{t_{i-1}}^{s} \int_{t_{i-1}}^{s_{1}} \Lambda_{l} \sigma_{r}^{(j)}\left(\widehat{X}\left(s_{2}\right), s_{2}\right) \mathrm{d} W_{l}\left(s_{2}\right) \mathrm{d} W_{r}\left(s_{1}\right) \mathrm{d} s \\
& +\sum_{r=1}^{q} \int_{t_{i-1}}^{t_{i}} \int_{t_{i-1}}^{s} \int_{t_{i-1}}^{s_{1}} L \sigma_{r}^{(j)}\left(\widehat{X}\left(s_{2}\right), s_{2}\right) \mathrm{d} s_{2} \mathrm{~d} W_{r}\left(s_{1}\right) \mathrm{d} s .
\end{aligned}
$$


The above equations constitute a direct stochastic Taylor expansion for the displacement vector $X_{1}$. However, keeping in mind the deterministic Newmark technique, an implicitness is introduced in the expansion by using a non-unique real integration parameter $\alpha$ and writing Eq. (7a) as:

$$
\begin{aligned}
x_{1}^{(j)}\left(t_{i}\right)= & x_{1}^{(j)}\left(t_{i-1}\right)+x_{2}^{(j)}\left(t_{i-1}\right) h+\sum_{r=1}^{q} \sigma_{r}^{(j)}\left(\widehat{X}\left(t_{i-1}\right), t_{i-1}\right) \int_{t_{i-1}}^{t_{i}} \int_{t_{i-1}}^{s} \mathrm{~d} W_{r}\left(s_{1}\right) \mathrm{d} s+\alpha a^{(j)}\left(\widehat{X}\left(t_{i-1}\right), t_{i-1}\right) \frac{h^{2}}{2} \\
& +(1-\alpha) a^{(j)}\left(\widehat{X}\left(t_{i-1}\right), t_{i-1}\right) \frac{h^{2}}{2}+\rho_{1}^{(j)} .
\end{aligned}
$$

Now the fifth term on the RHS of the above equation is expressed in terms of $a^{(j)}\left(\widehat{X}\left(t_{i}\right), t_{i}\right)$ via a backward stochastic Taylor expansion as:

$$
a^{(j)}\left(\widehat{X}\left(t_{i-1}\right), t_{i-1}\right)=a^{(j)}\left(\widehat{X}\left(t_{i}\right), t_{i}\right)-\rho_{2}^{(j)},
$$

where the remainder $\rho_{2}^{(j)}$ is given by:

$$
\rho_{2}^{(j)}=\int_{t_{i-1}}^{t_{i}} L a^{(j)}(\widehat{X}(s), s) \mathrm{d} s+\sum_{r=1}^{q} \int_{t_{i-1}}^{t_{i}} \Lambda_{r} a^{(j)}(\widehat{X}(s), s) \mathrm{d} W_{r}(s) .
$$

Thus one has the following expression for $x_{1}^{(j)}\left(t_{i}\right) \triangleq x_{1, i}^{(j)}$ :

$$
\begin{aligned}
x_{1, i}^{(j)}= & x_{1, i-1}^{(j)}+x_{2, i-1}^{(j)} h+\sum_{r=1}^{q} \sigma_{r}^{(j)}\left(\widehat{X}_{i-1}, t_{i-1}\right) \int_{t_{i-1}}^{t_{i}} \int_{t_{i-1}}^{s} \mathrm{~d} W_{r}\left(s_{1}\right) \mathrm{d} s+\alpha a^{(j)}\left(\widehat{X}_{i-1}, t_{i-1}\right) \frac{h^{2}}{2} \\
& +(1-\alpha) a^{(j)}\left(\widehat{X}_{i}, t_{i}\right) \frac{h^{2}}{2}+R^{(j)}, \\
R^{(j)}= & -(1-\alpha) \rho_{2}^{(j)} \frac{h^{2}}{2}+\rho_{1}^{(j)} .
\end{aligned}
$$

The $j$ th velocity component $x_{2, i}^{(j)}$ is now implicitly expanded as:

$$
x_{2, i}^{(j)}=x_{2, i-1}^{(j)}+\sum_{r=1}^{q} \sigma_{r}^{(j)}\left(\widehat{X}_{i-1}, t_{i-1}\right) \int_{t_{i-1}}^{t_{i}} \mathrm{~d} W_{r}(s)+\beta a^{(j)}\left(\widehat{X}_{i-1}, t_{i-1}\right) h+(1-\beta) a^{(j)}\left(\widehat{X}_{i}, t_{i}\right) h+R_{1}^{(j)},
$$

where, the expression for the remainder is:

$$
\begin{aligned}
R_{1}^{(j)}= & \sum_{r=1}^{q} \int_{t_{i-1}}^{t_{i}} \mathrm{~d} W_{r}(s) \int_{t_{i-1}}^{s} \sum_{l=1}^{q} \Lambda_{l} \sigma_{r}^{(j)}\left(\widehat{X}\left(s_{1}\right), s_{1}\right) \mathrm{d} W_{l}\left(s_{1}\right) \\
& +\sum_{r=1}^{q} \int_{t_{i-1}}^{t_{i}} \mathrm{~d} W_{r}(s) \int_{t_{i-1}}^{s} L \sigma_{r}^{(j)}\left(\widehat{X}\left(s_{1}\right), s_{1}\right) \mathrm{d} s_{1}-(1-\beta) \rho_{2}^{(j)} h .
\end{aligned}
$$

In the RHS of the above expression, $\rho_{2}^{(j)}$ is given by Eq. (9b). Now a stochastic Newmark map for the $j$ th scalar displacement, $x_{1}^{(j)}$, is given by Eq. (10a) without the last remainder term, $R^{(j)}$, on the RHS. Similarly, a Newmark map for the $j$ th scalar velocity, $x_{2}^{(j)}$, is obtained via Eq. (11) without the remainder term $R_{1}^{(j)}$ on the RHS. These Newmark approximations to $\{\widehat{X}\}=\left\{\left\{x_{1}^{(j)}\right\}^{\mathrm{T}}\left\{x_{2}^{(j)}\right\}^{\mathrm{T}} \mid j=1,2, \ldots, n\right\}^{\mathrm{T}}$ will henceforth be denoted as $\{\tilde{X}\}=\left\{\left\{\tilde{x}_{1}^{(j)}\right\}^{\mathrm{T}},\left\{\tilde{x}_{2}^{(j)}\right\}^{\mathrm{T}} \mid j=1,2, \ldots, n\right\}^{\mathrm{T}}$.

\subsection{A higher order Newmark method}

As in the deterministic case, stochastic analogs of higher order Newmark methods are possible, at least theoretically. Such higher order methods may be derived by incorporating more terms in the associated 
stochastic Taylor expansions (i.e, by iterating the error terms in lower order Newmark method via an integral form of Ito's formula (see Appendix B for a general description of deriving the Ito-Taylor expansion). However, in sharp contrast with the lower order Newmark method, one has the daunting task of modelling MSI-s of third and higher levels for higher order Newmark methods. For notational simplicity, these multiple integrals will henceforth be denoted by:

$$
I_{j_{1}, j_{2}, \ldots, j_{k}}=\int_{t_{i-1}}^{t_{i}} \mathrm{~d} W_{j_{k}}(s) \int_{t_{i-1}}^{s} \mathrm{~d} W_{j_{k-1}}\left(s_{1}\right) \int_{t_{i-1}}^{s_{1}} \cdots \int_{t_{i-1}}^{s_{k-2}} \mathrm{~d} W_{j_{1}}\left(s_{k-1}\right),
$$

where the integers $j_{1}, j_{2}, \ldots, j_{k}$ take values in the set $\{0,1,2, \ldots, q\}$ and $I_{j_{1}, j_{2}, \ldots, j_{k}}$ may be considered as the $k$ th Ito multiple integral. Moreover, $\mathrm{d} W_{0}(s)$ is taken to indicate $\mathrm{d} s$. In most of the cases, these higher level stochastic integrals cannot be evaluated in closed form and hence need to be determined numerically. Even a numerical computation may sometimes become so computationally expensive as to render the higher order method practically useless. With this in mind, only one higher order Newmark scheme, which can be applied to an engineering system of arbitrarily high DOF and under any number of additive and/or multiplicative white noise processes, hopefully within an affordably extra computational cost, is presented in what follows.

Following the same stochastic expansion of Ito as in the lower order case and using the new symbol, as in Eq. (13), for multiple stochastic integrals, the following higher order implicit expansion for $x_{1, i}^{(j)}$ may be arrived at:

$$
\begin{aligned}
x_{1, i}^{(j)}= & x_{1, i-1}^{(j)}+x_{2, i-1}^{(j)} h+a^{(j)}\left(\widehat{X}_{i-1}, t_{i-1}\right) \frac{h^{2}}{2}+\sum_{r=1}^{q} \sigma_{r}^{(j)}\left(\widehat{X}_{i-1}, t_{i-1}\right) I_{r, 0}+\sum_{r=1}^{q} \sum_{l=1}^{q} \Lambda_{l} \sigma_{r}^{(j)}\left(\widehat{X}_{i-1}, t_{i-1}\right) I_{l, r, 0} \\
& +\sum_{r=1}^{q} \sum_{l=1}^{q} \sum_{u=1}^{q} \Lambda_{u} \Lambda_{l} \sigma_{r}^{(j)}\left(\widehat{X}_{i-1}, t_{i-1}\right) I_{u, l, r, 0}+\sum_{r=1}^{q} \Lambda_{r} a^{(j)}\left(\widehat{X}_{i-1}, t_{i-1}\right) I_{r, 0,0} \\
& +\sum_{r=1}^{q} L \sigma_{r}^{(j)}\left(\widehat{X}_{i-1}, t_{i-1}\right) I_{0, r, 0}+\left\{\alpha L a^{(j)}\left(\widehat{X}_{i-1}, t_{i-1}\right)+(1-\alpha) L a^{(j)}\left(\widehat{X}_{i}, t_{i}\right)\right\} \frac{h^{3}}{6}+R_{2}^{(j)},
\end{aligned}
$$

where the higher order remainder $R_{2}^{(j)}$ is given by:

$$
R_{2}^{(j)}=\rho_{3}^{(j)}-(1-\alpha) \rho_{4}^{(j)} \frac{h^{3}}{6} .
$$

The error components $\rho_{3}^{(j)}$ and $\rho_{4}^{(j)}$ are respectively given by:

$$
\begin{aligned}
\rho_{3}^{(j)}= & \sum_{r=1}^{q} \sum_{l=1}^{q} \int_{t_{i-1}}^{t_{i}} \int_{t_{i-1}}^{s} \int_{t_{i-1}}^{s_{1}} \int_{t_{i-1}}^{s_{2}} L \Lambda_{l} \sigma_{r}^{(j)}\left(\widehat{X}\left(s_{3}\right), s_{3}\right) \mathrm{d} s_{3} \mathrm{~d} W_{l}\left(s_{2}\right) \mathrm{d} W_{r}\left(s_{1}\right) \mathrm{d} s \\
& +\sum_{r=1}^{q} \sum_{l=1}^{q} \sum_{u=1}^{q} \int_{t_{i-1}}^{t_{i}} \int_{t_{i-1}}^{s} \int_{t_{i-1}}^{s_{1}} \int_{t_{i-1}}^{s_{2}} \int_{t_{i-1}}^{s_{3}} L \Lambda_{u} \Lambda_{l} \sigma_{r}^{(j)}\left(\widehat{X}\left(s_{4}\right), s_{4}\right) \mathrm{d} s_{4} \mathrm{~d} W_{u}\left(s_{3}\right) \mathrm{d} W_{l}\left(s_{2}\right) \mathrm{d} W_{r}\left(s_{1}\right) \mathrm{d} s \\
& +\sum_{r=1}^{q} \sum_{l=1}^{q} \sum_{u=1}^{q} \sum_{v=1}^{q} \int_{t_{i-1}}^{t_{i}} \int_{t_{i-1}}^{s} \int_{t_{i-1}}^{s_{1}} \int_{t_{i-1}}^{s_{2}} \int_{t_{i-1}}^{s_{3}} \Lambda_{v} \Lambda_{u} \Lambda_{l} \sigma_{r}^{(j)}\left(\widehat{X}\left(s_{4}\right), s_{4}\right) \mathrm{d} W_{v}\left(s_{4}\right) \mathrm{d} W_{u}\left(s_{3}\right) \mathrm{d} W_{l}\left(s_{2}\right) \mathrm{d} W_{r}\left(s_{1}\right) \mathrm{d} s \\
& +\sum_{r=1}^{q} \int_{t_{i-1}}^{t_{i}} \int_{t_{i-1}}^{s} \int_{t_{i-1}}^{s_{1}} \int_{t_{i-1}}^{s_{2}} L \Lambda_{r} a^{(j)}\left(\widehat{X}\left(s_{3}\right), s_{3}\right) \mathrm{d} s_{3} \mathrm{~d} W_{r}\left(s_{2}\right) \mathrm{d} s_{1} \mathrm{~d} s \\
& +\sum_{r=1}^{q} \sum_{l=1}^{q} \int_{t_{i-1}}^{t_{i}} \int_{t_{i-1}}^{s} \int_{t_{i-1}}^{s_{1}} \int_{t_{i-1}}^{s_{2}} \Lambda_{l} \Lambda_{r} a^{(j)}\left(\widehat{X}\left(s_{3}\right), s_{3}\right) \mathrm{d} W_{l}\left(s_{3}\right) \mathrm{d} W_{r}\left(s_{2}\right) \mathrm{d} s_{1} \mathrm{~d} s
\end{aligned}
$$




$$
\begin{aligned}
& +\sum_{r=1}^{q} \int_{t_{i-1}}^{t_{i}} \int_{t_{i-1}}^{s} \int_{t_{i-1}}^{s_{1}} \int_{t_{i-1}}^{s_{2}} L^{2} \sigma_{r}^{(j)}\left(\widehat{X}\left(s_{3}\right), s_{3}\right) \mathrm{d} s_{3} \mathrm{~d} s_{2} \mathrm{~d} W_{r}\left(s_{1}\right) \mathrm{d} s \\
& +\sum_{r=1}^{q} \sum_{l=1}^{q} \int_{t_{i-1}}^{t_{i}} \int_{t_{i-1}}^{s} \int_{t_{i-1}}^{s_{1}} \int_{t_{i-1}}^{s_{2}} \Lambda_{l} \sigma_{r}^{(j)}\left(\widehat{X}\left(s_{3}\right), s_{3}\right) \mathrm{d} W_{l}\left(s_{3}\right) \mathrm{d} s_{2} \mathrm{~d} W_{r}\left(s_{1}\right) \mathrm{d} s \\
& +\int_{t_{i-1}}^{t_{i}} \int_{t_{i-1}}^{s} \int_{t_{i-1}}^{s_{1}} \int_{t_{i-1}}^{s_{2}} L^{2} a^{(j)}\left(\widehat{X}\left(s_{3}\right), s_{3}\right) \mathrm{d} s_{3} \mathrm{~d} s_{2} \mathrm{~d} s_{1} \mathrm{~d} s \\
& +\sum_{r=1}^{q} \int_{t_{i-1}}^{t_{i}} \int_{t_{i-1}}^{s} \int_{t_{i-1}}^{s_{1}} \int_{t_{i-1}}^{s_{2}} \Lambda_{r} L a^{(j)}\left(\widehat{X}\left(s_{3}\right), s_{3}\right) \mathrm{d} W_{r}\left(s_{3}\right) \mathrm{d} s_{2} \mathrm{~d} s_{1} \mathrm{~d} s, \\
\rho_{4}^{(j)}= & \int_{t_{i-1}}^{t_{i}} L^{2} a^{(j)}(\widehat{X}(s), s) \mathrm{d} s+\sum_{r=1}^{q} \int_{t_{i-1}}^{t_{i}} \Lambda_{r} L a^{(j)}(\widehat{X}(s), s) \mathrm{d} W_{r}(s) .
\end{aligned}
$$

The higher order implicit expansion for the $j$ th velocity component, $x_{2, i}^{(j)}$, may similarly be derived as:

$$
\begin{aligned}
x_{2, i}^{(j)}= & x_{2, i-1}^{(j)}+\sum_{r=1}^{q} \sigma_{r}^{(j)}\left(\widehat{X}_{i-1}, t_{i-1}\right) I_{r}+\sum_{r=1}^{q} \sum_{l=1}^{q} \Lambda_{l} \sigma_{r}^{(j)}\left(\widehat{X}_{i-1}, t_{i-1}\right) I_{l, r}+\sum_{r=1}^{q} L \sigma_{r}^{(j)}\left(\widehat{X}_{i-1}, t_{i-1}\right) I_{0, r} \\
& +a^{(j)}\left(\widehat{X}_{i-1}, t_{i-1}\right) h+\sum_{r=1}^{q} \Lambda_{r} a^{(j)}\left(\widehat{X}_{i-1}, t_{i-1}\right) I_{r, 0}+\sum_{r=1}^{q} \sum_{l=1}^{q} \sum_{u=1}^{q} \Lambda_{u} \Lambda_{l} \sigma_{r}^{(j)}\left(\widehat{X}_{i-1}, t_{i-1}\right) I_{u, l, r} \\
& +\left\{\beta L a^{(j)}\left(\widehat{X}_{i-1}, t_{i-1}\right)+(1-\beta) L a^{(j)}\left(\widehat{X}_{i}, t_{i}\right)\right\} \frac{h^{2}}{2}+R_{3}^{(j)} .
\end{aligned}
$$

The $j$ th error component, $R_{3}^{(j)}$, corresponding to the velocity component, $x_{2}^{(j)}$, may be written as:

$$
R_{3}^{(j)}=\rho_{5}^{(j)}-(1-\beta) \rho_{4}^{(j)} \frac{h^{2}}{2}
$$

where,

$$
\begin{aligned}
\rho_{5}^{(j)}= & \sum_{r=1}^{q} \sum_{l=1}^{q} \sum_{u=1}^{q} \sum_{v=1}^{q} \int_{t_{i-1}}^{t_{i}} \int_{t_{i-1}}^{s} \int_{t_{i-1}}^{s_{1}} \int_{t_{i-1}}^{s_{2}} \Lambda_{v} \Lambda_{u} \Lambda_{l} \sigma_{r}^{(j)}\left(\widehat{X}\left(s_{3}\right), s_{3}\right) \mathrm{d} W_{v}\left(s_{3}\right) \mathrm{d} W_{u}\left(s_{2}\right) \mathrm{d} W_{l}\left(s_{1}\right) \mathrm{d} W_{r}(s) \\
& +\sum_{r=1}^{q} \sum_{l=1}^{q} \sum_{u=1}^{q} \int_{t_{i-1}}^{t_{i}} \int_{t_{i-1}}^{s} \int_{t_{i-1}}^{s_{1}} \int_{t_{i-1}}^{s_{2}} L \Lambda_{u} \Lambda_{l} \sigma_{r}^{(j)}\left(\widehat{X}\left(s_{3}\right), s_{3}\right) \mathrm{d} s_{3} \mathrm{~d} W_{u}\left(s_{2}\right) \mathrm{d} W_{l}\left(s_{1}\right) \mathrm{d} W_{r}(s) \\
& +\sum_{r=1}^{q} \sum_{l=1}^{q} \int_{t_{i-1}}^{t_{i}} \int_{t_{i-1}}^{s} \int_{t_{i-1}}^{s_{1}} \Lambda_{l} L \sigma_{r}^{(j)}\left(\widehat{X}\left(s_{2}\right), s_{2}\right) \mathrm{d} W_{l}\left(s_{2}\right) \mathrm{d} s_{1} \mathrm{~d} W_{r}(s) \\
& +\sum_{r=1}^{q} \int_{t_{i-1}}^{t_{i}} \int_{t_{i-1}}^{s} \int_{t_{i-1}}^{s_{1}} L^{2} \sigma_{r}^{(j)}\left(\widehat{X}\left(s_{2}\right), s_{2}\right) \mathrm{d} s_{2} \mathrm{~d} s_{1} \mathrm{~d} W_{r}(s) \\
& +\sum_{r=1}^{r} \sum_{l=1}^{q} \int_{t_{i-1}}^{t_{i}} \int_{t_{i-1}}^{s} \int_{t_{i-1}}^{s_{1}} \Lambda_{l} \Lambda_{r} a^{(j)}\left(\widehat{X}\left(s_{2}\right), s_{2}\right) \mathrm{d} W_{l}\left(s_{2}\right) \mathrm{d} W_{r}\left(s_{1}\right) \mathrm{d} s \\
& +\sum_{r=1}^{q} \int_{t_{i-1}}^{t_{i}} \int_{t_{i-1}}^{s} \int_{t_{i-1}}^{s_{1}} L \Lambda_{r} a^{(j)}\left(\widehat{X}\left(s_{2}\right), s_{2}\right) \mathrm{d} s_{2} \mathrm{~d} W_{r}\left(s_{1}\right) \mathrm{d} s \\
& +\sum_{r=1}^{q} \int_{t_{i-1}}^{t_{i}} \int_{t_{i-1}}^{s} \int_{t_{i-1}}^{s_{1}} \Lambda_{r} L a^{(j)}\left(\widehat{X}\left(s_{2}\right), s_{2}\right) \mathrm{d} W_{r}\left(s_{2}\right) \mathrm{d} s_{1} \mathrm{~d} s \\
& +\int_{t_{i-1}}^{t_{i}} \int_{t_{i-1}}^{s} \int_{t_{i-1}}^{s_{1}} L^{2} a^{(j)}\left(\widehat{X}\left(s_{2}\right), s_{2}\right) \mathrm{d} s_{2} \mathrm{~d} s_{1} \mathrm{~d} s
\end{aligned}
$$


and $\rho_{4}^{(j)}$ is given by Eq. (16b). Obviously, the higher order stochastic Newmark map for the $j$ th displacement and velocity components, $x_{1}^{(j)}$ and $x_{2}^{(j)}$, are respectively given via Eqs. (14) and (17) without the remainder terms.

\subsection{Error estimates}

Since the sample path, $\widehat{X}(t)$ traced by the given SDE is, in general, different from the approximated Newmark solution, $\widetilde{X}$, an instantaneous error at $t=t_{i}$ may be defined as the $2 n$-dimensional vector $E_{i}=\left\{\left\{E_{1, i}^{(j)}\right\}^{\mathrm{T}},\left\{E_{2, i}^{(j)}\right\}^{\mathrm{T}}\right\}^{\mathrm{T}}=\left\{\left\{\left(x_{1, i}^{(j)}-\tilde{x}_{1, i}^{(j)}\right)\right\}^{\mathrm{T}},\left\{\left(x_{2, i}^{(j)}-\tilde{x}_{2, i}^{(j)}\right)\right\}^{\mathrm{T}}\right\}^{\mathrm{T}}$ where $j=1, \ldots, n$, and the instantaneous Euclidean error norm is denoted as $e_{i}=\left|\widehat{X}_{i}-\widetilde{X}_{i}\right|$. This error vector is treated as a set of conditional random variables such that the local initial condition, $\widehat{X}_{i-1}$, is deterministic and that $\widehat{X}_{i-1}=\widetilde{X}_{i-1}$. In what follows, let $c_{\mathrm{m}}$ and $c_{\mathrm{s}}$ respectively denote the orders of the mean and mean square of this conditional (local) error with respect to the chosen time step size, $h=t_{i}-t_{i-1}$. In other words, one has the following bounds:

$$
\begin{aligned}
& \left\|\mathbf{E}\left(\widehat{X}_{i}-\widetilde{X}_{i}\right)\right\| \leqslant Q\left(1+\left\|\widehat{X}_{i-1}\right\|^{2}\right) h^{c_{\mathrm{m}}}, \\
& {\left[\mathbf{E}\left\|\widehat{X}_{i}-\widetilde{X}_{i}\right\|^{2}\right]^{\frac{1}{2}} \leqslant Q\left(1+\left\|\widehat{X}_{i-1}\right\|^{2}\right) h^{c_{\mathrm{s}}} .}
\end{aligned}
$$

Also let $c_{\mathrm{s}} 1 / 2$ and $c_{\mathrm{m}} c_{\mathrm{s}}+1 / 2$. Then, one has the following bound on the global error:

$$
\left[\mathbf{E}\left\|\widehat{X}_{i}-\widetilde{X}_{i}\right\|^{2}\right]^{\frac{1}{2}} \leqslant Q\left(1+\left\|\widehat{X}_{0}\right\|^{2}\right) h^{c_{\mathrm{s}}-\frac{1}{2}} .
$$

This implies that the global order of accuracy of the method, constructed using a one-step approximation, is $c_{\mathrm{g}}=c_{\mathrm{s}}-1 / 2$. The monograph by Milstein [9, pp. 12-17] is referred for a step-by-step proof of this observation.

\subsubsection{Error in lower order Newmark method}

From the remainder Eqs. (7b), (9b) and (12), it is clear that for evaluating the mean and mean-square error orders, it is necessary to determine the first two statistical moments of the multiple Ito integrals, $I_{r}$ and $I_{r, 0}$. At this stage, the following proposition becomes quite relevant.

Proposition 1. One has $\mathbf{E}\left(I_{j_{1}, j_{2}, \ldots, j_{k}}\right)=0$ if there exists at least one $j_{l} \neq 0, l=1,2, \ldots, k$. On the other hand, $\mathbf{E}\left(I_{j_{1}, j_{2}, \ldots, j_{k}}\right)=\mathrm{O}\left(h^{k}\right)$ if $j_{l}=0 \forall l \in[0, k]$. Moreover,

$$
\begin{aligned}
& {\left[\mathbf{E}\left(I_{j_{1}, j_{2}, \ldots, j_{k}}\right)^{2}\right]^{\frac{1}{2}}=\mathrm{O}\left(h^{w}\right),} \\
& \text { where } \quad w=\sum_{l=1}^{k}\left(2-\bar{j}_{l}\right) / 2, \bar{j}_{l}=1 \quad \text { if } j_{l} \neq 0, \quad \text { else } \bar{j}_{l}=0 .
\end{aligned}
$$

Proof of the first part of the above proposition regarding the mean is quite straightforward. For the second part, involving Eq. (17), reference is made to the monographs by Milstein [9] or Kloeden and Platen [7].

Now taking expectation of the remainder term $R^{(j)}$ associated with the $j$ th displacement component $x_{1, i}^{(j)}$ (Eqs. (10a) and (10b)), one can readily see via Proposition 1 and the inequality (13) that:

$$
\left|\mathbf{E} R^{(j)}\right| \leqslant Q\left(1+\left\|\widehat{X}_{i-1}\right\|^{2}\right) h^{3} .
$$

Similarly, using Eq. (17) and the inequality (14), it follows that:

$$
\left|\mathbf{E}\left(R^{(j)}\right)^{2}\right|^{\frac{1}{2}} \leqslant Q\left(1+\left\|\widehat{X}_{i-1}\right\|^{2}\right)^{\frac{1}{2}} h^{2} .
$$


Thus, for the proposed stochastic Newmark scheme as applied to displacements, one has $\left(c_{\mathrm{m}}\right)_{x}=3$, $\left(c_{\mathrm{s}}\right)_{x}=2\left(>\frac{1}{2}\right)$, and thus the inequality $\left(c_{\mathrm{m}}\right)_{x} \geqslant\left(c_{\mathrm{s}}\right)_{x}+1 / 2$ is satisfied. Hence the global error order for computing the displacement vector is given by $\left(c_{\mathrm{g}}\right)_{x}=1.5$. One can also apply the above arguments for the velocity equation (11) and the associated remainder equation (12) to obtain $\left(c_{\mathrm{m}}\right)_{\dot{x}}=2,\left(c_{\mathrm{s}}\right)_{\dot{x}}=1(>1 / 2)$. Thus the inequality $\left(c_{\mathrm{m}}\right)_{\dot{x}} \geqslant\left(c_{\mathrm{s}}\right)_{\dot{x}}+1 / 2$ is again satisfied to yield $\left(c_{\mathrm{g}}\right)_{\dot{x}}=1 / 2$.

It is of interest to note here that the implicit stochastic Taylor expansion of Eq. (10a) used to obtain the Newmark approximation, $\tilde{x}_{1, i}^{(j)}$, to the exact displacement component, $x_{1, i}^{(j)}$, is not complete in $\mathrm{O}\left(h^{2}\right)$. This may be verified form the expression of the remainder, $R^{(j)}$, (Eq. (10b) along with Eq. (7b)) which contains a multiple Ito-integral $I_{l, r, 0}=\mathrm{O}\left(h^{2}\right), l, r \neq 0$. Since evaluation of multiple integrals involving more than one integration of Wiener increments is a computationally expensive affair, especially for large DOF engineering problems, the $\mathrm{O}\left(h^{2}\right)$ term involving $I_{l, r, 0}$ has not been accounted for in the lower order version of Newmark displacement map. If, in addition, the other easily-evaluatable $\mathrm{O}\left(h^{2}\right)$ term, $u_{1}=\alpha a^{(j)}\left(\widehat{X}_{i-1}, t_{i-1}\right) \frac{h^{2}}{2}+$ $(1-\alpha) a^{(j)}\left(\widehat{X}_{i}, t_{i}\right) \frac{h^{2}}{2}$, is also left out of the expansion, then from Proposition 1 one would have $\left(c_{\mathrm{m}}\right)_{x}=2$, $\left(c_{\mathrm{s}}\right)_{x}=2$. Since the inequality $\left(c_{\mathrm{m}}\right)_{x} \geqslant\left(c_{\mathrm{s}}\right)_{x}+0.5$ is no longer satisfied, one must take $\left(c_{\mathrm{s}}\right)_{x}=1.5$ for evaluating the global error order $\left(c_{\mathrm{g}}\right)_{x}[6]$. Thus one finally has $\left(c_{\mathrm{g}}\right)_{x}=1.5-0.5=1.0$. Hence it is observed that even though the presently adopted Newmark expansion is not complete in $\mathrm{O}\left(h^{2}\right)$, retaining the term $u_{1}$ leads to an increase in $\left(c_{\mathrm{g}}\right)_{x}$ by 0.5 . Now, consider the other possibility of retaining the term involving the $\mathrm{O}\left(h^{2}\right)$ integral $I_{l, r, 0}$ in the expansion instead of the term $u_{1}$. In such a case, one readily obtains, again via Proposition $1,\left(c_{\mathrm{m}}\right)_{x}=2,\left(c_{\mathrm{s}}\right)_{x}=2$. Thus, one again gets $\left(c_{\mathrm{g}}\right)_{x}=1.0$, i.e., 0.5 less in order than is achieved by retaining $u_{1}$ instead of the term involving $I_{l, r, 0}$. However, if both these $\mathrm{O}\left(h^{2}\right)$ terms are included, then $\left(c_{\mathrm{m}}\right)_{x}=3.0$, $\left(c_{\mathrm{s}}\right)_{x}=2.5$, and hence $\left(c_{\mathrm{g}}\right)_{x}=2.0$, i.e., 0.5 more in order than the presently adopted scheme at the cost of an enhanced computational effort, which sharply increases with an increase in the system DOF. Similar arguments as above may be used to justify the inclusion of the $\mathrm{O}(h)$ term, $u_{2}=\beta a^{(j)}\left(\widehat{X}_{i-1}, t_{i-1}\right) h+\beta a^{(j)}\left(\widehat{X}_{i}, t_{i}\right) h$, and not the term involving $\mathrm{O}(h)$ multiple Ito integral $I_{l, r}(l, r \neq 0)$. Thus, it may generally be noted that computations of error order along with the retention of appropriate terms in the stochastic Newmark method considerably differ from those for its well known deterministic counterparts.

\subsubsection{Error in higher order Newmark method}

Determination of local and global error orders for displacement and velocity components follows the same steps as detailed in Section 3.1. Thus taking the expectation of the error component $R_{2}^{(j)}$, as in Eq. (15a), corresponding to the displacement scalar $x_{1}^{(j)}$, it is readily seen that:

$$
\left|\mathbf{E} R_{2}^{(j)}\right| \leqslant Q\left(1+\left\|\widehat{X}_{i-1}\right\|^{2}\right) h^{4} .
$$

Moreover, using proposition (1), one also has:

$$
\left|\mathbf{E}\left(R_{2}^{(j)}\right)^{2}\right|^{\frac{1}{2}} \leqslant Q\left(1+\left\|\widehat{X}_{i-1}\right\|^{2}\right)^{\frac{1}{2}} h^{3} .
$$

Hence the local and global error orders for the (higher order) Newmark displacement vector, $\widetilde{X}_{1}$, are respectively $\mathrm{O}\left(h^{3}\right)$ and $\mathrm{O}\left(h^{2.5}\right)$. Similarly it may be shown using Eq. (18a) that the local and global error orders for the (higher order) Newmark velocity vector, $\widetilde{X}_{2}$, are respectively $\mathrm{O}\left(h^{2}\right)$ and $\mathrm{O}\left(h^{1.5}\right)$. It is thus apparent that the higher order Newmark scheme yields response approximations which are consistently one integral order higher than those obtained using the lower order Newmark scheme. However, this observation is true provided one can obtain the associated MSI-s sufficiently accurately.

\subsection{Determining the multiple stochastic integrals (MSI-s)}

Accurate computation of these integrals constitutes a crucial factor in maintaining the error orders as obtained in Section 3. First consider the lower order Newmark scheme, wherein one needs to compute only 
two kinds of multiple integrals, viz., $I_{r}$ and $I_{r, 0}, r=1,2, \ldots, q$. Fortunately, given the associated Wiener increments, both these integrals may be computed exactly. For the first level integral, $I_{r}$, one has $I_{r}=W_{r}\left(t_{i}\right)-W_{r}\left(t_{i-1}\right) \triangleq \Delta_{i} W_{r}$. To model $I_{r, 0}$, it is first noted (via integration by parts) that:

$$
I_{r, 0}=\int_{t_{i-1}}^{t_{i}} \int_{t_{i-1}}^{s} \mathrm{~d} W_{r}\left(s_{1}\right) \mathrm{d} s=h \Delta_{i} W_{r}-I_{0, r} .
$$

Moreover, using the Ito definition of a stochastic integral, one has:

$$
\mathbf{E}\left(I_{0, r}\right)=0 \quad \text { and } \quad \mathbf{E}\left(I_{0, r}\right)^{2}=\frac{h^{3}}{3} .
$$

It is therefore possible to model $I_{r, 0}$ as the random variable $v_{2}=h \Delta_{i} W_{r}-v_{1}$, where the zero-mean random variable $v_{1}$ has the normal distribution $N\left(0, \sqrt{h^{3} / 3}\right)$.

For the higher order Newmark scheme, the additional multiple integrals to be modeled are $I_{r, l}, I_{r, l, 0}$, $I_{r, 0,0}, I_{0, r, 0}, I_{r, l, u}$ and $I_{r, l, u, 0}$ where $r, l, u=1,2, \ldots, q$. In order to consistently generate these random variables numerically within a computer program, the following scaling is first effected.

$$
g_{r}(\theta)=\frac{W_{r}(\theta h)}{\sqrt{h}} \quad \text { with } \quad W_{r}(0)=0, \quad 0 \leqslant \theta \leqslant 1 .
$$

Obviously, $g_{r}(\theta), r=1,2, \ldots, q$ are standard Wiener processes with unit variance. Let the scaled $k$ th stochastic integral in terms of increments of new Wiener processes, $g_{r}(\theta)$ be denoted as:

$$
\bar{I}_{j_{1}, j_{2}, \ldots, j_{k}}=\int_{0}^{1} \int_{0}^{\theta} \ldots \int_{0}^{\theta_{k-2}} \mathrm{~d} g_{j_{1}}\left(\theta_{k-1}\right) \mathrm{d} g_{j_{2}}\left(\theta_{k-2}\right) \ldots \mathrm{d} g_{j_{k}}(\theta),
$$

where it is implied, as before, that $\mathrm{d} g_{0}(\theta)=\mathrm{d} \theta$. One thus has the following set of relations between the scaled and original multiple integrals:

$$
I_{r}=h^{\frac{1}{2}} \bar{I}_{r} ; \quad I_{r, 0}=h^{\frac{3}{2}} \bar{I}_{r, 0} ; \quad I_{0, r}=h^{\frac{3}{2}} \bar{I}_{0, r} ; \quad I_{r, l, 0}=h^{2} \bar{I}_{r, l, 0} ; \quad I_{r, l, u, 0}=h^{\frac{5}{2}} \bar{I}_{r, l, u, 0} ; \ldots \text { etc. }
$$

for all $r, l, u=1,2, \ldots, q$. Since the present objective is to generate the third or higher level integrals approximately via a numerical scheme, the following proposition becomes quite useful (Milstein [9]).

Proposition 2. Let a numerical scheme with an order of accuracy $m$, generates the following one-step approximation:

$$
\widetilde{X}_{i+1}=\widehat{X}_{i}+A\left(t_{i}, X_{i}, h, \Delta W_{r}(s) \mid t_{i} \leqslant s \leqslant t_{i}+h=t_{i+1} \quad \text { and } \quad r=1,2, \ldots, q\right) .
$$

Suppose that the vector function A contains terms of the form $P\left(t_{i}, X_{i}\right) \zeta\left(\Delta W_{r}(s)\right)$, where $\zeta($.$) is a known func-$ tions of its random arguments. If an approximate numerical method is used to generate the function $\zeta$ such that $\zeta=\vartheta+\delta$ (where $\vartheta$ is the approximate value and $\delta$ is the remainder), then the order of accuracy, $m$, of the original method remains unaltered if the following inequalities hold.

$$
|\mathbf{E} \delta| \leqslant Q h^{m+1} \quad \text { and } \quad\left[\mathbf{E} \delta^{2}\right]^{\frac{1}{2}} \leqslant Q h^{m+\frac{1}{2}} .
$$

\subsubsection{The case of a single white noise}

This particular case, involving only one Wiener process $W_{1}(t)$ is simpler to implement and is therefore dealt with first. It has been shown [9] that recursively using an expansion of the two-parameter Hermite $n$ polynomial, $H_{n}\left(\lambda s, \gamma W_{1}(s)\right)$, followed by equating the like powers of $\lambda$ and $\gamma$, one can arrive at the following exact expressions of the still undetermined multiple integrals: 


$$
\begin{aligned}
& I_{11}(h)=\frac{W_{1}^{2}(h)-h}{2} ; \quad I_{110}(h)=\frac{1}{2} \int_{0}^{h} W_{1}^{2}(s) \mathrm{d} s-\frac{h^{2}}{4}, \\
& I_{100}(h)=h I_{10}-\int_{0}^{h} s W_{1}(s) \mathrm{d} s ; \quad I_{010}(h)=2 \int_{0}^{h} s W_{1}(s) \mathrm{d} s-h I_{10}, \\
& I_{111}(h)=\frac{W_{1}^{3}(h)}{6}-\frac{1}{2} h W_{1}(h) ; \quad I_{1110}(h)=\frac{1}{6} \int_{0}^{h} W_{1}^{3}(s) \mathrm{d} s-\frac{1}{2} \int_{0}^{h} s W_{1}(s) \mathrm{d} s .
\end{aligned}
$$

Thus for an implementation of the higher order Newmark scheme, it suffices to approximately model the following basic multiple stochastic integrals: $A_{1}=\int_{0}^{h} W_{1}^{2}(s) \mathrm{d} s ; A_{2}=\int_{0}^{h} s W_{1}(s) \mathrm{d} s$; and $A_{3}=\int_{0}^{h} W_{1}^{3}(s) \mathrm{d} s$. Expressed in terms of the standard Wiener process, $g_{1}(\theta)=W_{1}(\theta h) / \sqrt{h}, \theta=s / h \in[0,1]$, these integrals take the form:

$$
\begin{aligned}
& A_{1}=h^{2} \int_{0}^{1} g_{1}^{2}(\theta) \mathrm{d} \theta=h^{2} \bar{A}_{1}, \\
& A_{2}=h^{5 / 2} \int_{0}^{1} \theta g_{1}(\theta) \mathrm{d} \theta=h^{5 / 2} \bar{A}_{2}, \\
& A_{3}=h^{5 / 2} \int_{0}^{1} g_{1}^{3}(\theta) \mathrm{d} \theta=h^{5 / 2} \bar{A}_{3} .
\end{aligned}
$$

Now the following four SDEs may be solved over $\theta \in[0,1]$ to determine these integrals:

$$
\mathrm{d} \bar{A}_{0}=\mathrm{d} g_{1}(\theta) ; \quad \mathrm{d} \bar{A}_{1}=\bar{A}_{0}^{2} \mathrm{~d} \theta ; \quad \mathrm{d} \bar{A}_{2}=\theta \bar{A}_{0}(\theta) \mathrm{d} \theta ; \quad \mathrm{d} \bar{A}_{3}=\bar{A}_{0}^{3}(\theta) \mathrm{d} \theta .
$$

The above equations are subject to initial conditions $\bar{A}_{k}(0)=0 ; k=0,1,2,3$. Moreover, the approximate numerical technique and the time step size, $h_{1}$, to be used to solve for these equations have to be so chosen as to satisfy the requirements of Proposition 2. In order to maintain local error orders $\mathrm{O}\left(h^{3}\right)$ and $\mathrm{O}\left(h^{2}\right)$ respectively for displacement and velocity components in the higher order Newmark scheme, the following inequalities need to be satisfied (via Proposition 2):

$$
\begin{aligned}
& \mathbf{E}\left[\left(\bar{A}_{1}(\theta=1)-\bar{A}_{1 N}(\theta=1)\right)^{2}\right]^{\frac{1}{2}} \leqslant \mathrm{O}\left(h^{1.5}\right), \\
& \mathbf{E}\left[\left(\bar{A}_{2}(\theta=1)-\bar{A}_{2 N}(\theta=1)\right)^{2}\right]^{\frac{1}{2}} \leqslant \mathrm{O}(h), \\
& \mathbf{E}\left[\left(\bar{A}_{3}(\theta=1)-\bar{A}_{3 N}(\theta=1)\right)^{2}\right]^{\frac{1}{2}} \leqslant \mathrm{O}(h),
\end{aligned}
$$

where, $\bar{A}_{1 N}, \bar{A}_{2 N}$ and $\bar{A}_{3 N}$ are numerical approximations to $\bar{A}_{1}, \bar{A}_{2}$ and $\bar{A}_{3}$ respectively and the above set of SDEs are subject to initial conditions $\bar{A}_{k N}(0)=0$, for $k=1,2,3$. It is noted that no such restrictions are put on the variable $\bar{A}_{0}$, since it can be modeled exactly using $\bar{A}_{0, j}=\bar{A}_{0, j-1}+\Delta_{j} g_{1}\left(h_{1}\right)$, where $\Delta_{j} g_{1}\left(h_{1}\right)$ is an $N\left(0, \sqrt{h_{1}}\right)$ random variable. At this stage, suppose that a stochastic Heun scheme (SHS) of global order $\mathrm{O}(h)$ is used to approximately obtain $\bar{A}_{1}(1), \bar{A}_{2}(1)$ and $\bar{A}_{3}(1)$ via the map:

$$
\begin{aligned}
& \bar{A}_{1 N, i}=\bar{A}_{1 N, i-1}+0.5\left(\bar{A}_{0, i-1}^{2}+\bar{A}_{0, i}^{2}\right) h_{1}, \\
& \bar{A}_{2 N, i}=\bar{A}_{2 N, i-1}+0.5\left(\theta_{i-1} \bar{A}_{0, i-1}+\theta_{i} \bar{A}_{0, i}\right) h_{1}, \\
& \bar{A}_{3 N, i}=\bar{A}_{3 N, i-1}+0.5\left(\bar{A}_{0, i-1}^{3}+\bar{A}_{0, i}^{3}\right) h_{1} .
\end{aligned}
$$

Now in order to satisfy the first of inequalities (37), one must have $h_{1}=h^{1.5}$, so that a smaller time step size, $h_{1}$, is warranted for accurately obtaining the required integrals. For instance, if a step size $h=0.01$ is used for integrating the original system of equations, then a corresponding step size $h_{1}=0.001$ needs to be chosen for approximately obtaining the multiple integrals over each interval. In other words, about 1000 random variables have to be generated over each time interval of $h=0.01$. On the other hand, if one 
forcibly chooses $h_{1}=h$ to economize on the computational time, the local error order for displacement components reduce to $\mathrm{O}\left(h^{2.5}\right)$, while the local error order of $\mathrm{O}\left(h^{2}\right)$ for velocity components remains unaffected. In fact, depending on specific forms of the governing differential equations, there may not be any need to model many such multiple integrals and thus one may still achieve the desired order of $\mathrm{O}\left(h^{3}\right)$ for the displacement components with $h_{1}=h$. Take, for instance, the common case where the multiplicative noise coefficients $\sigma_{r}^{(j)}\left(X_{1}, X_{2}, t\right)$ are not functions of the velocity vector, $X_{2}$. In such cases, $\Lambda_{l} \sigma_{r}^{(j)}=$ $\Lambda_{l} \Lambda_{u} \sigma_{r}^{(j)}=0$ and hence, as seen from the higher order Newmark displacement expansion of Eq. (14), all the terms involving $I_{l, r, 0}$ and $I_{l, r, u, 0}$ identically vanish, thereby ensuring a local error order of $\mathrm{O}\left(h^{3}\right)$ for the displacement components with $h_{1}=h$.

\subsubsection{The case of multiple white noise inputs}

A procedure similar to the case of a single white noise input may be adopted in this case too. The only difference in this case is that a simplified recursive relationship between the multiple integrals based on Hermite expansions is not possible here. Thus all the multiple integrals of third and higher levels have to be determined by constructing a set of simple SDE-s and solving the latter numerically. As in Section 2.4.1, these SDE-s are formed in terms of the increments of a set of standard (scaled) Wiener processes $g_{r}(\theta)=\frac{W_{r}(\theta h)}{\sqrt{h}}$, so that $0 \leqslant \theta \leqslant 1, r=1,2, \ldots, q(q \geqslant 2)$. With a time step size $h_{1}$, the first level scaled integrals, $\bar{I}_{r}(1)$, may be exactly generated using the map $\left(\bar{I}_{r}\right)_{j}=\left(\bar{I}_{r}\right)_{j-1}+\Delta_{j} g_{r}\left(h_{1}\right)$ with $\bar{I}_{r}(0)=\left(\bar{I}_{r}\right)_{0}=0$. Moreover, Eqs. (27) and (28) may be used to exactly obtain the second level integrals $\bar{I}_{r, 0}$ and $\bar{I}_{0, r}$. Next, one has the following set of SDE-s for the approximate evaluations of other scaled multiple integrals over $[0,1]$ with a step size $h_{1}$ :

$$
\begin{aligned}
& \mathrm{d} \bar{I}_{l, r}=\bar{I}_{l} \mathrm{~d} g_{r}(\theta) ; \quad \mathrm{d} \bar{I}_{l, r, 0}=\bar{I}_{l, r} \mathrm{~d} \theta ; \quad \mathrm{d} \bar{I}_{r, 0,0}=\bar{I}_{r, 0} \mathrm{~d} \theta, \\
& \mathrm{d} \bar{I}_{0, r, 0}=\bar{I}_{0, r} \mathrm{~d} \theta ; \quad \mathrm{d} \bar{I}_{u, l, r}=\bar{I}_{u, l} \mathrm{~d} g_{r}(\theta) ; \quad \mathrm{d} \bar{I}_{u, l, r, 0}=\bar{I}_{u, l, r} \mathrm{~d} \theta
\end{aligned}
$$

subject to initial conditions $\bar{I}_{l, r}(0)=0 ; \bar{I}_{l, r, 0}(0)=0 ; \ldots \bar{I}_{u, l, r, 0}(0)=0$. The following general relation between the scaled and original $k$ th level multiple integrals may be readily noted:

$$
I_{j_{1}, j_{2}, \ldots, j_{k}}\left(h,\left\{W_{r}(h) \mid r=1, \ldots, q\right\}\right)=h^{\sum_{m=1}^{k}\left(2-\bar{j}_{m}\right) / 2} \bar{I}_{j_{1}, j_{2}, \ldots, j_{k}}\left(1,\left\{g_{r}(1) \mid r=1, \ldots, q\right\}\right),
$$

where $\bar{j}_{m}=0$ if $j_{m}=0$ else $\bar{j}_{m}=1$. Now suppose that a Heun scheme be used to determine the scaled multiple integrals as:

$$
\begin{aligned}
& \left(\bar{I}_{l, r}\right)_{j}=\left(\bar{I}_{l, r}\right)_{j-1}+0.5\left(\left(\bar{I}_{l}\right)_{j-1}+\left(\bar{I}_{l}\right)_{j}\right) \Delta_{j} g_{r}\left(h_{1}\right), \\
& \left(\bar{I}_{l, r, 0}\right)_{j}=\left(\bar{I}_{l, r, 0}\right)_{j-1}+0.5\left(\left(\bar{I}_{l, r}\right)_{j-1}+\left(\bar{I}_{l, r}\right)_{j}\right) h_{1}, \\
& \left(\bar{I}_{r, 0,0}\right)_{j}=\left(\bar{I}_{r, 0,0}\right)_{j-1}+0.5\left(\left(\bar{I}_{r, 0}\right)_{j-1}+\left(\bar{I}_{r, 0}\right)_{j}\right) h_{1}, \\
& \left(\bar{I}_{0, r, 0}\right)_{j}=\left(\bar{I}_{0, r, 0}\right)_{j-1}+0.5\left(\left(\bar{I}_{0, r}\right)_{j-1}+\left(\bar{I}_{0, r}\right)_{j}\right) h_{1}, \\
& \left(\bar{I}_{u, l, r}\right)_{j}=\left(\bar{I}_{u, l, r}\right)_{j-1}+0.5\left(\left(\bar{I}_{u, l}\right)_{j-1}+\left(\bar{I}_{u, l}\right)_{j}\right) \Delta_{j} g_{r}\left(h_{1}\right), \\
& \left(\bar{I}_{u, l, r, 0}\right)_{j}=\left(\bar{I}_{u, l, r, 0}\right)_{j-1}+0.5\left(\left(\bar{I}_{u, l, r}\right)_{j-1}+\left(\bar{I}_{u, l, r}\right)_{j}\right) h_{1},
\end{aligned}
$$

for all $u, l, r \in[1, q]$. It must be noted that the above set of equations would have to be solved in the same hierarchical order as shown. For instance, to solve for $\left(\bar{I}_{l, r, 0}\right)_{j}$ using the second of the above set of equations, one must first solve for $\left(\bar{I}_{l, r}\right)_{j}$ using the first equation. At this stage, given the fact that the above Heun scheme has a global accuracy order of $\mathrm{O}\left(h_{1}\right)$, it is required to determine the appropriate value of the step size $h_{1}$ in terms of the original time step size $h$, so that the desired respective local error orders of $\mathrm{O}\left(h^{3}\right)$ and $\mathrm{O}\left(h^{2}\right)$ for displacement and velocity components are ideally maintained. Since the lowest order of a numerically approximated (using Eq. (41)) multiple integral that appears in the higher order Newmark velocity 
expansion is $I_{l, r}$ and one has the relation (using Eq. (40)) $I_{l, r}=h \bar{I}_{l, r}$, it directly follows from Proposition 2 that one must have $h_{1}=h^{1.5}$ to maintain the $\mathrm{O}\left(h^{2}\right)$ local error in velocity. Similarly, from displacement point of view, the lowest order of a numerically approximated (using Eq. (41)) multiple integral in the higher order Newmark scheme is $I_{l, r, 0}$. Since $I_{l, r, 0}=h^{2} \bar{I}_{l, r, 0}$, one again derives via Proposition 2 that $h_{1}=h^{1.5}$ so that the local error remains $\mathrm{O}\left(h^{3}\right)$. However, in the special case of the multiplicative noise coefficients, $\sigma_{r}^{(j)}(j=1,2, \ldots, n)$, not being explicit functions of the velocity vector $X_{2}=\left\{x_{2}^{(j)}\right\}$ (and this is the case with most of the engineering systems), the choice of $h_{1}=h$ is enough to maintain the desired error orders. All linear and non-linear engineering systems with only additive random noises also fall within this category.

Still higher order stochastic Newmark maps may be theoretically derived, but it should be clear from the above discussion that the associated numerical difficulties increase too sharply to make such maps practically useful in general. Thus consider a higher order Newmark scheme of $\mathrm{O}\left(h^{3}\right)$ in velocity components. As in the previous cases, one has to numerically obtain scaled multiple integrals of the type $\bar{I}_{l, r}(1)$, where $l, r \neq 0, l \neq r$. If a Heun scheme with step size $h_{1}$ is again used for this purpose, one must necessarily use $h_{1}=h^{2.5}$ to arrive at $\mathrm{O}\left(h^{3}\right)$ local accuracy order in velocity. For instance, if $h=0.01$ then one has $h_{1}=0.00001$, i.e., it is needed to model $10^{5}$ random variables for each step of the original Newmark map and this is indeed an enormous numerical task.

Finally, it is worth noting that the following scheme may be adopted to generate Wiener increments, $\Delta_{i} W_{r}(s), r=1,2, \ldots, q$, for implementing the stochastic Newmark algorithms. To begin with, $r$ sets of independent and $N(0,1)$ random variables, $S_{r}=\left\{\varpi_{1}^{(r)}, \varpi_{2}^{(r)}, \ldots, \varpi_{i}^{(r)}, \ldots\right\}$, are generated. These Gaussian variables may be obtained from uniformly distributed pseudo-random variables in [0,1] via Box-Muller or Polar-Marsaglia transformations. Next, the desired Wiener increments are generated via the scaling: $\Delta_{i} W_{r}(s)=\varpi_{i}^{(r)} \sqrt{s}$.

\subsection{Numerical examples}

The basic aim of the present paper is the theoretical derivation of a class of useful stochastic Newmark algorithms, and as such a limited set of illustrations are presented in this section for a non-linear, hardening (single-well) Duffing oscillator under additive, multiplicative and filtered white noise excitations. In addition to being a workhorse example for single-degree-of-freedom (SDOF) non-linear engineering systems, the Duffing equation is known to behave very much similar to a large class of non-linear problems in structural dynamics.

Example 1. For the first example, consider the non-linear second order stochastic differential equation (SDE) for Duffing oscillator under a deterministic sinusoidal and an additive white noise inputs. Such a system is adequately described by the following five-parameter equation [15]:

$$
\ddot{x}+2 \pi \varepsilon_{1} \dot{x}+4 \pi^{2} \varepsilon_{2}\left(1+x^{2}\right) x=4 \pi^{2} \varepsilon_{3} \cos (2 \pi t)+4 \pi^{2} \varepsilon_{4} \dot{W}_{1}(t) .
$$

Since $\dot{W}_{1}(t)$ is physically meaningless (in the sense of sample paths), the SDE may be more properly written in the following incremental state-space form:

$$
\begin{aligned}
& \mathrm{d} x_{1}(t)=x_{2}(t) \mathrm{d} t \\
& \mathrm{~d} x_{2}(t)=\left(-2 \pi \varepsilon_{1} x_{2}-4 \pi^{2} \varepsilon_{2}\left(1+x_{1}^{2}\right) x_{1}+4 \pi^{2} \varepsilon_{3} \cos (2 \pi t)\right) \mathrm{d} t+4 \pi^{2} \varepsilon_{4} \mathrm{~d} W_{1}(t) .
\end{aligned}
$$

Using Eqs. (10) and (11) with $n=1$, one obtains the following lower order Newmark approximation $\widetilde{X}=\left\{\tilde{x}_{1, i}, \tilde{x}_{2, i}\right\}^{\mathrm{T}}$ for the desired solution $\widehat{X}=\left\{x_{1, i}, x_{2, i}\right\}^{\mathrm{T}}$ :

$$
\begin{aligned}
& \tilde{x}_{1, i}=x_{1, i-1}+x_{2, i-1} h+4 \pi^{2} \varepsilon_{4} I_{1,0}+\alpha a\left(\widehat{X}_{i-1}, t_{i-1}\right) \frac{h^{2}}{2}+(1-\alpha) a\left(\widetilde{X}_{i}, t_{i}\right) \frac{h^{2}}{2}, \\
& \tilde{x}_{2, i}=x_{2, i-1}+4 \pi^{2} \varepsilon_{4} I_{1}+\beta a\left(\widehat{X}_{i-1}, t_{i-1}\right) h+(1-\beta) a\left(\widetilde{X}_{i}, t_{i}\right) h
\end{aligned}
$$


where,

$$
a(\widehat{X}, t)=-2 \pi \varepsilon_{1} x_{2}-4 \pi^{2} \varepsilon_{2}\left(x_{1}+x_{1}^{3}\right)+4 \pi^{2} \varepsilon_{3} \cos (2 \pi t) .
$$

Note that the superscript $(j)$ has been omitted from the state variables since $n=1$. As is clear from the above equations, a specific advantage of the lower order scheme is its simplicity. Moreover, no laborious numerical generation of multiple integrals are involved. If one intends to use the higher order Newmark scheme for the above problem, then Eqs. (14) and (17) may respectively be used to determine the associated implicit maps for displacement and velocity components. This leads to:

$$
\begin{aligned}
\tilde{x}_{1, i}= & x_{1, i-1}+x_{2, i-1} h+4 \pi^{2} \varepsilon_{4} I_{1,0}+a\left(\widehat{X}_{i-1}, t_{i-1}\right) \frac{h^{2}}{2}-8 \pi^{3} \varepsilon_{1} \varepsilon_{4} I_{1,0,0}+\alpha \operatorname{La}\left(\widehat{X}_{i-1}, t_{i-1}\right) \frac{h^{3}}{6} \\
& +(1-\alpha) \operatorname{La}\left(\widetilde{X}_{i}, t_{i}\right) \frac{h^{3}}{6}, \\
\tilde{x}_{2, i}= & x_{2, i-1}+4 \pi^{2} \varepsilon_{4} I_{1}+a\left(\widehat{X}_{i-1}, t_{i-1}\right) h-8 \pi^{3} \varepsilon_{1} \varepsilon_{4} I_{1,0}+\beta L a\left(\widehat{X}_{i-1}, t_{i-1}\right) \frac{h^{2}}{2}+(1-\beta) L a\left(\widetilde{X}_{i}, t_{i}\right) \frac{h^{2}}{2},
\end{aligned}
$$

where,

$$
L a\left(x_{1}, x_{2}, t\right)=-2 \pi \varepsilon_{1} a\left(x_{1}, x_{2}, t\right)-4 \pi^{2} \varepsilon_{2}\left(1+3 x_{1}^{2}\right) x_{2}-8 \pi^{3} \varepsilon_{3} \sin (2 \pi t),
$$

and the expression for the drift coefficient $a\left(x_{1}, x_{2}, t\right)$ is still given by Eq. (43c). Compared with the lower order case, the only additional multiple integral to be modeled here is $I_{1,0,0}$. This may be readily done following the scaling as detailed in the previous section followed by using a Heun map over [0,1] with $h_{1}=h$ to obtain for the scaled integral $\bar{I}_{1,0,0}(1)$, which in turn is related to $I_{1,0,0}(h)$ via $I_{1,0,0}(h)=h^{2.5} \bar{I}_{1,0,0}(1)$.

Another advantage of the stochastic Newmark algorithms is that the basic steps of its computer implementation as described above basically remains the same irrespective of the dimensionality of the engineering system. In case the system is non-linear (as in the present case), then for $\alpha, \beta \neq 1$ the associated Newmark maps constitute a set of coupled non-linear algebraic equations, which may be solved via a Newton-Raphson approach.

In order to compare Newmark solutions in the stochastic regime with those obtained via an acceptable stochastic numerical scheme, the stochastic Heun scheme (SHS) is adopted for this example. It is known that SHS has local and global truncation errors of $\mathrm{O}\left(h^{3 / 2}\right)$ and $\mathrm{O}(h)$ respectively [4], provided that the system is driven by only one white noise process. In case of more than one independently evolving white noise processes, local and global accuracy orders for SHS reduce to $\mathrm{O}(h)$ and $\mathrm{O}\left(h^{0.5}\right)$ respectively (i.e., the same as Euler method). It is also to be noted here that there exist certain stochastic Runge-Kutta schemes [4] which lead to a higher local error order, provided that certain very stringent equalities involving the first and second derivatives of the drift and diffusion vectors are satisfied. Indeed, these equalities are not satisfied for the hardening Duffing system (or, for that matter, for most other engineering systems), thereby leaving the SHS method as the most accurate known integration tool. Thus referring to the hardening Duffing SDE (42) under a single additive noise (wherein higher accuracy orders for SHS are maintained), one has the following map over the time interval $T_{i}=\left(t_{i-1}, t_{i}\right]$ to integrate the SDE based on SHS:

$$
\begin{aligned}
& z_{1, i}=x_{1, i-1}+0.5\left(x_{2, i-1}+\tilde{z}_{2, n}\right) h_{i}, \\
& z_{2, i}=x_{2, i-1}+0.5\left(\psi\left(x_{1, i-1}, x_{2, i-1}, t_{i-1}\right)+\psi\left(\tilde{z}_{1, i}, \tilde{z}_{2, i}, t_{i}\right)\right) h_{i}+4 \pi^{2} \varepsilon_{4} I_{1},
\end{aligned}
$$

where,

$$
\begin{aligned}
& h_{i}=t_{i}-t_{i-1}, \quad \tilde{z}_{1, i}=x_{1, i-1}+x_{2, i-1} h_{i}, \\
& \tilde{z}_{2, i}=x_{2, i-1}+\psi\left(x_{1, i-1}, x_{2, i-1}, t_{i-1}\right) h_{i}+\varepsilon_{4} I_{1}, \\
& \psi\left(x_{1}, x_{2}, t\right)=-2 \pi \varepsilon_{1} x_{2}-4 \pi^{2} \varepsilon_{2}\left(1+x_{1}^{2}\right) x_{1}+4 \pi^{2} \varepsilon_{3} \cos (2 \pi t) .
\end{aligned}
$$


In Eq. (45), $\left\{z_{1, i}, z_{2, i}\right\}^{\mathrm{T}}$ is the SHS approximation vector to $\left(x_{1, i}, x_{2, i}\right)$ and $\left\{x_{1, i-1}, x_{2, i-1}\right\}^{\mathrm{T}}$ constitute the known initial condition vector. In order to pathwise compare the results of lower and higher order stochastic Newmark methods (respectively acronymed as LSNM and HSNM) with that of SHS, it is needed to use the same realizations of standard Wiener increments, $I_{1}=\Delta_{i} W_{1}(h)$. A consistent time step size $h=0.01$ has been adopted in all the following numerical results. To understand the effect of arbitrary solution parameters, $\alpha$ and $\beta$, on LSNM and HSNM, displacement and velocity histories for a specific choice of parameters are plotted in Figs. 1 and 2 for different choices of these parameters in $[0,1]$. It can be seen that the trajectories do not sensitively depend on the choice of $\alpha$ and $\beta$. While choosing $\alpha=\beta=1$ makes the Newmark map explicit (and hence computationally faster), an implicit scheme is known to have far better stability characteristics, especially for larger time step sizes, and hence $\alpha=\beta=0.5$ has been consistently chosen in this study. In Figs. 3-5, displacement and velocity histories of LSNM and HSNM-based solutions of the oscillator under weak, medium and strong intensities of additive white noise inputs are shown. Sinusoidal deterministic inputs are assumed to be acting on the oscillator in these examples. Comparisons of time histories obtained via Newmark algorithms with those via SHS are also provided in these figures and they appear to be quite close. In fact, the deterministic forcing amplitude parameter $\varepsilon_{3}$ has been so chosen that there is, in the phase plane, an unsymmetrical (about the velocity axis) and dumb-bell shaped one-periodic orbit of the oscillator under no noise. Even though HSNM is the most accurate out of all the three methods employed, it is observed that LSNM works well in all the three cases, even under a strong additive noise
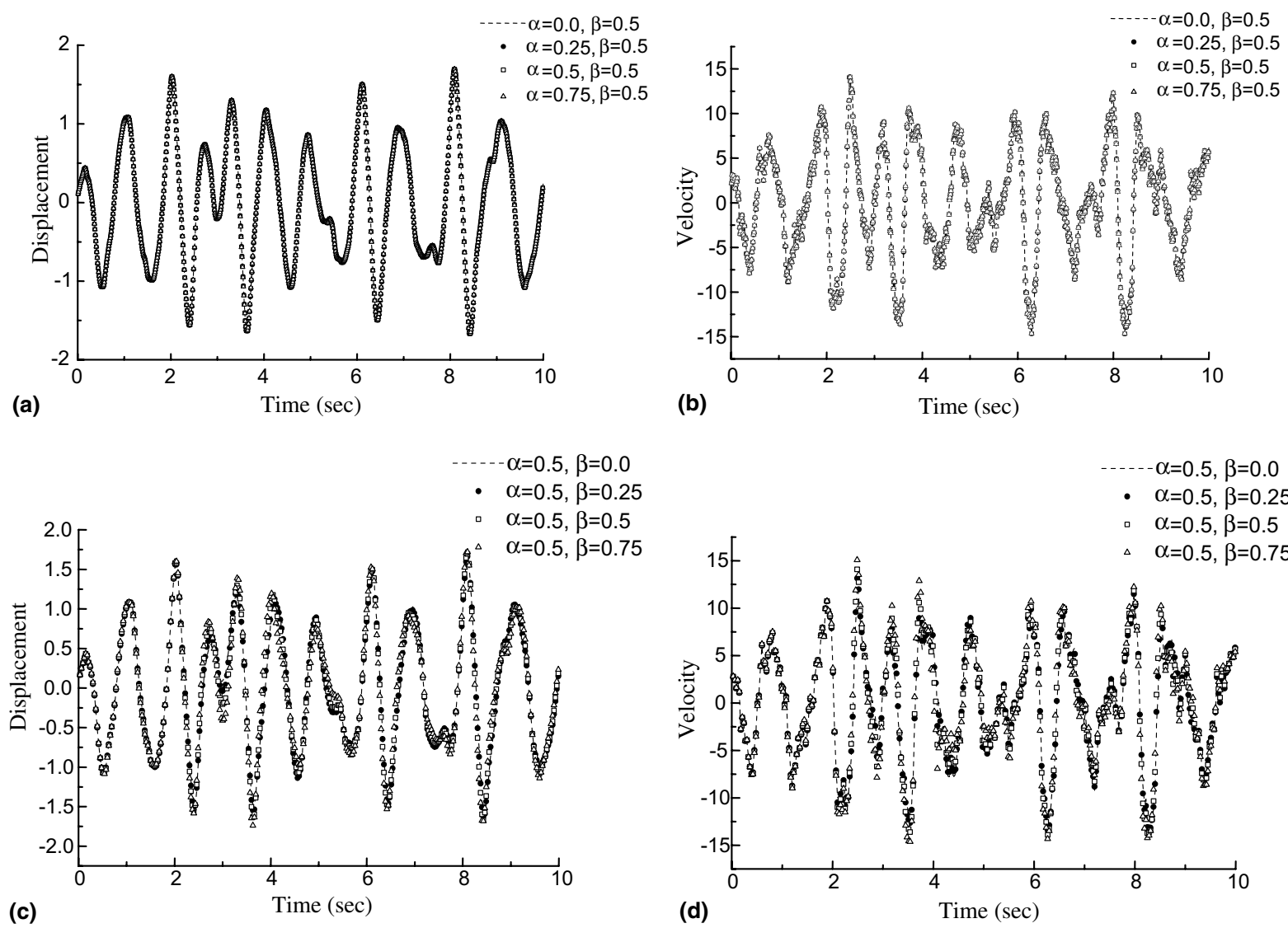

Fig. 1. Response histories under a single additive noise via LSNM for different $\alpha, \beta ; \varepsilon_{1}=0.25, \varepsilon_{2}=1.0, \varepsilon_{3}=1.0, \varepsilon_{4}=0.2$. 

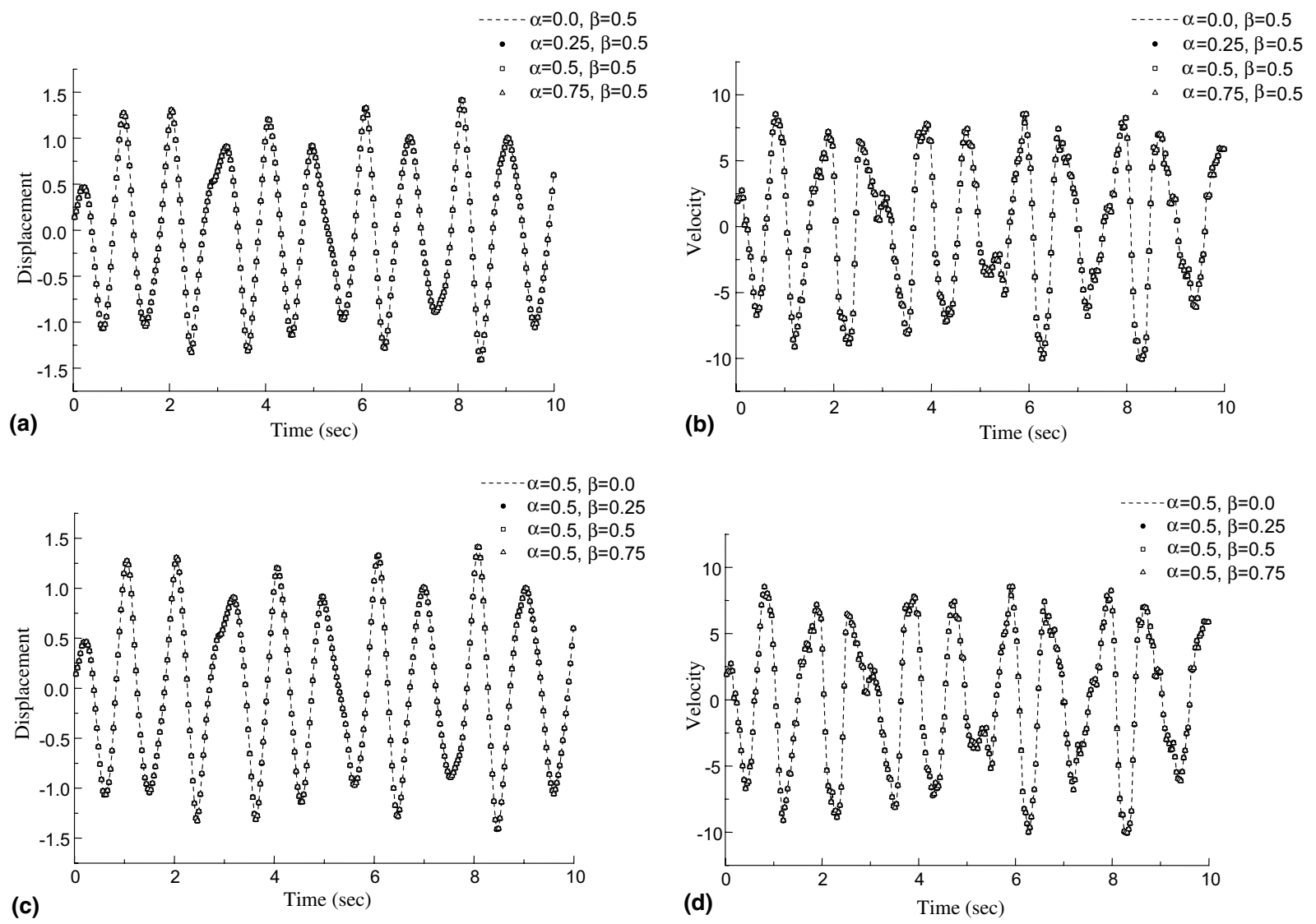

Fig. 2. Response histories under a single additive noise via HSNM for different $\alpha, \beta ; \varepsilon_{1}=0.25, \varepsilon_{2}=1.0, \varepsilon_{3}=1.0, \varepsilon_{4}=0.1$.
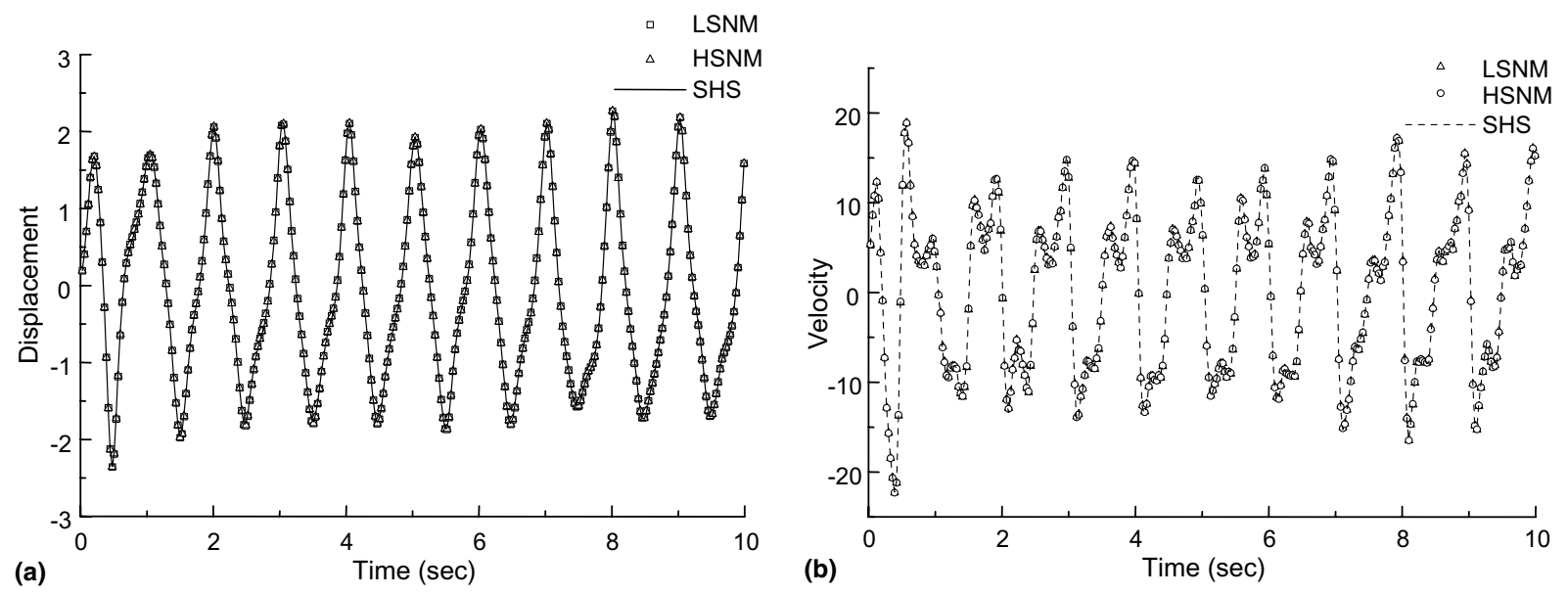

Fig. 3. Response histories under a single, weak-intensity additive noise via LSNM, HSNM and SHS: $\varepsilon_{1}=0.25, \varepsilon_{2}=1.0, \varepsilon_{3}=4.0$, $\varepsilon_{4}=0.1, \alpha=0.5, \beta=0.5$. 

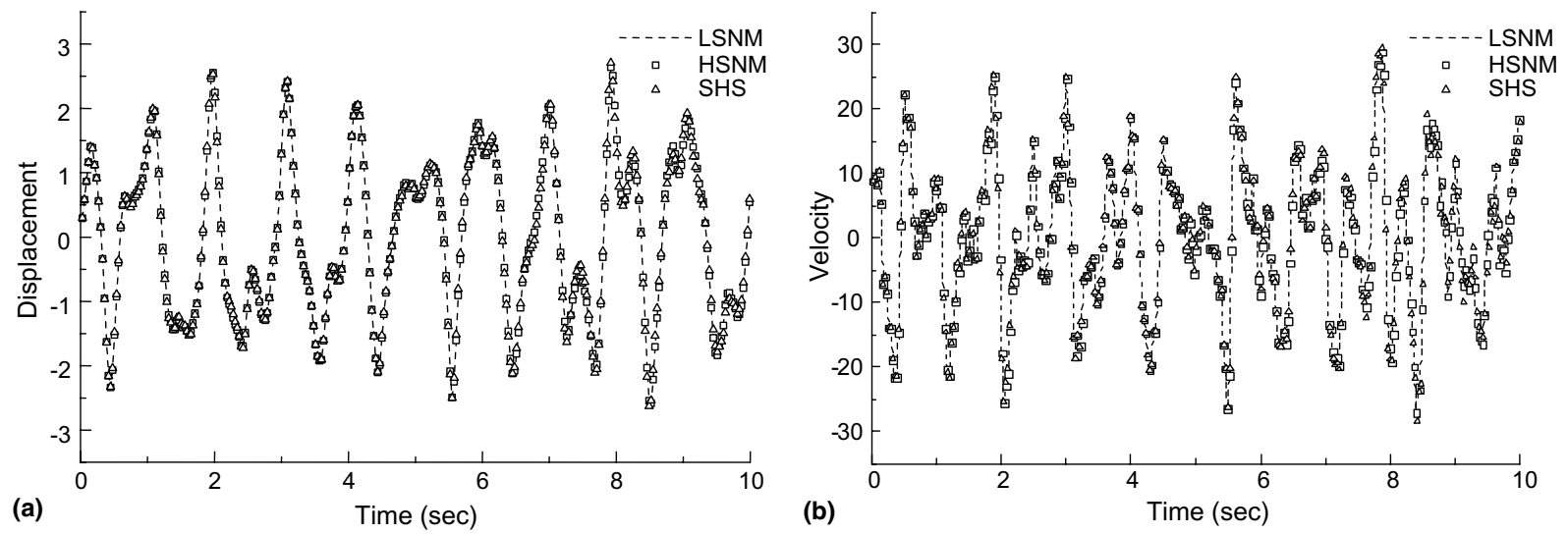

Fig. 4. Response histories under a single, medium-intensity additive noise via LSNM, HSNM and SHS: $\varepsilon_{1}=0.25, \varepsilon_{2}=1.0, \varepsilon_{3}=4.0$, $\varepsilon_{4}=0.5, \alpha=0.5, \beta=0.5$.
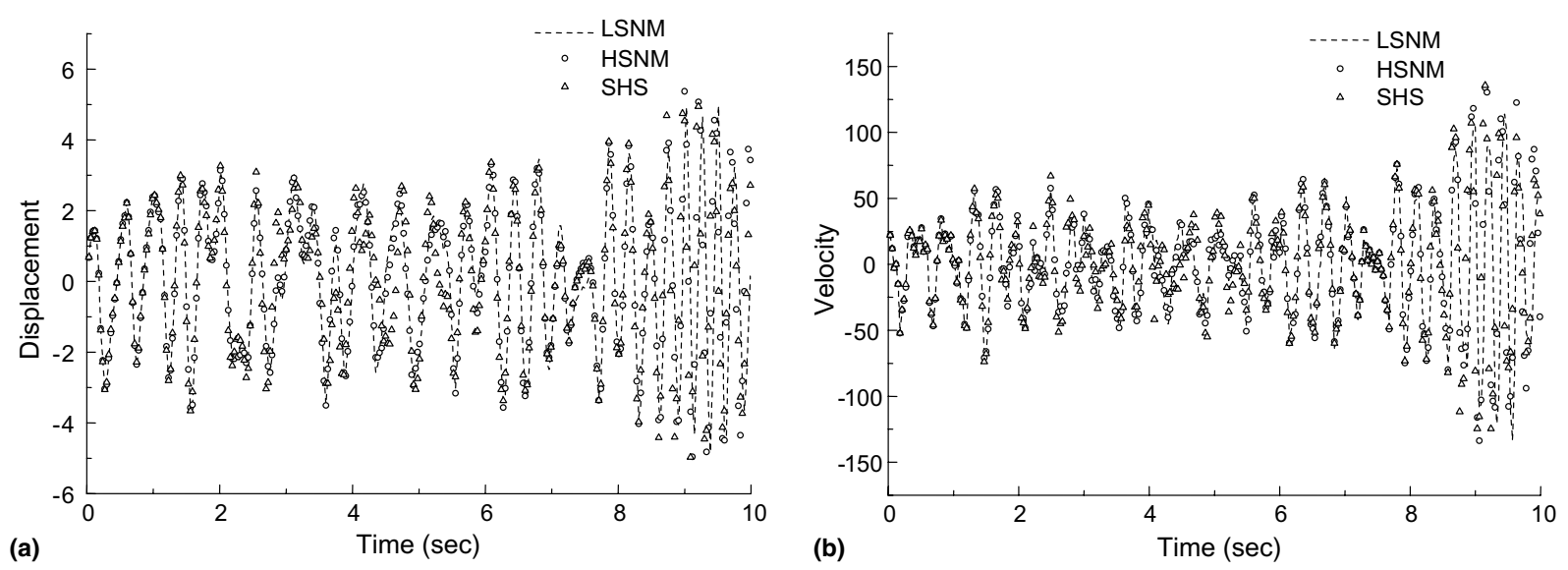

Fig. 5. Response histories under a single, high-intensity additive noise via LSNM, HSNM and SHS: $\varepsilon_{1}=0.25, \varepsilon_{2}=1.0, \varepsilon_{3}=4.0$, $\varepsilon_{4}=2.0, \alpha=0.5, \beta=0.5$.

intensity. In Fig. 6(a)-(c), the noisy phase plots of the orbit under weak, medium and strong additive noise intensities are plotted via HSNM. As seen from Fig. 6(b) and (c), the periodic structure of the orbit gets more and more diffused as the noise intensity grows. It is now of interest to see how the stochastic Newmark methods, especially the lower order one, behaves for locally unstable orbits, such as those encountered during chaos or quasi-periodicity. One such chaotic attractor under a weak additive stochastic excitation $\left(\varepsilon_{4}=0.5\right)$ is plotted in Fig. 7(a) and (b) using LSNM and HSNM respectively. In Fig. 8(a) and (b), phase plots of the strange attractor, respectively obtained via LSNM and HSNM, under a stronger additive noise intensity $\left(\varepsilon_{4}=20\right)$ are shown. Even though the global error in velocity computation is $\mathrm{O}\left(h^{1 / 2}\right)$ in LSNM as against $\mathrm{O}\left(h^{3 / 2}\right)$ in HSNM, the phase plots obtained for this kind of orbits via LSNM appear to be quite acceptable. In all the cases, however, one achieves a lower global displacement error of $\mathrm{O}\left(h^{3 / 2}\right)$ via LSNM than a global error order of $\mathrm{O}(h)$ in SHS.

It needs to be stressed that in case of multiple and independently evolving white noise inputs, there are no restrictions on the applicability of the Newmark procedure, even though SHS is generally not applicable with the same accuracy (as for a single noise input) in such cases. One such example is considered next. 

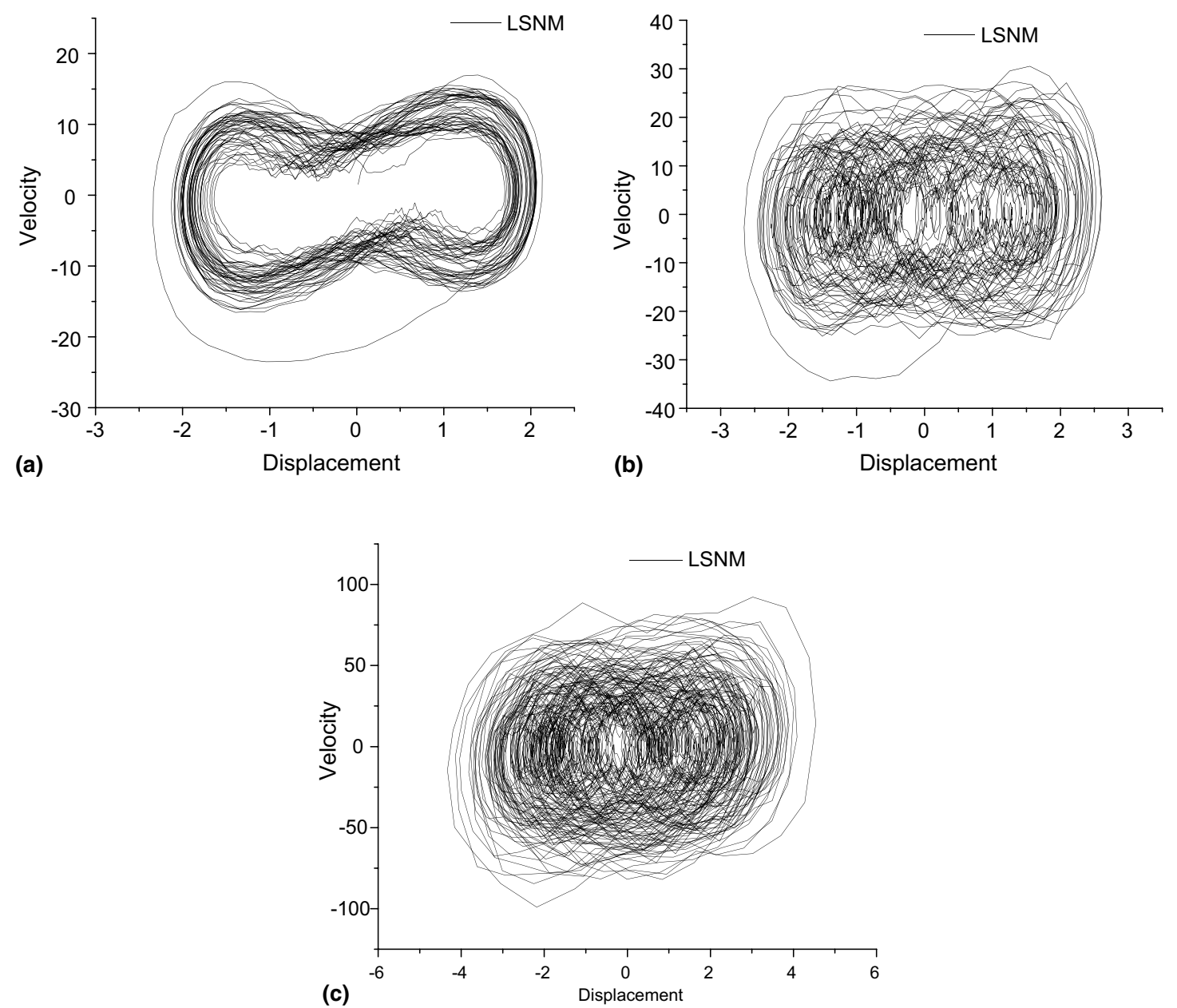

Fig. 6. Phase plots under a single additive noise via HSNM: $\varepsilon_{1}=0.25, \varepsilon_{2}=1.0, \varepsilon_{3}=4.0, \alpha=0.5, \beta=0.5$ : (a) weak noise intensity, $\varepsilon_{4}=0.1$; (b) medium noise intensity, $\varepsilon_{4}=0.5$; (c) high noise intensity, $\varepsilon_{4}=2.0$.

Example 2. Notwithstanding the simplicity of the LSNM, one has to exercise caution in assessing the accuracy of the method in certain cases, especially for multiplicative noise inputs, primarily due to its poor velocity approximation. The HSNM, on the other hand, may be relied upon to yield acceptably accurate results even in such cases. Consider, for instance, the hardening Duffing equation driven by combined additive and multiplicative noise excitations. The governing second order SDE takes the form:

$$
\begin{aligned}
& \mathrm{d} x_{1}(t)=x_{2}(t) \mathrm{d} t, \\
& \mathrm{~d} x_{2}(t)=a\left(x_{1}, x_{2}, t\right) \mathrm{d} t+4 \pi^{2}\left(\varepsilon_{4} \mathrm{~d} W_{1}(t)-\varepsilon_{5} x_{1} \mathrm{~d} W_{2}(t)\right),
\end{aligned}
$$

where $W_{1}(t)$ and $W_{2}(t)$ are two independently evolving Wiener processes. The lower order Newmark map for this equation over the time interval $T_{i}=\left[t_{i-1}, t_{i}=t_{i-1}+h\right)$ is given by:

$$
\begin{aligned}
& \tilde{x}_{1, i}=x_{1, i-1}+x_{2, i-1} h+4 \pi^{2}\left(\varepsilon_{4} I_{1,0}-\varepsilon_{5} x_{1, i-1} I_{2,0}\right)+\alpha\left\{a\left(\widehat{X}_{i-1}, t_{i-1}\right)+(1-\alpha) a\left(\widetilde{X}_{i}, t_{i}\right)\right\} \frac{h^{2}}{2}, \\
& \tilde{x}_{2, i}=x_{2, i-1}+4 \pi^{2}\left(\varepsilon_{4} I_{1}-\varepsilon_{5} x_{1, i-1} I_{2}\right)+\beta\left\{a\left(\widehat{X}_{i-1}, t_{i-1}\right) h+(1-\beta) a\left(\widetilde{X}_{i}, t_{i}\right)\right\} h .
\end{aligned}
$$




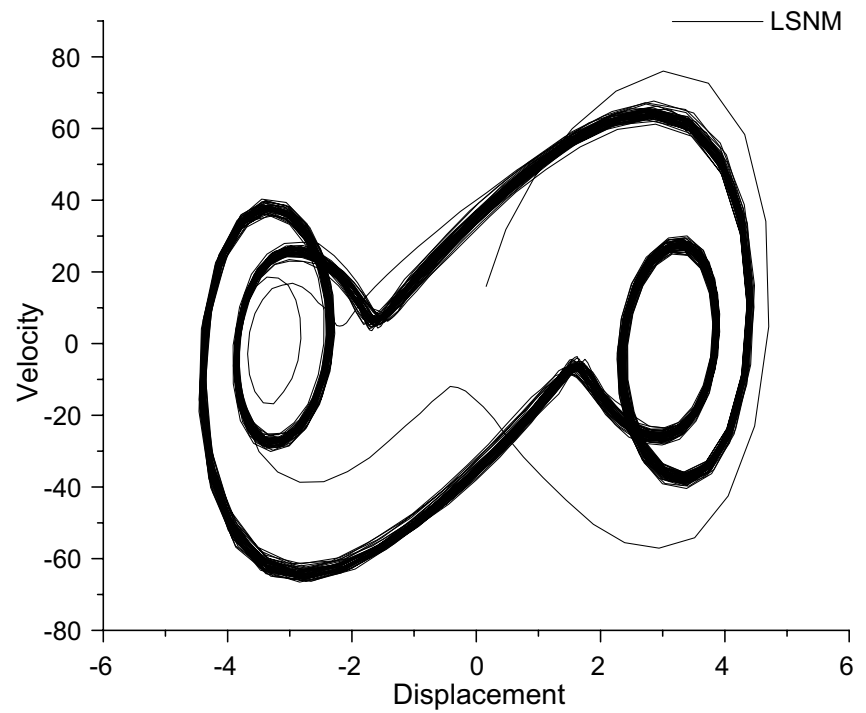

Fig. 7. Chaotic phase plots via LSNM under a single, weak-intensity additive noise: $\varepsilon_{1}=0.25, \varepsilon_{2}=1.0, \varepsilon_{3}=41.0, \varepsilon_{4}=0.1, \alpha=0.5$, $\beta=0.5$.

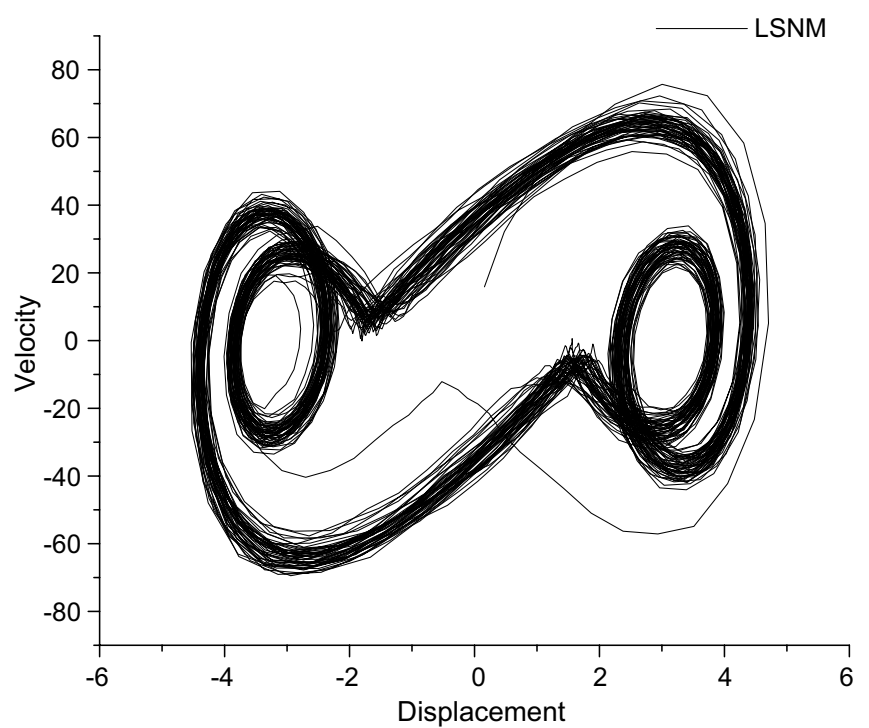

Fig. 8. Chaotic phase plots via LSNM under a single, medium-intensity additive noise: $\varepsilon_{1}=0.25, \varepsilon_{2}=1.0, \varepsilon_{3}=41.0, \varepsilon_{4}=0.25, \alpha=0.5$, $\beta=0.5$.

As in Example 1, one need not approximately model any multiple integral while employing LSNM here too. The higher order Newmark map for Eq. (47) over the same time interval takes the form: 


$$
\begin{aligned}
\tilde{x}_{1, i}= & x_{1, i-1}+x_{2, i-1} h+4 \pi^{2}\left(\varepsilon_{4} I_{1,0}-\varepsilon_{5} x_{1, i-1} I_{2,0}\right)+a\left(\widehat{X}_{i-1}, t_{i-1}\right) \frac{h^{2}}{2}+8 \pi^{3} \varepsilon_{1}\left(\varepsilon_{5} x_{1, i-1} I_{2,0,0}-\varepsilon_{4} I_{1,0,0}\right) \\
& -4 \pi^{2} \varepsilon_{5} x_{2, i-1} I_{0,2,0}+\left\{\alpha L a\left(\widehat{X}_{i-1}, t_{i-1}\right)+(1-\alpha) L a\left(\widetilde{X}_{i}, t_{i}\right)\right\} \frac{h^{3}}{6}, \\
\tilde{x}_{2, i}= & x_{2, i-1}+4 \pi^{2}\left(\varepsilon_{4} I_{1}-\varepsilon_{5} x_{1, i-1} I_{2}\right)-4 \pi^{2} \varepsilon_{5} x_{2, i-1} I_{0,2}+a\left(\widehat{X}_{i-1}, t_{i-1}\right) h \\
& -8 \pi^{3} \varepsilon_{1} \varepsilon_{4} I_{1,0}-8 \pi^{3} \varepsilon_{1} \varepsilon_{54} x_{1, i-1} I_{2,0}+\left\{\beta L a\left(\widehat{X}_{i-1}, t_{i-1}\right)+(1-\beta) L a\left(\widetilde{X}_{i}, t_{i}\right)\right\} h^{2} / 2 .
\end{aligned}
$$

As one can see, only three third level integrals need to be approximately modeled while implementing HSNM. These are $I_{1,0,0}, I_{2,0,0}$ and $I_{0,2,0}$. The procedure to do this has already been dealt with in detail and hence is not repeated here. As was mentioned previously, SHS is no better than the stochastic Euler scheme (SES) in the present case. The explicit stochastic map for Eq. (47) based on SES is given by:

$$
\begin{aligned}
& \hat{x}_{1, i}=x_{1, i-1}+x_{2, i-1} h, \\
& \hat{x}_{2, i}=x_{2, i-1}+4 \pi^{2}\left(\varepsilon_{4} I_{1}-\varepsilon_{5} x_{1, i-1} I_{2}\right)+a\left(x_{1, i-1}, x_{2, i-1}, t_{i-1}\right) h .
\end{aligned}
$$

In Figs. 9-11, displacement and velocity history plots via HSNM, LSNM and SES are shown for weak, medium and strong multiplicative noise intensities, while holding the additive noise intensity constant at low, medium and strong levels. Treating the results via HSNM as the reference (since this scheme is by far the most accurate), it is seen that while LSNM works reasonably well for weak and medium multiplicative noise intensities, it fares rather poorly for strong multiplicative intensities. The explicit SES scheme (having the same error order as the SHS for the present problem), on the other hand, behaves in a highly unstable manner and simply becomes unbounded within a short interval even for a low multiplicative noise intensity.

Even though more accurate, from the computational point of view HSNM is considerably slower than LSNM. One therefore sees that a certain amount of judgment, mostly based on an extensive numerical investigation, has to be exercised in deciding about the kind of stochastic Newmark algorithm to be applied to a given system.

Example 3. As a third example, consider a hardening Duffing oscillator under filtered white noise represented by the following stochastic differential equations (SDE-s).
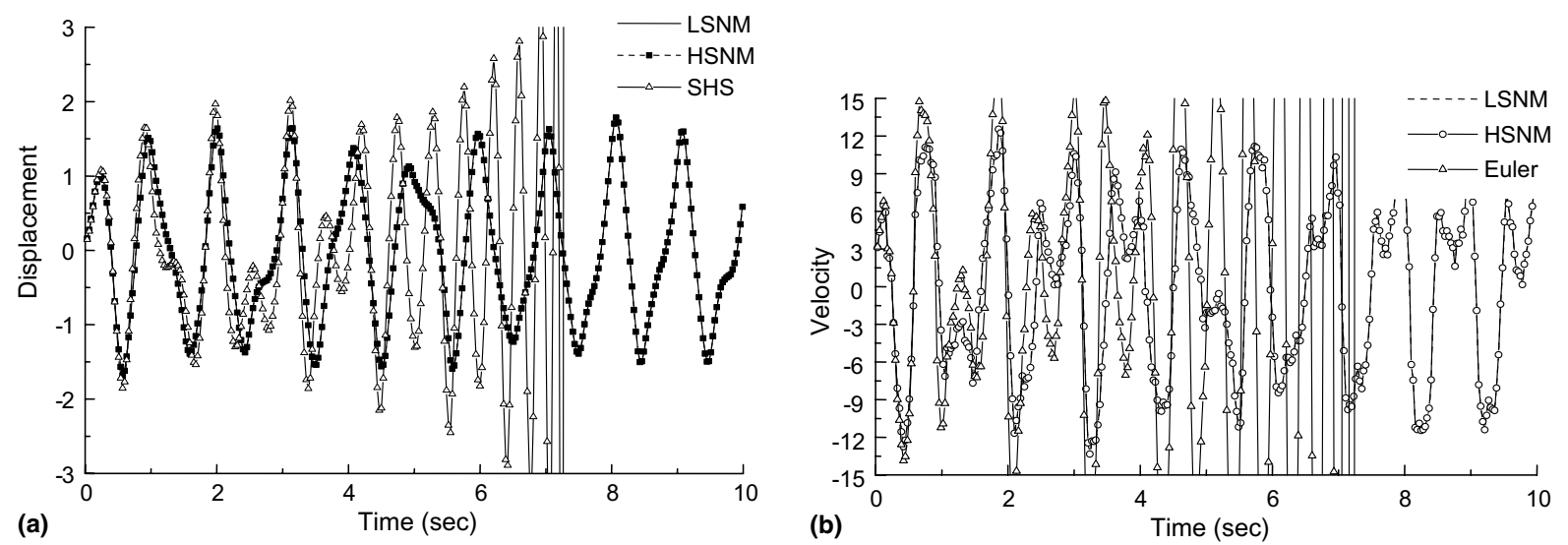

Fig. 9. Response histories under combined weak-intensity additive and weak-intensity multiplicative noise excitations via LSNM, HSNM and Euler method: $\varepsilon_{1}=0.25, \varepsilon_{2}=1.0, \varepsilon_{3}=41.0, \varepsilon_{4}=0.1, \varepsilon_{5}=0.1, \alpha=0.5, \beta=0.5$. 

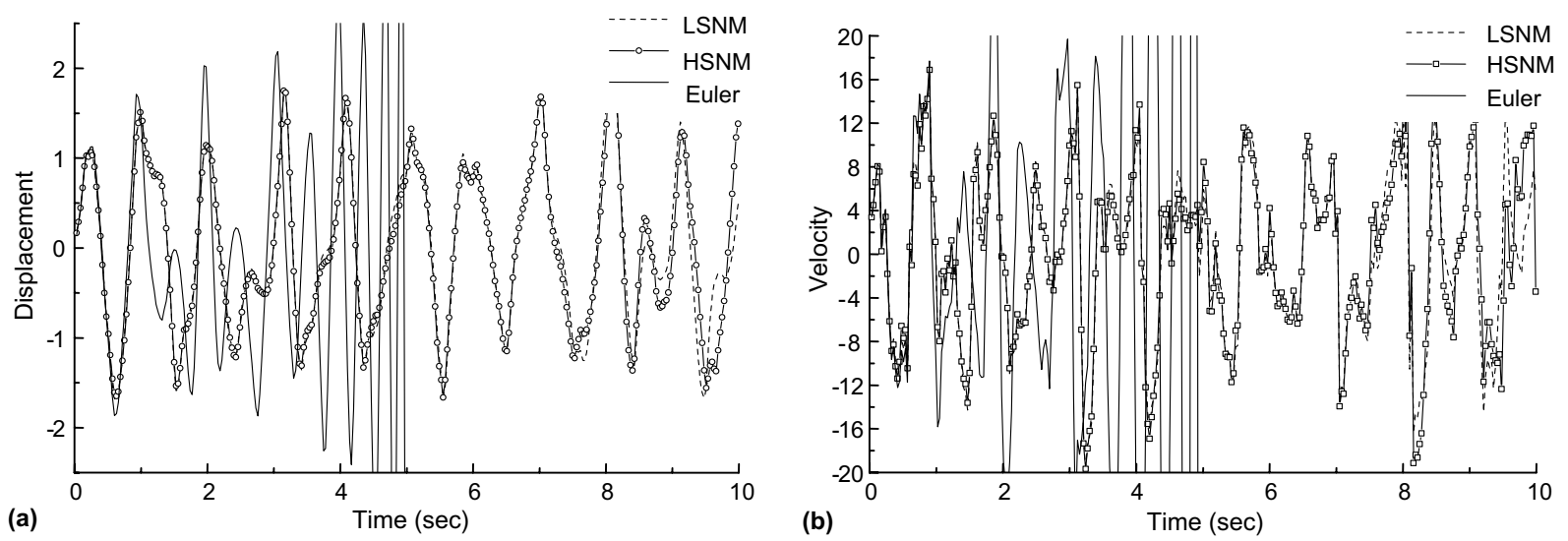

Fig. 10. Response histories under combined weak-intensity additive and medium-intensity multiplicative noise excitations via LSNM, HSNM and Euler method: $\varepsilon_{1}=0.25, \varepsilon_{2}=1.0, \varepsilon_{3}=41.0, \varepsilon_{4}=0.1, \varepsilon_{5}=0.5, \alpha=0.5, \beta=0.5$.
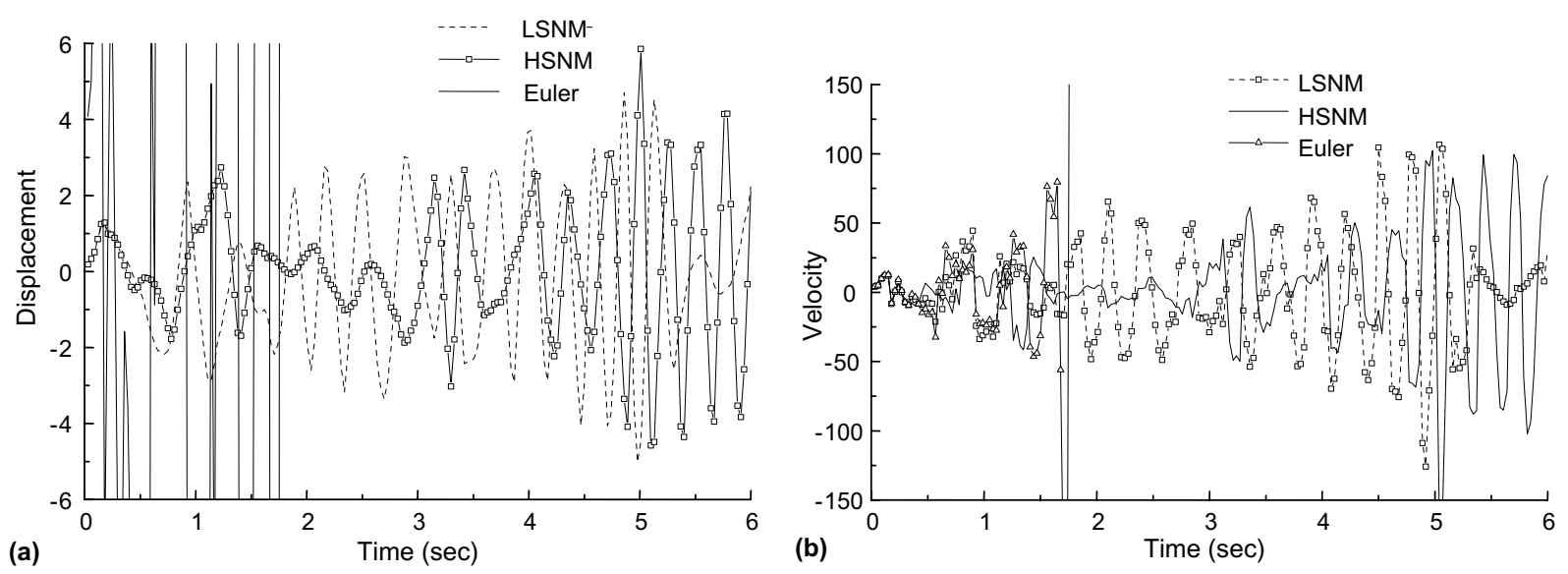

Fig. 11. Response histories under combined weak-intensity additive and high-intensity multiplicative noise excitations via LSNM, HSNM and Euler method: $\varepsilon_{1}=0.25, \varepsilon_{2}=1.0, \varepsilon_{3}=41.0, \varepsilon_{4}=0.1, \varepsilon_{5}=1.5, \alpha=0.5, \beta=0.5$.

$$
\begin{aligned}
& \ddot{x}+2 \pi \varepsilon_{1} \dot{x}+4 \pi^{2} \varepsilon_{2}\left(1+x^{2}\right) x=4 \pi^{2} \varepsilon_{3} \cos (2 \pi t)+g(t), \\
& \ddot{g}+2 \xi \omega \dot{g}+\omega^{2} g=4 \pi^{2} \varepsilon_{4} \dot{W}_{1},
\end{aligned}
$$

where, $\xi$ and $\omega$ in Eq. (51b) are damping coefficient and natural frequency respectively.

The SDE-s may be more properly written in the following incremental state-space form:

$$
\begin{aligned}
& \mathrm{d} x_{1}(t)=x_{2}(t) \mathrm{d} t, \\
& \mathrm{~d} x_{2}(t)=\left(-2 \pi \varepsilon_{1} x_{2}-4 \pi^{2} \varepsilon_{2}\left(1+x_{1}^{2}\right) x_{1}+4 \pi^{2} \varepsilon_{3} \cos (2 \pi t)+g(t)\right) \mathrm{d} t, \\
& \mathrm{~d} g_{1}(t)=g_{2}(t) \mathrm{d} t \\
& \mathrm{~d} g_{2}(t)=\left(-2 \xi \omega g_{2}-\omega^{2} g_{1}\right) \mathrm{d} t+4 \pi^{2} \varepsilon_{4} \mathrm{~d} W_{1}(t) .
\end{aligned}
$$



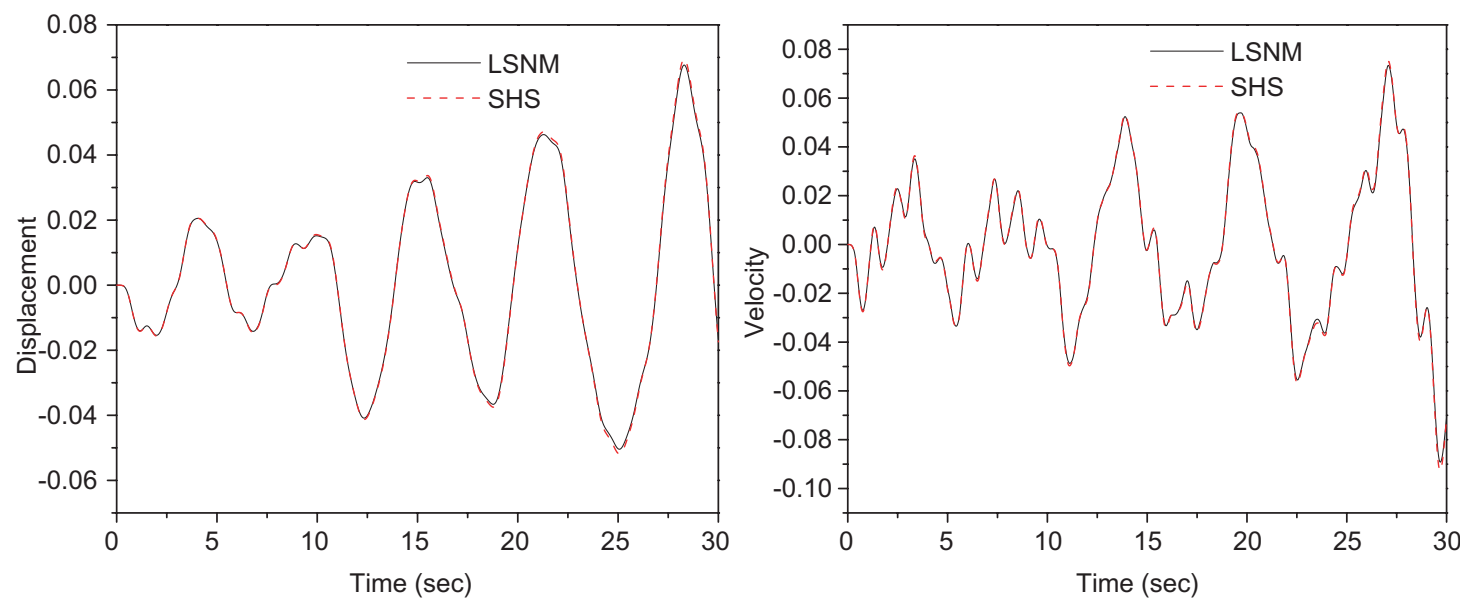

Fig. 12. Response histories under a low additive filtered white noise excitation via LSNM and SHS method: $\varepsilon_{1}=0.25, \varepsilon_{2}=1.0$, $\varepsilon_{3}=0.0, \varepsilon_{4}=0.5, \alpha=0.5, \beta=0.5$.

Using Eqs. (10) and (11) with $n=2$, one obtains the following lower order Newmark approximation $\widetilde{X}=\left\{\tilde{x}_{1, i}^{(1)}, \tilde{x}_{1, i}^{(2)}, \tilde{x}_{2, i}^{(1)}, \tilde{x}_{2, i}^{(2)}\right\}^{\mathrm{T}}$ for the desired solution $\widehat{X}=\left\{x_{1, i}^{(1)}, x_{1, i}^{(2)}, x_{2, i}^{(1)}, x_{2, i}^{(2)}\right\}^{\mathrm{T}}$ :

$$
\begin{aligned}
& \tilde{x}_{1, i}^{(1)}=x_{1, i-1}^{(1)}+x_{2, i-1}^{(1)} h+\alpha a^{(1)}\left(\widehat{X}_{i-1}, t_{i-1}\right) \frac{h^{2}}{2}+(1-\alpha) a^{(1)}\left(\widetilde{X}_{i}, t_{i}\right) \frac{h^{2}}{2}, \\
& \tilde{x}_{2, i}^{(1)}=x_{2, i-1}^{(1)}+\beta a^{(1)}\left(\widehat{X}_{i-1}, t_{i-1}\right) h+(1-\beta) a^{(1)}\left(\widetilde{X}_{i}, t_{i}\right) h, \\
& \tilde{x}_{1, i}^{(2)}=x_{1, i-1}^{(2)}+x_{2, i-1}^{(2)} h+4 \pi^{2} \varepsilon_{4} I_{1,0}+\alpha a^{(2)}\left(\widehat{X}_{i-1}, t_{i-1}\right) \frac{h^{2}}{2}+(1-\alpha) a^{(2)}\left(\widetilde{X}_{i}, t_{i}\right) \frac{h^{2}}{2}, \\
& \tilde{x}_{2, i}^{(2)}=x_{2, i-1}^{(2)}+4 \pi^{2} \varepsilon_{4} I_{1}+\beta a^{(2)}\left(\widehat{X}_{i-1}, t_{i-1}\right) h+(1-\beta) a^{(2)}\left(\widetilde{X}_{i}, t_{i}\right) h
\end{aligned}
$$

where,

$$
a^{(1)}(\widehat{X}, t)=-2 \pi \varepsilon_{1} x_{2}^{(1)}-4 \pi^{2} \varepsilon_{2}\left(x_{1}^{(1)}+x_{1}^{(1)^{3}}\right)+4 \pi^{2} \varepsilon_{3} \cos (2 \pi t)+x_{1}^{(2)},
$$

and,

$$
a^{(2)}(\widehat{X}, t)=-2 \xi \omega x_{2}^{(2)}-\omega^{2}\left(x_{1}^{(2)}\right) .
$$

Displacement and velocity plots (Eq. (51a)) have been obtained from Figs. 12-14 with increase in additive intensity via LSNM and SHS. Phase plots have been obtained in Fig. 15 for one medium $\left(\varepsilon_{4}=5.0\right)$ and the other strong $\left(\varepsilon_{4}=20.0\right)$ intensity additive noise. Both the results are in good agreement for all the response plots.

\section{Moment equations for linear systems}

In this section, the LSNM maps for displacement and velocity have been extended for linear MDOF dynamic structural systems to compute the mean square response in a direct way without a resort to expensive Monte Carlo Simulation (MCS). 

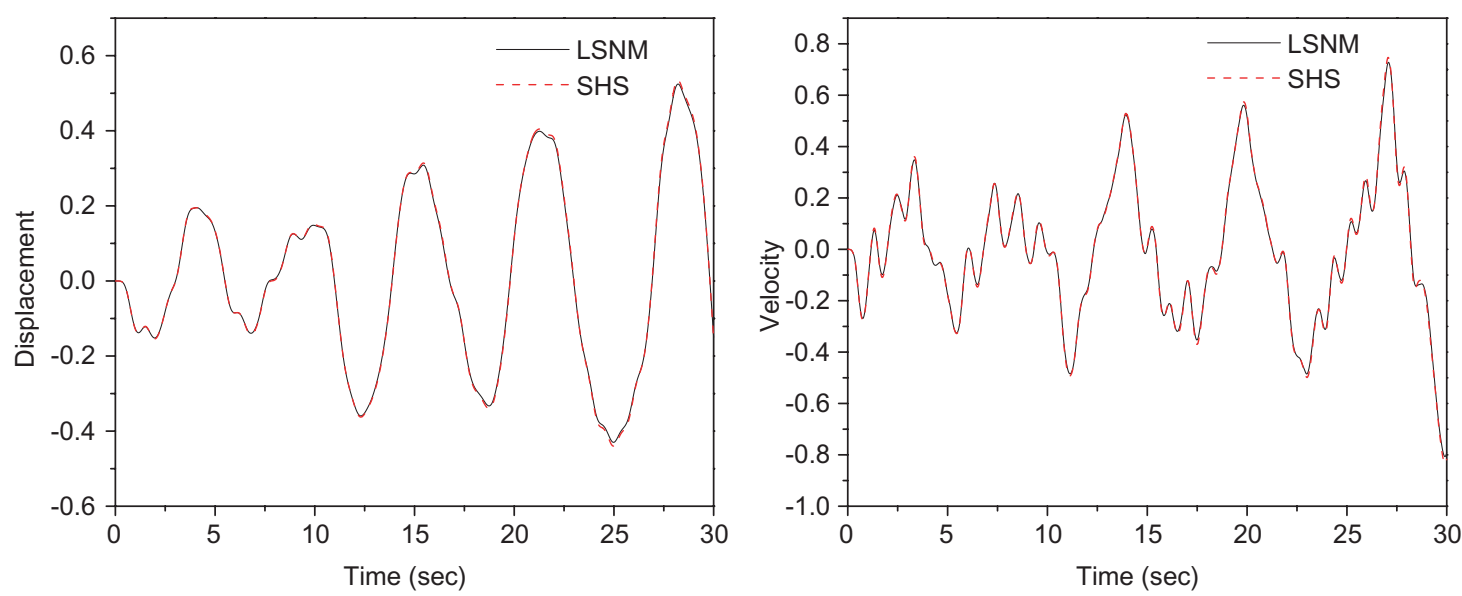

Fig. 13. Response histories under a medium additive filtered white noise excitation via LSNM and SHS method: $\varepsilon_{1}=0.25, \varepsilon_{2}=1.0$, $\varepsilon_{3}=0.0, \varepsilon_{4}=5.0, \alpha=0.5, \beta=0.5$.
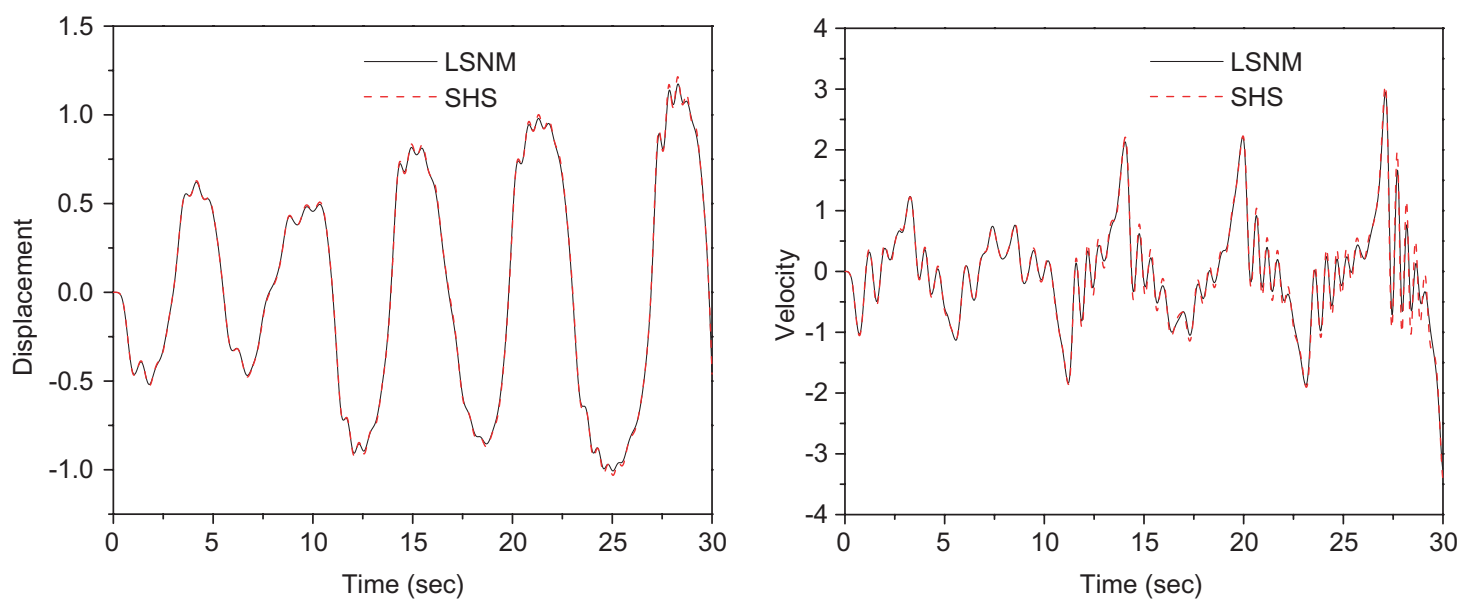

Fig. 14. Response histories under a strong additive filtered white noise excitation via LSNM \& SHS method: $\varepsilon_{1}=0.25, \varepsilon_{2}=1.0$, $\varepsilon_{3}=0.0, \varepsilon_{4}=20.0, \varepsilon_{5}=0.0, \alpha=0.5, \beta=0.5$.

\subsection{Formulation of the moment equations}

Thus, consider the governing equation in matrix form for a linear $n$-DOF system:

$$
M \ddot{X}+C \dot{X}+K X=\sum_{r=1}^{q} \sigma_{r}^{(j)}(t) \dot{W}_{r}(t)+F_{\mathrm{e}}(t), \quad j=1,2, \ldots, n,
$$

$\sum_{r=1}^{q} \sigma_{r}^{(j)}(t) \dot{W}_{r}(t)$ and $F_{\mathrm{e}}(t)$ denote input dynamic stochastic excitation vector and an external deterministic force vector respectively. Let the displacement vector be denoted as $X_{1}=\left\{x_{1}^{(j)} \mid j=1,2 \cdots n\right\}^{\mathrm{T}}$ and the velocity vector be denoted as $X_{2}=\left\{x_{2}^{(j)} \mid j=1,2 \cdots n\right\}^{\mathrm{T}}$. M, $C, K$ are system (globally assembled) mass, damping and stiffness matrices respectively. Via a suitable arrangement of the Newmark displacement 

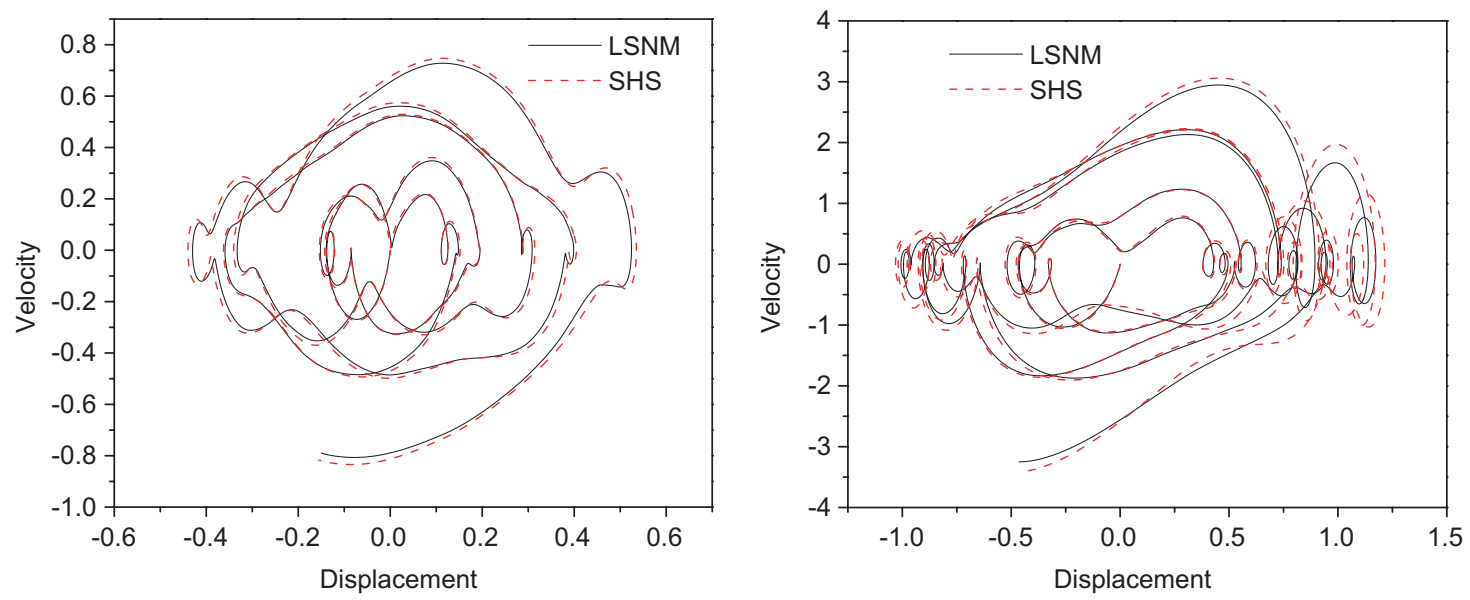

Fig. 15. Chaotic phase plots via LSNM \& SHS under additive filtered white noise: $\varepsilon_{1}=0.25, \varepsilon_{2}=1.0, \varepsilon_{3}=41.0, \alpha=0.5, \beta=0.5$ (a) $\varepsilon_{4}=5.0$, (b) $\varepsilon_{4}=20.0$.

and velocity maps for the lower order case (see Eqs. (10) and (11) without the remainder terms), one obtains after some algebraic manipulations:

$$
A \widetilde{X}_{i}=F_{\mathrm{e}} \widehat{X}_{i-1}+C_{1}+C_{2},
$$

where

$$
\begin{aligned}
& \widehat{X}=\left\{\begin{array}{l}
X_{1} \\
X_{2}
\end{array}\right\} \text { and } \tilde{X}=\left\{\begin{array}{l}
\widetilde{X}_{1} \\
\widetilde{X}_{2}
\end{array}\right\}, \\
& F_{\mathrm{e}}(t)=\left\{f_{\mathrm{e}}^{(j)}(t) \mid j=1,2 \cdots n\right\}^{\mathrm{T}} \\
& A=\left[\begin{array}{cc}
M+(1-\alpha) \frac{h^{2}}{2} K \quad(1-\alpha) \frac{h^{2}}{2} C \\
(1-\beta) h K & M+(1-\beta) h C
\end{array}\right], \\
& B=\left[\begin{array}{cc}
M-\alpha \frac{h^{2}}{2} K & h M-\alpha \frac{h^{2}}{2} C \\
-\beta h K & M-\beta h C
\end{array}\right], \\
& C_{1}=\left\{\begin{array}{l}
\sum_{r=1}^{q} \sigma_{r}^{(j)} I_{r 0} \\
\sum_{r=1}^{q} \sigma_{r}^{(j)} I_{r}
\end{array}\right\} \begin{array}{l}
\mid j=1,2 \cdots n \\
C_{2}=
\end{array} \\
& \left\{\begin{array}{l}
(1-\alpha) \frac{h^{2}}{2} F_{\mathrm{e}}\left(t_{i}\right) \\
(1-\beta) h F_{\mathrm{e}}\left(t_{i}\right)
\end{array}\right\}+\left\{\begin{array}{l}
\alpha \frac{h^{2}}{2} F_{\mathrm{e}}\left(t_{i-1}\right) \\
\beta h F_{\mathrm{e}}\left(t_{i-1}\right)
\end{array}\right\} .
\end{aligned}
$$

Post-multiplying both sides of Eq. (54) with their respective transposed vectors and taking the ensemble averages, the Newmark map for the co-variance matrix of the system may be derived as follows:

$$
R_{i}=N_{3} R_{i-1} N_{4}+N S_{i-1} N_{2}+N_{1} S_{i-1} N_{8}+N_{9} C_{1, i-1}^{\mathrm{T}} N_{2}+N_{10}
$$


where,

$$
\begin{aligned}
& R=\tilde{X} \tilde{X}^{\mathrm{T}}, \quad S=C_{1} C_{1}^{\mathrm{T}}, \\
& N_{1}=A^{-1}, \quad N_{2}=\left(A^{\mathrm{T}}\right)^{-1}, \quad N_{3}=N_{1} F_{\mathrm{e}}, \quad N_{4}=F_{\mathrm{e}}^{\mathrm{T}} N_{2}, \quad N_{5}=C_{2}, \quad N_{6}=C_{2}^{\mathrm{T}}, \\
& N_{7}=C_{2} C_{2}^{\mathrm{T}}, \quad N_{8}=N_{6} N_{2}, \quad N_{9}=N_{1} N_{5}, \quad N_{10}=N_{1} N_{7} N_{2} .
\end{aligned}
$$

In Eq. (56), $R$ stands for co-variance matrix of displacement and velocity vectors and $S$ stands for covariance matrix of stochastic load vector evaluated at the end of the previous time step.

\subsection{Applications to MDOF systems}

Consider a beam-column element with six degrees of freedom as shown in Fig. 16.

The nodal displacement for the member '1-2' shown in Fig. 16 can be represented as:

$$
\left\{u_{1} v_{1} \theta_{1} u_{2} v_{2} \theta_{2}\right\}^{\mathrm{T}}=\left\{x_{1}^{1} x_{1}^{2} x_{1}^{3} x_{2}^{1} x_{2}^{2} x_{2}^{3}\right\}^{\mathrm{T}} .
$$

In general, for a beam element $i-j$, the nodal displacement vector is given by:

$$
x_{1}^{(\mathrm{e})}=\left[x_{1}^{(3 i-2)} x_{1}^{(3 i-1)} x_{1}^{(3 i)} x_{1}^{(3 j-2)} x_{1}^{(3 j-1)} x_{1}^{(3 j)}\right]^{\mathrm{T}} .
$$

Similarly, the element nodal velocity vector is given by:

$$
x_{2}^{(\mathrm{e})}=\left[x_{2}^{(3 i-2)} x_{2}^{(3 i-1)} x_{2}^{(3 i)} x_{2}^{(3 j-2)} x_{2}^{(3 j-1)} x_{2}^{(3 j)}\right]^{\mathrm{T}} .
$$

The joint displacement and velocity vector is given by $\widehat{X}^{(\mathrm{e})}=\left\{\begin{array}{l}X_{1} \\ X_{2}\end{array}\right\}$.

The element equations are first formulated in the element coordinate system, and then transformed to the global coordinate system by standard procedure before element assemblage. Transformation of displacement and velocity parameters at nodes $j$ from element coordinates to global coordinates can be performed by standard transformation matrix given by:

$$
\mathbf{T}=\left[\begin{array}{ll}
t & 0 \\
0 & t
\end{array}\right]_{6 \times 6},
$$

where

$$
t=\left[\begin{array}{ccc}
\cos \theta & -\sin \theta & 0 \\
\sin \theta & \cos \theta & 0 \\
0 & 0 & 1
\end{array}\right],
$$

and $\theta$ is the angle of inclination of the local axis in an anticlockwise sense as positive angle from the global $X$-axis (Fig. 16).

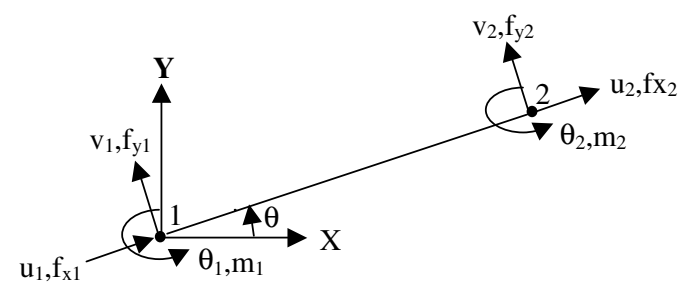

Fig. 16. Beam element in local co-ordinate (DOF per node $=3$ ), $X$ and $Y$ correspond to global direction. 


\subsection{Element matrices}

The element matrices required are stiffness matrix $k^{(\mathrm{e})}$, mass matrix $m^{(\mathrm{e})}$, and damping matrix $c^{(\mathrm{e})}$. The element stiffness matrix is formulated by superimposing the bending stiffness matrix $k_{\mathrm{b}}^{(\mathrm{e})}$ and geometric stiffness matrix $k_{\mathrm{g}}^{(\mathrm{e})}$ of the basic beam element and the stiffness matrix $k_{\mathrm{m}}^{(\mathrm{e})}$ of the linear bar element. The element mass matrix is obtained by superimposing $m_{\mathrm{t}}^{(\mathrm{e})}$ and $m_{\mathrm{r}}^{(\mathrm{e})}$ the consistent mass matrices of the basic beam element for lateral translational inertia and rotational inertia, respectively, and $m_{\mathrm{m}}^{(\mathrm{e})}$ the consistent mass matrix of the linear bar element. These matrices are given as follows.

(a) Bending stiffness matrix $k_{\mathrm{b}}^{(\mathrm{e})}$ :

$$
k_{\mathrm{b}}^{(\mathrm{e})}=\frac{E I}{L^{3}}\left[\begin{array}{cccc}
12 & 6 L & -12 & 6 L \\
6 L & 4 L^{2} & -6 L & 2 L^{2} \\
-12 & -6 L & 12 & -6 L \\
6 L & 2 L^{2} & -6 L & 4 L^{2}
\end{array}\right] .
$$

(b) Geometric stiffness matrix $k_{\mathrm{g}}^{(\mathrm{e})}$ :

$$
k_{\mathrm{g}}^{(\mathrm{e})}=\frac{f_{x 2}}{30 L}\left[\begin{array}{cccc}
36 & 3 L & -36 & 3 L \\
3 L & 4 L^{2} & -3 L & -L^{2} \\
-36 & -3 L & 36 & -3 L \\
3 L & -L^{2} & -3 L & 4 L^{2}
\end{array}\right] .
$$

(c) Axial stiffness matrix $k_{\mathrm{m}}^{(\mathrm{e})}$ :

$$
k_{\mathrm{m}}^{(\mathrm{e})}=\frac{A E}{L}\left[\begin{array}{cc}
1 & -1 \\
-1 & 1
\end{array}\right],
$$

where $L$ is the initial length of the beam axis, $E I$ is flexural rigidity. $f_{x 2}$ is the axial nodal force at node 2, and $A E$ is the axial rigidity. The degrees of freedom as relevant to the construction of $k_{\mathrm{b}}$ and $k_{\mathrm{g}}$ are $u_{\mathrm{b}}$ and those for constructing $k_{m}$ are $u_{\mathrm{m}}$. The vectors $u_{\mathrm{b}}$ and $u_{\mathrm{m}}$ are given by: $\left.u_{\mathrm{b}}=\begin{array}{llll}v_{1} & \theta_{1} & v_{2} & \theta_{2}\end{array}\right\}^{\mathrm{T}}$ and $u_{\mathrm{m}}=\left\{\begin{array}{ll}u_{1} & u_{2}\end{array}\right\}^{\mathrm{T}}$ with reference to Fig. 16 .

(d) Consistent mass matrix for lateral translation $m_{\mathrm{t}}^{(\mathrm{e})}$ :

$$
m_{\mathrm{t}}^{(\mathrm{e})}=\frac{\rho_{A} L}{420}\left[\begin{array}{cccc}
156 & 22 L & 54 & -13 L \\
22 L & 4 L^{2} & 13 L & -3 L^{2} \\
54 & 13 L & 156 & -22 L \\
-13 L & -3 L^{2} & -22 L & 4 L^{2}
\end{array}\right] .
$$

(e) Consistent mass matrix for rotation $m_{\mathrm{r}}^{(\mathrm{e})}$ :

$$
m_{\mathrm{r}}^{(\mathrm{e})}=\frac{\rho_{I}}{30 L}\left[\begin{array}{cccc}
36 & 3 L & -36 & 3 L \\
3 L & 4 L^{2} & -3 L & -L^{2} \\
-36 & -3 L & 36 & -3 L \\
3 L & -L^{2} & -3 L & 4 L^{2}
\end{array}\right] .
$$

(f) Consistent mass matrix for axial translation $m_{\mathrm{m}}^{(\mathrm{e})}$ :

$m_{\mathrm{m}}^{(\mathrm{e})}=\frac{\rho_{A} L}{6}\left[\begin{array}{ll}2 & 1 \\ 1 & 2\end{array}\right]$,

where $L$ is the undeformed length of beam axis; $\rho_{A}$ and $\rho_{I}$ are inertia constants and are defined as $\rho_{A}=\int_{A} \rho \mathrm{d} A$ and $\rho_{I}=\int_{A} \rho y^{2} \mathrm{~d} A$ where $\rho$ is the mass density, $y$ is the distance from the axis of centroid to the an infinitesimal area $\mathrm{d} A$ on the cross section. 
(g) Rayleigh Damping matrix $c^{(\mathrm{e})}$ :

$C_{i j}=a_{0}\left[M_{i j}\right]+a_{1}\left[K_{i j}\right], a_{0}$ and $a_{1}$ are proportionality constants.

\subsection{Numerical results and discussion}

Example 4. An SDOF system:

Consider an SDOF linear system given by its equation of motion as:

$\ddot{X}+2 \omega \xi \dot{X}+\omega^{2} X=\dot{W}(t)$.
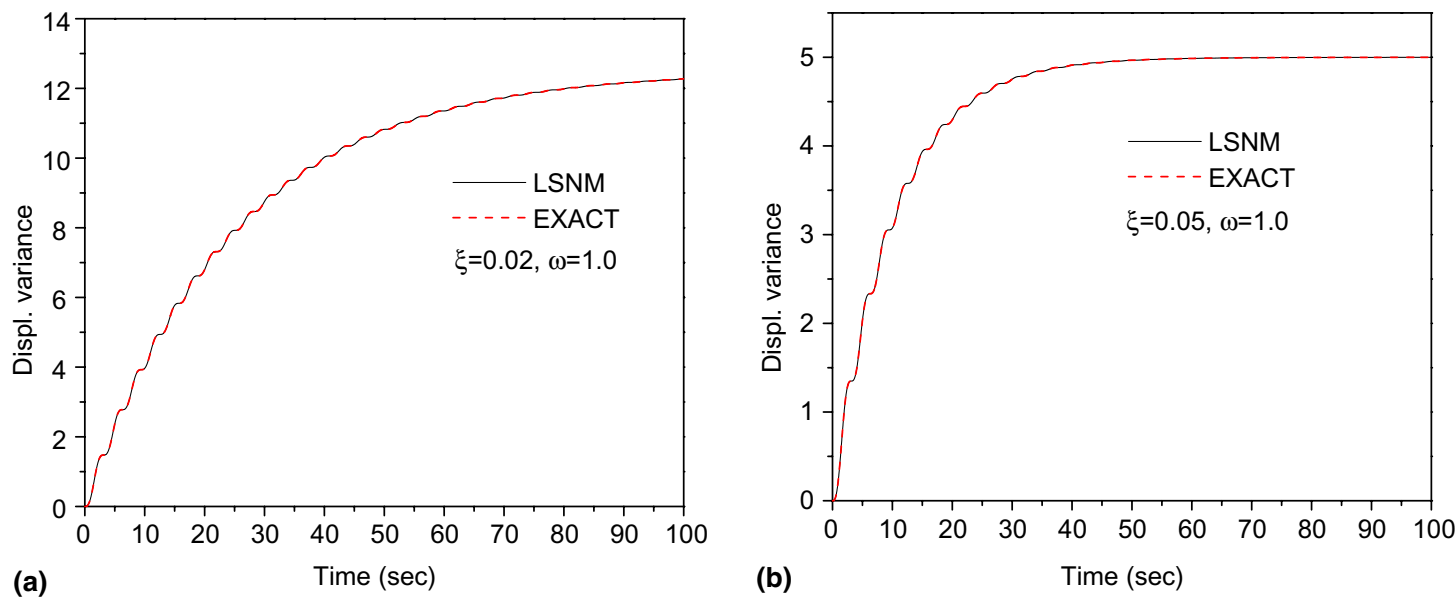

Fig. 17. Variance of displacement response of linear oscillator: $\omega=1.0$; (a) $\xi=0.02$, (b) $\xi=0.05$.
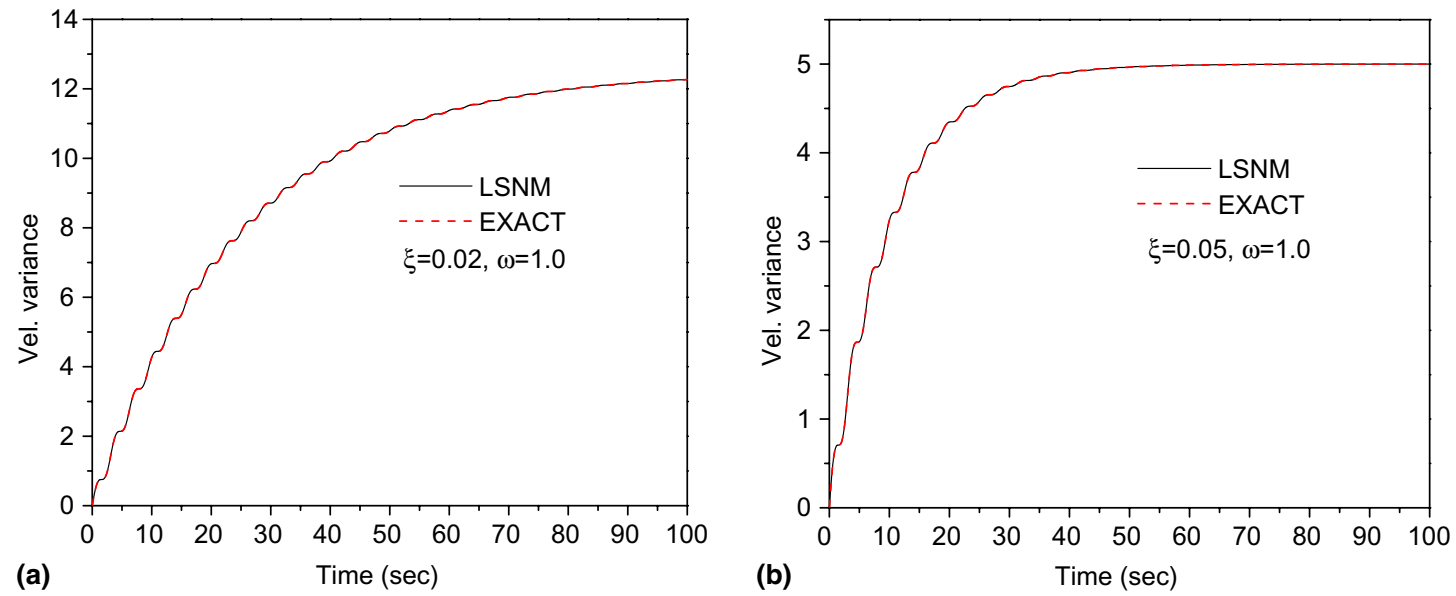

Fig. 18. Variance of velocity response of linear oscillator: $\omega=1.0$; (a) $\xi=0.02$, (b) $\xi=0.05$. 


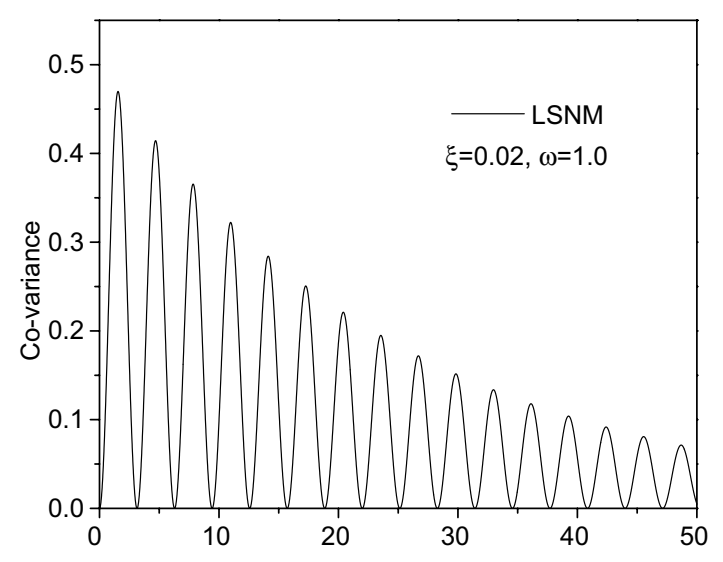

(a)

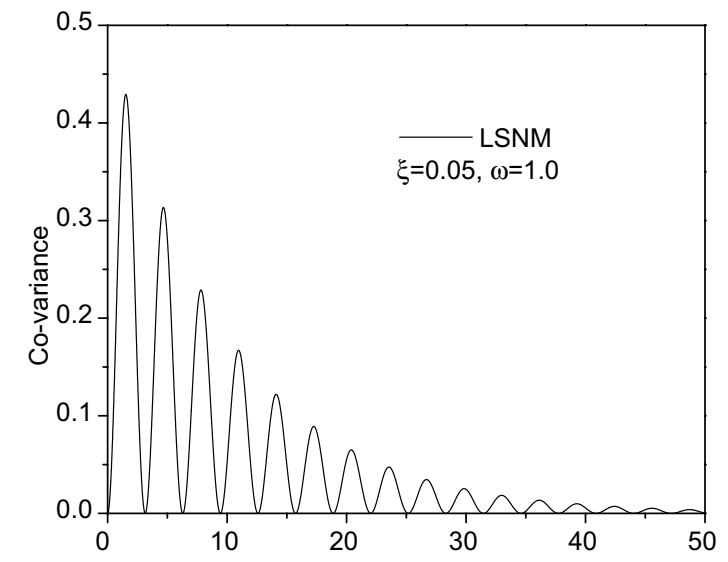

(b)

Time (sec)

Fig. 19. Covariance response of displacement and velocity of linear oscillator: $\omega=1.0 \mathrm{rad} / \mathrm{s}$; (a) $\xi=0.02$, (b) $\xi=0.05$.
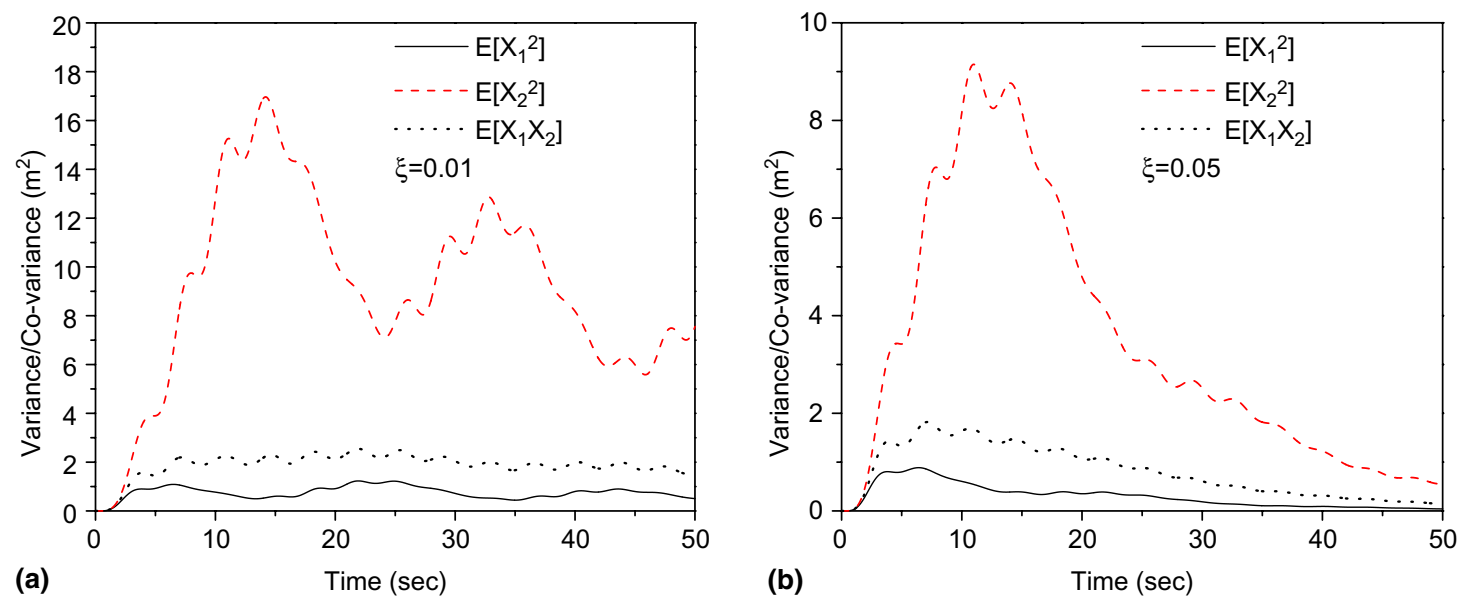

Fig. 20. Variance and covariance response of a 2-DOF system: (a) $\xi=0.01$, (b) $\xi=0.05$.

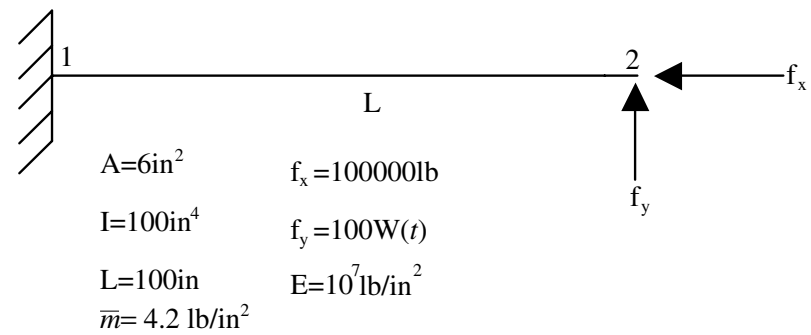

Fig. 21. A cantilever beam. 

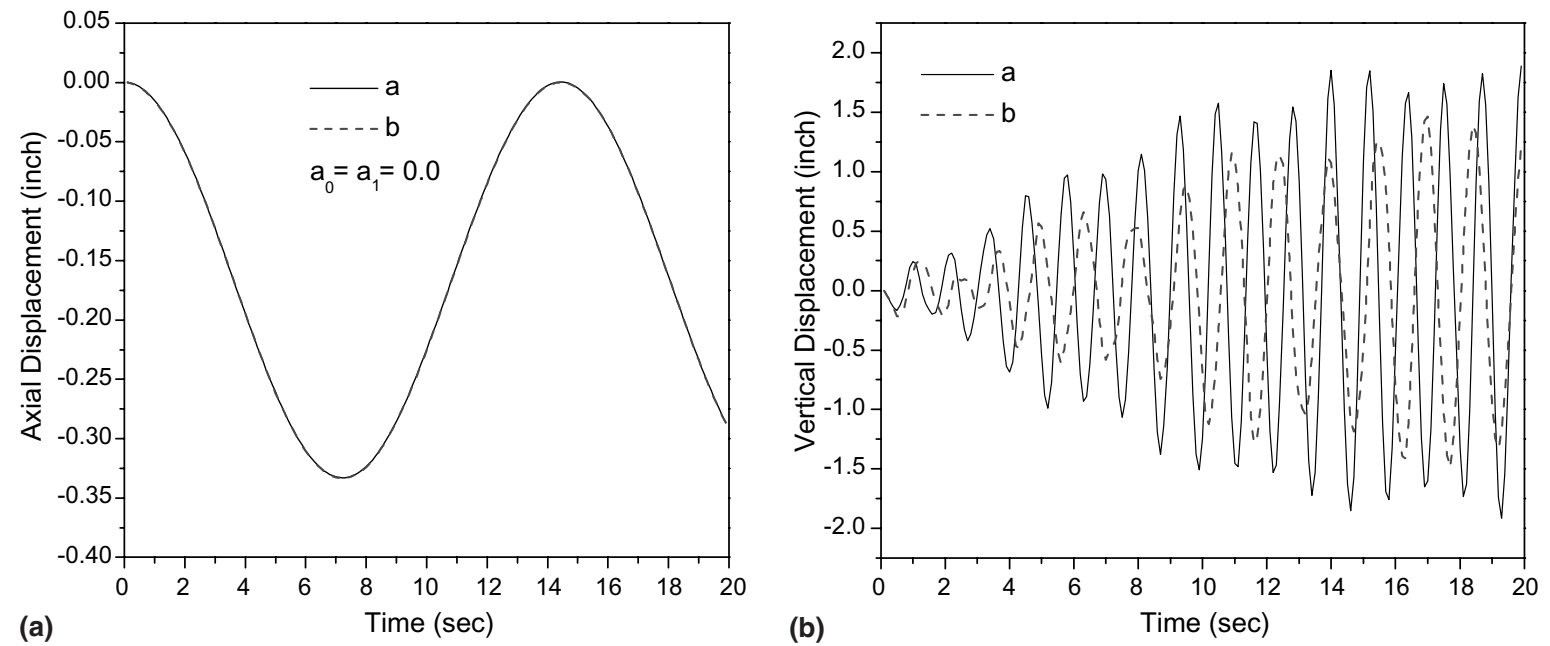

Fig. 22. Displacement response at the free end in axial and vertical direction without damping: (a) Without axial shortening and (b) with axial shortening.
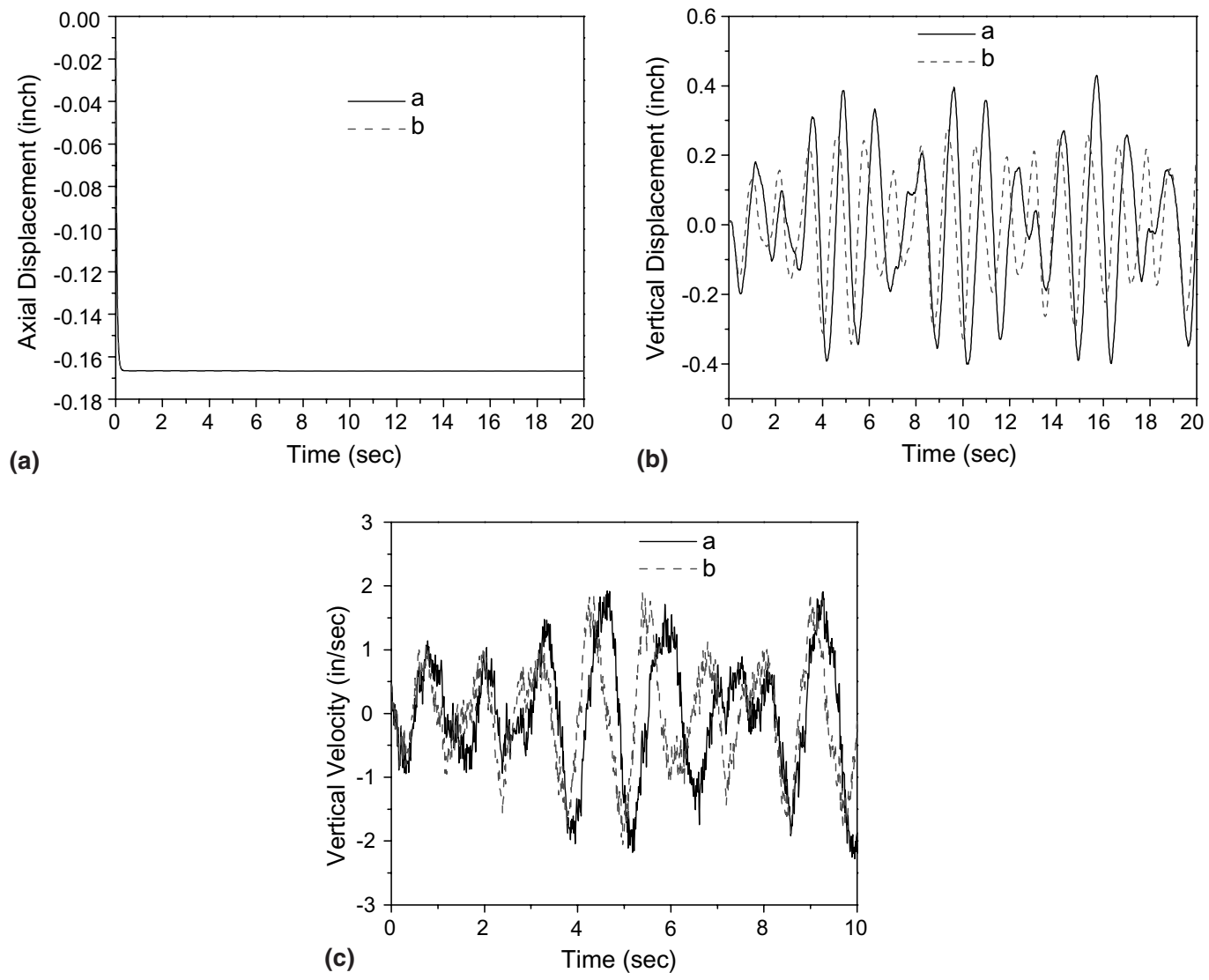

Fig. 23. Displacement and velocity response at the free end in axial and vertical direction with damping $\left(a_{0}=0.05, a_{1}=0.05\right)$; (a) Without axial shortening and (b) with axial shortening. 
Exact solutions for the transient displacement and velocity variance functions for the above equation had been reported by Caughey and Stumpf [3]. The variance and covariance evolutions of displacement and velocity have presently been obtained through the present method (SNM) and plotted in Figs. 1719. The results have been compared with exact solutions for two typical values of $\xi=0.02, \xi=0.05$ and for a fixed $\omega=1.0$. The results are in very good agreement with each other.

Example 5. A 2-DOF system:

Consider a two-degree-of freedom linear system modeled by the vector equations of motion:

$$
\left[\begin{array}{cc}
m_{1} & 0 \\
0 & m_{2}
\end{array}\right]\left\{\begin{array}{l}
\ddot{X}_{1} \\
\ddot{X}_{2}
\end{array}\right\}+\left[\begin{array}{cc}
c_{1}+c_{2} & -c_{2} \\
-c_{2} & c_{2}
\end{array}\right]\left\{\begin{array}{l}
\dot{X}_{1} \\
\dot{X}_{2}
\end{array}\right\}+\left[\begin{array}{cc}
k_{1}+k_{2} & -k_{2} \\
-k_{2} & k_{2}
\end{array}\right]\left\{\begin{array}{l}
X_{1} \\
X_{2}
\end{array}\right\}=\left\{\begin{array}{l}
F_{21} \\
F_{22}
\end{array}\right\} .
$$

The system parameters are: $m_{1}=1.0 \mathrm{~kg} ; m_{2}=0.1 \mathrm{~kg} ; k_{1}=1.0 \mathrm{~N} / \mathrm{m} ; k_{2}=0.1 \mathrm{~N} / \mathrm{m} ; \xi=0.05$.

The excitations are:

$$
\begin{aligned}
& F_{21}=[\exp (-0.1 t)-\exp (-1.5 t)] W_{21}, \\
& F_{22}=0.1 F_{21}
\end{aligned}
$$

where $W_{21}$ is a Gaussian white-noise process.
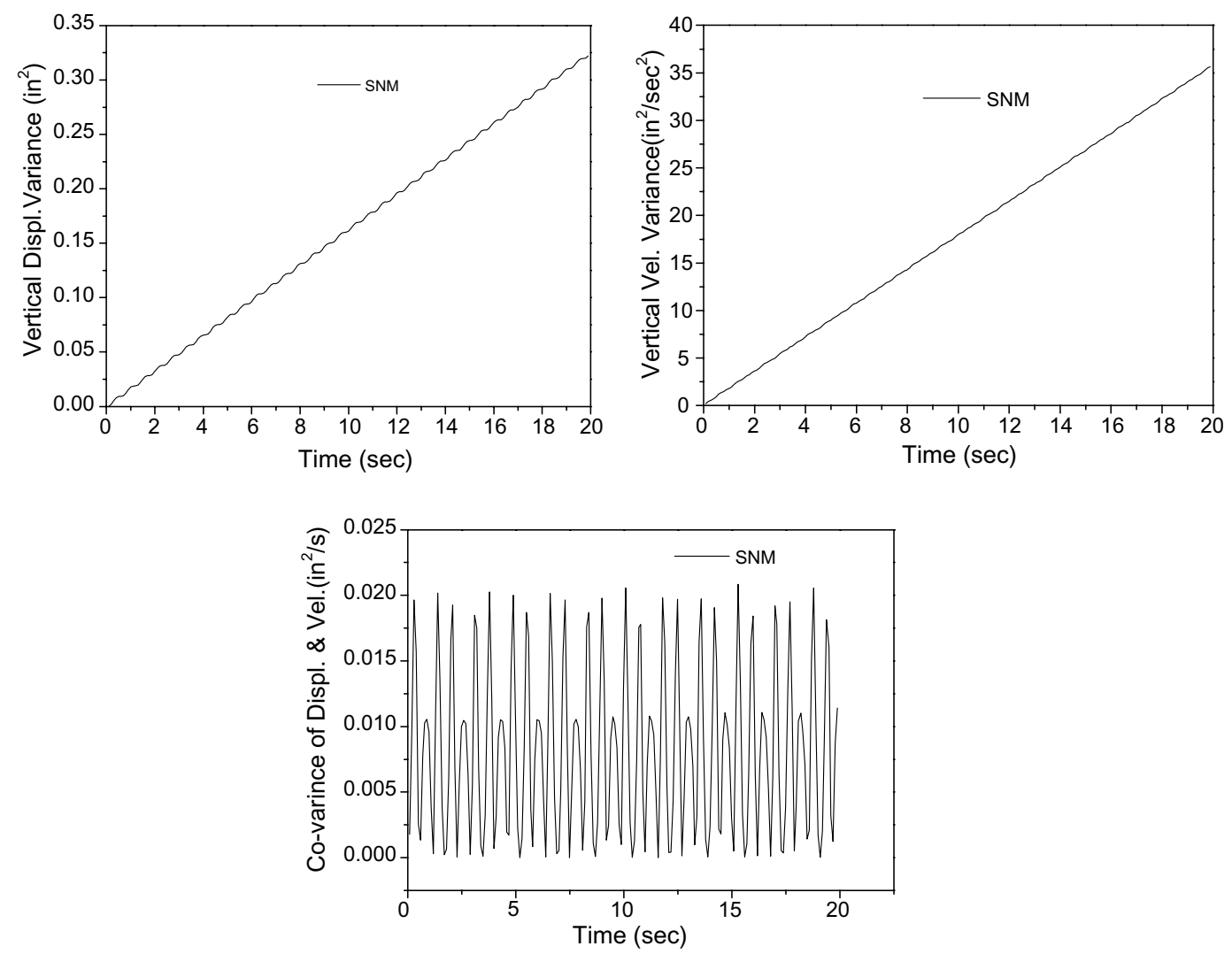

Fig. 24. Variance and covariance of displacement and velocity in the vertical direction at the free end of cantilever beam without damping $\left(a_{0}=0.0, a_{1}=0.0\right)$. 
The variance and covariance results for the response variables of the 2-DOF system (Eq. (58)) have been obtained via LSNM for two damping values of $1 \%$ and $5 \%$ and plotted in Fig. 20. The results match well with the reported results of Masri [30] and To and Orisamolu [31].

Example 6. A cantilever beam shown in Fig. 21 is analysed for response under an axial deterministic loading and a vertical stochastic load. The material and geometric properties are as shown in the figure.

The example illustrates the application of stochastic Newmark method to a simple multi-dimensional problem having total degree of freedom equal to 6 . However with fixed support condition at the node ' 1 ', there are only 3 DOF-s to be determined at node ' 2 '. As a result, the dimension of the problem is $n=3$. There are three displacements and three velocity components respectively denoted as $(u, v, \theta)^{\mathrm{T}}$ and $(\dot{u}, \dot{v}, \dot{\theta})^{\mathrm{T}}$ at node 2 . In Fig. 22, displacement response plots at the free end of the cantilever displacement beam in axial and lateral direction for two different cases, with or without the effects of axial shortening are shown without damping effects. In this example, the geometric stiffness accounts for axial shortening
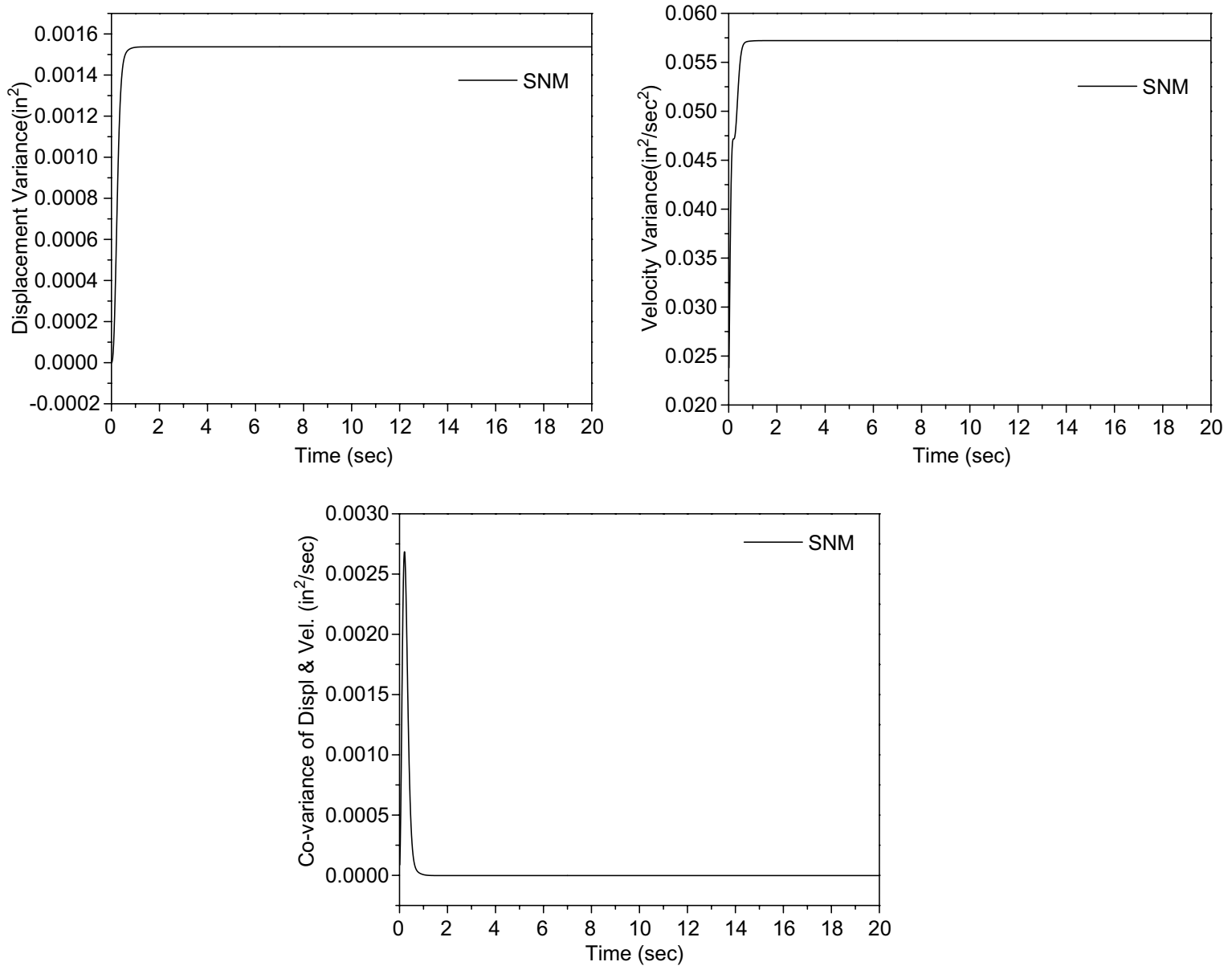

Fig. 25. Variance and covariance of displacement and velocity in the vertical direction at the free end of cantilever beam with damping $\left(a_{0}=0.05, a_{1}=0.05\right)$. 
in the beam element. Considering viscous damping of the system with assumed proportionality constants $a_{0}$ and $a_{1}$ as 0.05 each, similar displacement and velocity response at the free end in axial and vertical direction for both the cases (with and without the effects of axial shortening) are shown in Fig. 23. Variance and covariance plots for displacement and velocity in the vertical direction at the free end of the cantilever beam are shown in Figs. 24 and 25 with and without damping respectively. It is observed that displacements and velocity variance in the vertical direction (corresponding to stochastic load) of the cantilever beam grows with time when there is no damping in the system. However, with introduction of small damping in the system, variance bounds are formed, as expected.

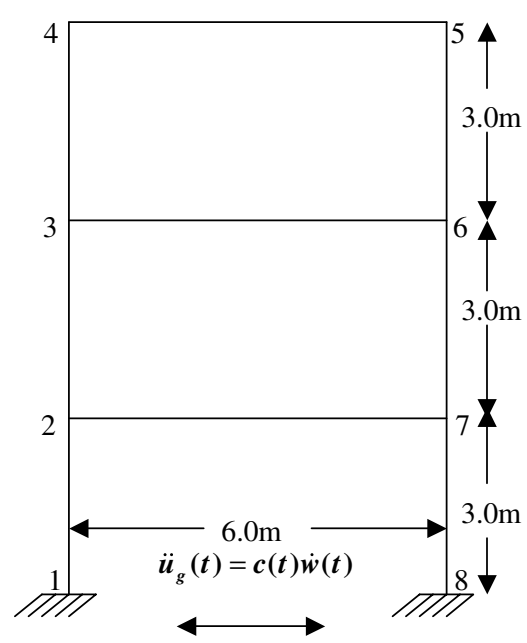

(a)

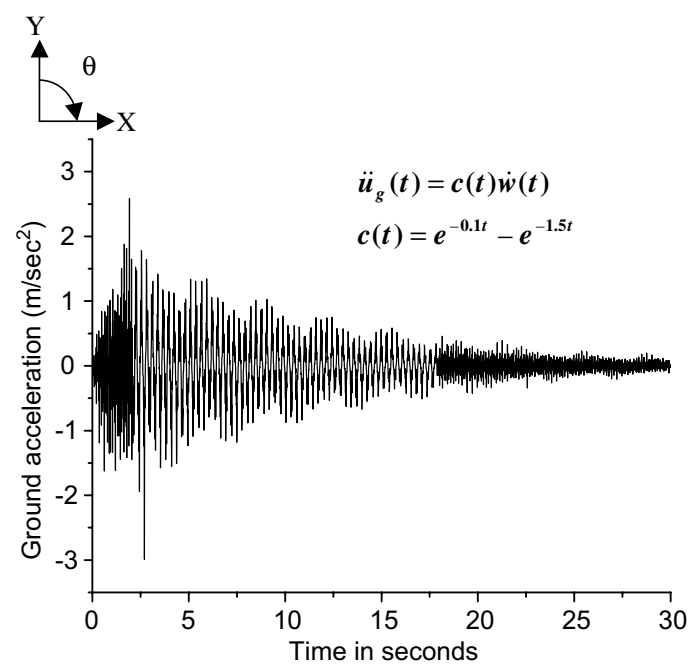

(b)

Fig. 26. Frame subjected to (a) earthquake excitation in horizontal direction and (b) ground input acceleration.
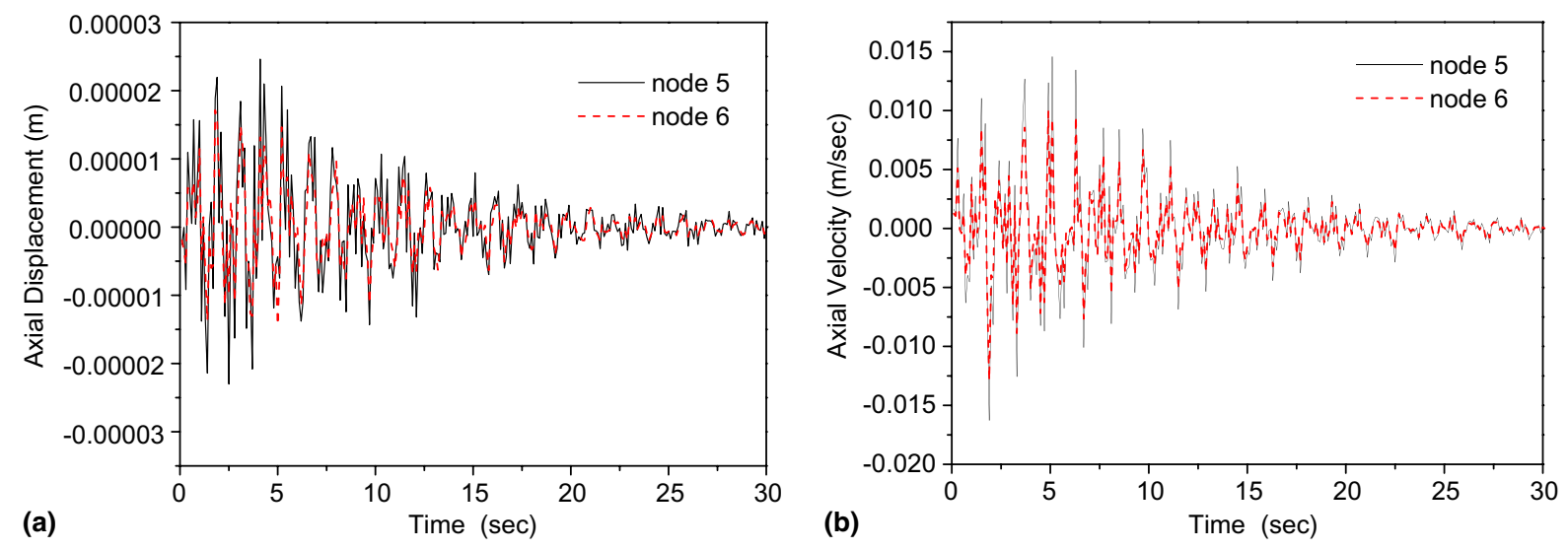

Fig. 27. Response at nodes 5 and 6 in $X$-direction: (a) Time-displacement plot and (b) time-velocity plot. 

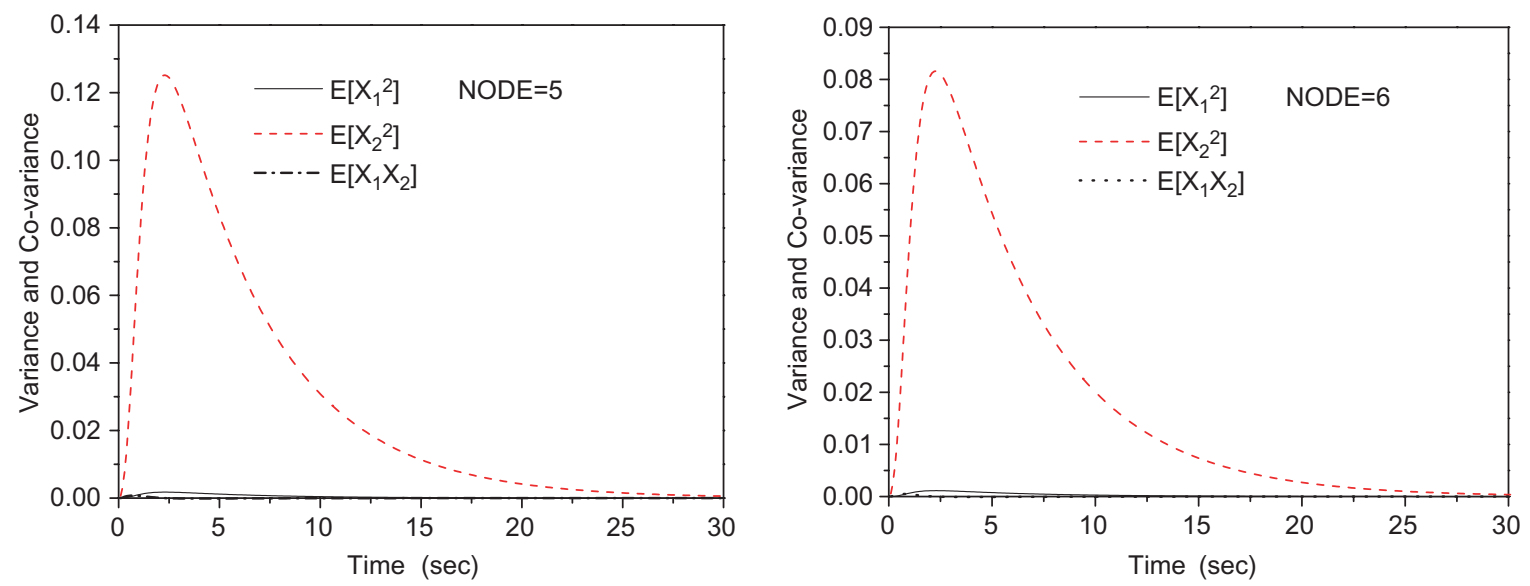

Fig. 28. Variance and co-variance at nodes 5 and 6 in $X$-direction.

Example 7. For the shear frame shown in Fig. 26, the following are the geometric and material properties: Column C.S $=0.0625 \mathrm{~m}^{2}$, Column M.I $=0.0004 \mathrm{~m}^{4}$, Beam C.S $=0.125 \mathrm{~m}^{2}$, Beam M.I $=0.003 \mathrm{~m}^{4}$, $E=3.5 \times 10^{9} \mathrm{~kg} / \mathrm{m}^{2}, \mathrm{Sp}$. Weight $=2500 \mathrm{~kg} / \mathrm{m}^{3}$.

The frame under consideration in Fig. 26(a) is subjected to horizontal ground acceleration modeled as a non-stationary stochastic process. The record of the ground acceleration is shown in Fig. 26(b). The ground acceleration is modeled as the product of modulated white noise and an enveloping function $c(t)$ [17] shown in the diagram. The displacement and velocity plots as well as their variance and co-variance plots in axial direction ( $X$-axis of frame) are shown from Figs. 27 and 28 for two typical nodes 5 and 6 of the frame. The proportionality constants $a_{0}$ and $a_{1}$ are assumed to be 0.05 in the calculation of Rayleigh damping matrix. The example further illustrates the application of LSNM to MDOF systems for response calculations.

\section{Concluding remarks}

A new family of stochastic Newmark algorithms for direct time integration of engineering dynamical systems driven by additive, multiplicative or filtered white noise processes (defined as formal derivatives of Gauss-Markov Wiener processes) is derived in this Paper. The basis of this new development is a two-parameter implicit stochastic Taylor expansion for both displacement and velocity components. Given a time step size, $h$, the displacement and velocity components are expanded up to $\mathrm{O}\left(h^{2}\right)$ and $\mathrm{O}(h)$ respectively in the lower order scheme and up to $\mathrm{O}\left(h^{3}\right)$ and $\mathrm{O}\left(h^{2}\right)$ in the higher order scheme. While both lower and higher order Newmark maps are readily adaptable for path wise solutions of linear and non-linear multi-degree-of freedom (MDOF) stochastic engineering systems, far less number of random variables need to be modelled in the lower order method, thereby making it computationally faster. The higher order method, on the other hand, is more accurate and in certain cases, especially for non-linear dynamical systems under multiplicative noise inputs, yields conspicuously more accurate results. Rigorous estimates of local and global error orders in displacement and velocity components have been included in the Paper. A detailed discussion on the exact or approximate modelling of the MSI-s has also been provided. A host of numerical illustrations on the application of these schemes for pathwise integration of a non-linear hardening Duffing oscillator under additive, multiplicative and filtered white noise excitations has been included. 
Some of these results, wherever appropriate, have been compared with some other available stochastic integration schemes.

Implementation of the stochastic Newmark integration technique based on Ito-Taylor expansion requires computation of MSI-s which is an involved task, particularly for higher order schemes. This encourages development of another single step alternative, wherein one can avoid or drastically reduce the computation of MSI-s. Such a stochastic integration scheme known as 'Locally Transversal Linearization (LTL)' method has been developed and will be discussed in another paper.

\section{Acknowledgement}

The authors must express their thankfulness to one of the referees who suggested the inclusion of the following appendices to make the revised manuscript more self-contained and readable.

\section{Appendix A. A brief introduction to Ito's formula}

\section{A.1. Wiener processes}

A $q$-dimensional Wiener process $\left\{W_{r}(t) \mid r=1, \ldots, q\right\}$ is a vector stochastic process with the following properties:

1. Normal increments: $W_{r}(t)-W_{r}(s)$ has normal distribution with mean zero and variance $(t-s)$ for every $r \in[1, q]$.

2. Independence of increments: $W_{r}(t)-W_{r}(s)$ is independent of the past, i.e., of $W_{r}(u), u \leqslant s<t$ for every $r \in[1, q]$.

3. Continuity of paths: $W_{r}(t, \omega)$ is a continuous function of time $t$ for every realization $\omega$ and for every $r \in[1, q](\omega$ is an element of the event space $\Omega)$.

4. Any two Wiener processes $W_{r}(t)$ and $W_{l}(t)$ are mutually independent stochastic processes for $r \neq l, r, l \in[1, q]$.

\section{A.2. Ito Integral}

Let $0=t_{0}^{N}<t_{1}^{N}<\cdots<t_{N}^{N}=T$ be a partition of the interval $[0, T]$ such that $\delta_{n}=\max _{i \in[0, N-1]}\left(t_{i+1}^{N}-t_{i}^{N}\right) \rightarrow$ 0 as $N \rightarrow \infty$ and $X(t)$ be a stochastic process. Then the Ito integral $\int_{0}^{T} X(t) \mathrm{d} W_{r}(t)$ is defined as:

$$
\int_{0}^{T} X(t) \mathrm{d} W_{r}(t)=\lim _{\substack{\delta_{N} \rightarrow 0 \\ N \rightarrow \infty}} \sum_{i=0}^{N-1} X\left(t_{i}^{N}\right)\left(W_{r}\left(t_{i+1}^{N}\right)-W_{r}\left(t_{i}^{N}\right)\right) .
$$

For instance, one may readily show that

$$
\int_{0}^{T} W_{r}(t) \mathrm{d} W_{r}(t)=\frac{1}{2}\left(W_{r}^{2}(T)-T\right) .
$$

In defining the Ito integral in Eq. (A.1), attention is restricted to the class of predictable stochastic processes $X(t), t \in[0, T]$ such that:

$$
\int_{0}^{T} X^{2}(t) \mathrm{d} t<\infty \quad \text { almost surely (A.s.). }
$$


Moreover, the process $X(t)$ is assumed to be $F_{t}$ measurable, where $F_{t}$ is the increasing family of $\sigma$-algebras generated by $\left\{W_{r}(s) \mid s \in[0, t) ; r \in[1, q]\right\}$. It may be be shown [5] that the Ito integral is an $N(0, \sigma)$ random variable such that:

$$
\sigma^{2}=\operatorname{Var}\left[\int_{0}^{T} X(t) \mathrm{d} W_{r}(t)\right]=\int_{0}^{T} E\left[X^{2}(t)\right] \mathrm{d} t .
$$

\section{A.3. Quadratic variation}

Given a partition $\left\{t_{i}^{N} \mid i \in[0, N]\right\}$ of the interval $[0, t]$ as before, the quadratic variation of a process $X(t)$ is defined as:

$$
[X, X](t)=\lim _{\substack{N \rightarrow \infty \\ \delta_{N} \rightarrow 0}} \sum_{i=0}^{N-1}\left|X\left(t_{i+1}^{N}\right)-X\left(t_{i}^{N}\right)\right|^{2}
$$

where the above limit is taken in probability. For instance, one may show that

$$
\left[W_{r}, W_{r}\right](t)=t
$$

and,

$$
\left[\int_{0}^{t} X(s) \mathrm{d} W_{r}(s), \int_{0}^{t} X(s) \mathrm{d} W_{r}(s)\right](t)=\int_{0}^{t} X^{2}(s) \mathrm{d} s .
$$

The above two equations provide an amazing and counter-intuitive result in the theory of stochastic processes. For instance, from an inspection of the left hand side of Eq. (A.6.1), one would normally expect it to be a random variable (for a given $t$ ). But, it turns out that it is indeed a deterministic number. It is also interesting to note that the quadratic variation of a differentiable and bounded process (as generally encountered in deterministic dynamics of many engineering systems) over any finite time interval is identically zero. For instance, the quadratic variation of each (complex valued) scalar function $X_{m}(t)=\exp (\sqrt{-1} m t)$ of the Fourier basis set $\left\{X_{m}(t), m \in Z\right\}$ in the Hilbert space of continuous and bounded functions is zero. This is probably the most conspicuous difference of a stochastic process from a deterministic (non-stochastic) one.

\section{A.4. Stochastic differential equations ( $S D E-s)$ and Ito's formula}

Consider the vector stochastic process $X(t) \in R^{n}$ governed by the SDE:

$$
\mathrm{d} X(t)=a(t, X) \mathrm{d} t+\sigma(t, X) \mathrm{d} W(t),
$$

where $W(t) \in R^{q}$ is a $q$-dimensional vector Wiener process, $\sigma(t, X) \in R^{n \times q}$ is the diffusion matrix and $a(t, X) \in R^{n}$ is the drift vector. The SDE (A.7) is expressed in an incremental (and not in a differentiated) form as $W(t)$ is not a differentiable vector stochastic process (with respect to time). Denoting the $i$ th and $j$ th scalar component processes of the vector process $X(t) \in R^{n}$ as $X_{i}(t)$ and $X_{j}(t)$ respectively, the differential quadratic variation may be shown to be:

$$
\mathrm{d}\left[X_{i}, X_{j}\right](t)=C_{i j} \mathrm{~d} t ; i, j \in[1, n],
$$


where

$$
\{C]_{n \times n}=[\sigma]_{n \times q}[\sigma]_{q \times n}^{\mathrm{T}}
$$

with the superscript ' $\mathrm{T}$ ' denoting matrix transposition.

Let $f\left(X_{1}, X_{2}, \ldots, X_{n}\right)$ be a $C^{2}$ function. Then $f\left(X_{1}, X_{2}, \ldots, X_{n}\right)$ is also an Ito process, whose stochastic differential is given by the Ito formula:

$$
\begin{aligned}
\mathrm{d} f\left(X_{1}(t), X_{2}(t), \ldots, X_{n}(t)\right)= & \sum_{i=1}^{n} \frac{\partial}{\partial X_{i}} f\left(X_{1}(t), X_{2}(t), \ldots, X_{n}(t)\right) \mathrm{d} X_{i}(t) \\
& +\frac{1}{2} \sum_{i=1}^{n} \sum_{j=}^{n} \frac{\partial^{2}}{\partial X_{i} \partial X_{j}} f\left(X_{1}(t), X_{2}(t), \ldots, X_{n}(t)\right) \mathrm{d}\left[X_{i}, X_{j}\right](t) .
\end{aligned}
$$

It is of interest to observe that for a deterministic and differentiable vector process $X(t)$, one has $\mathrm{d}\left[X_{i}, X_{j}\right](t)=0$ and thus Ito's formula reduces to the well-known relation for the total derivative:

$$
\frac{\mathrm{d} f}{\mathrm{~d} t}=\sum_{i} \frac{\partial f}{\partial X_{i}} \dot{X}_{i}
$$

\section{Appendix B. The stochastic Taylor expansion}

To start with, it is instructive to derive the deterministic form of Taylor's expansion. Let $X(t) \in R^{n}$ be a deterministic and sufficiently differentiable process governed by the ODE-s:

$$
\frac{\mathrm{d} X(t)}{\mathrm{d} t}=\dot{X}(t)=a(t, X(t)) .
$$

Let $f(t, X)$ be a scalar or a vector function (assumed to be sufficiently smooth with respect to its arguments), so that one may write:

$$
\frac{\mathrm{d}}{\mathrm{d} t} f(t, X(t))=\frac{\partial f}{\partial t}+\sum_{i=1}^{n} \frac{\partial f}{\partial X_{i}} a_{i}(t, X)
$$

Thus one has:

$$
f(t+h, X(t+h))=f(t, X)+\int_{t}^{t+h} L f(s, X(s)) \mathrm{d} s
$$

where the operator $L$ is defined as:

$$
L=\frac{\partial}{\partial t}+\sum_{i=1}^{n} a_{i}(t, X) \frac{\partial}{\partial X_{i}} .
$$

Now, letting $f(t, X(t))=X(t)$, one immediately has from Eqs. (B.3) and (B.4):

$$
X(t+h)=X(t)+\int_{t}^{t+h} a(s, X(s)) \mathrm{d} s .
$$


Further, using Eq. (B.3) for $a(s, X(s))$ in Eq. (B.5), one obtains:

$$
\begin{aligned}
X(t+h) & =X(t)+\int_{t}^{t+h}\left[a(t, X(t))+\int_{t}^{s} L a\left(s_{1}, X\left(s_{1}\right)\right) \mathrm{d} s_{1}\right] \mathrm{d} s \\
& =X(t)+a(t, X(t)) h+\int_{t}^{t+h}(t+h-s) L a(s, X(s)) \mathrm{d} s .
\end{aligned}
$$

Applying Eq. (B.3) once more to $L a(s, X(s))$ in Eq. (B.6), the following expression is readily derived:

$$
X(t+h)=X(t)+a(t, X(t)) h+L a(t, X(t)) \frac{h^{2}}{2}+\int_{t}^{t+h} \frac{(t+h-s)^{2}}{2} L^{2} a(s, X(s)) \mathrm{d} s .
$$

Continuing in this way $m$ times (i.e., by iterating $m$ times with the formula (B.3) on the integral expansion (B.5)), one arrives at the well-known Taylor expansion of $X(t+h)$ in powers of $h$ in the neighborhood of $t$ :

$$
\begin{aligned}
X(t+h)= & X(t)+a(t, X(t)) h+L a(t, X(t)) \frac{h^{2}}{2}+\cdots+L^{m-1} a(t, X(t)) \frac{h^{m}}{m !} \\
& +\int_{t}^{t+h} \frac{(t+h-s)^{m}}{m !} L^{m} a(s, X(s)) \mathrm{d} s .
\end{aligned}
$$

Given a system of SDE-s, the corresponding expansion of the stochastic process $X(t)$ in a neighborhood of $t$ is referred to as the stochastic Taylor expansion. Alternatively, such an expansion is also called the ItoTaylor (or Stratonovich-Taylor) expansion depending upon the type interpretation of stochastic integral being employed. In this paper, use has only been made of the Ito-Taylor expansion and thus the derivation of such an expansion is briefly described here. Now consider the solution $X(t) \in R^{n}$ of the system of SDE-s (A.7), which may be written in the following alternative form:

$$
\mathrm{d} X(t)=a(t, X) \mathrm{d} t+\sum_{r=1}^{q} \sigma_{r}(t, X) \mathrm{d} W_{r}(t),
$$

where the vector function $\sigma_{r}(t, X): R \times R^{n} \rightarrow R^{n}$ is the $r$ th column of the diffusion matrix $[\sigma]_{n \times q}$. In what follows, the scalar diffusion function $\sigma_{r}^{(j)}(t, X)$ would denote the $[j, r]$ th element of the matrix $[\sigma]_{n \times q}$. If $f(t, X)$ sufficiently smooth scalar or vector function, then by Ito's formula (A.10) and the formula for the differential quadratic variation (A.8), one immediately gets for $t_{0} \leqslant t \leqslant s$ :

$$
f(s, X(s))=f(t, X)+\sum_{r=1}^{q} \int_{t}^{s} \Lambda_{r} f\left(s_{1}, X\left(s_{1}\right)\right) \mathrm{d} W_{r}\left(s_{1}\right)+\int_{t}^{s} L f\left(s_{1}, X\left(s_{1}\right)\right) \mathrm{d} s_{1} .
$$

The above equation may therefore be thought of as an integral form of Ito's formula. Similar to the deterministic case, the above equation provides a formula for the stochastic increment $\Delta f=f(s, X(s))-$ $f(t, X(t))$ of the function $f$, where the operators $\Lambda_{r}$ and $L$ are given by:

$$
\begin{aligned}
& \Lambda_{r}=\sum_{j=1}^{n} \sigma_{r}^{(j)} \frac{\partial}{\partial X_{j}}, \\
& L=\frac{\partial}{\partial t}+\sum_{j=1}^{n} a_{j}(t, X) \frac{\partial}{\partial X_{j}}+\frac{1}{2} \sum_{r=1}^{q} \sum_{i}^{n} \sum_{j=1}^{n} \sigma_{r}^{(i)} \sigma_{r}^{(j)} \frac{\partial^{2}}{\partial X_{i} \partial X_{j} .}
\end{aligned}
$$


Now applying Eq. (B.10) recursively to $\Lambda_{r} f\left(s_{1}, X\left(s_{1}\right)\right)$ and $L f\left(s_{1}, X\left(s_{1}\right)\right)$, one has:

$$
\begin{aligned}
f(t+h, X(t+h))= & f(t, X)+\sum_{r=1}^{q} \Lambda_{r} f \int_{t}^{t+h} \mathrm{~d} W_{r}(s)+L f \int_{t}^{t+h} \mathrm{~d} s \\
& +\sum_{r=1}^{q} \int_{t}^{t+h}\left(\sum_{p=1}^{q} \int_{t}^{s} \Lambda_{p} \Lambda_{r} f\left(s_{1}, X\left(s_{1}\right)\right) \mathrm{d} W_{p}\left(s_{1}\right)\right) \mathrm{d} W_{r}(s) \\
& +\sum_{r=1}^{q} \int_{t}^{t+h}\left(\int_{t}^{s} L \Lambda_{r} f\left(s_{1}, X\left(s_{1}\right)\right) \mathrm{d} s_{1}\right) \mathrm{d} W_{r}(s) \\
& +\sum_{r=1}^{q} \int_{t}^{t+h}\left(\int_{t}^{s} \Lambda_{r} L f\left(s_{1}, X\left(s_{1}\right)\right) \mathrm{d} W_{r}\left(s_{1}\right)\right) \mathrm{d} s \\
& +\int_{t}^{t+h}\left(\int_{t}^{s} L^{2} f\left(s_{1}, X\left(s_{1}\right)\right) \mathrm{d} s_{1}\right) \mathrm{d} s .
\end{aligned}
$$

Just as in deterministic Taylor expansion, if Ito's formula (B.10) is applied to expand $\Lambda_{p} \Lambda_{r} f\left(s_{1}, X\left(s_{1}\right)\right), L \Lambda_{r} f\left(s_{1}, X\left(s_{1}\right)\right)$, and $\Lambda_{r} L f\left(s_{1}, X\left(s_{1}\right)\right)$, in Eq. (B.12), then the following expression results:

$$
\begin{aligned}
f(t+h, X(t+h))= & f(t, X)+\sum_{r=1}^{q} \Lambda_{r} f \int_{t}^{t+h} \mathrm{~d} W_{r}(s)+L f \int_{t}^{t+h} \mathrm{~d} s \\
& +\sum_{r=1}^{q} \sum_{i=1}^{q} \Lambda_{i} \Lambda_{r} \int_{t}^{t+h} \int_{t}^{s} \mathrm{~d} W_{r}\left(s_{1}\right) \mathrm{d} W_{i}(s) \\
& +\sum_{r=1}^{q} \sum_{i=1}^{q} \sum_{j=1}^{q} \Lambda_{j} \Lambda_{i} \Lambda_{r} \int_{t}^{t+h} \int_{t}^{s} \int_{t}^{s_{1}} \mathrm{~d} W_{j}\left(s_{2}\right) \mathrm{d} W_{i}\left(s_{1}\right) \mathrm{d} W_{r}(s) \\
& +\sum_{r=1}^{q} \Lambda_{r} L f \int_{t}^{t+h} \int_{t}^{s} \mathrm{~d} W_{r}\left(s_{1}\right) \mathrm{d} s+\sum_{r=1}^{q} L \Lambda_{r} f \int_{t}^{t+h} \int_{t}^{s} \mathrm{~d} s_{1} \mathrm{~d} W_{r}(s) \\
& +L^{2} f \int_{t}^{t+h} \int_{t}^{s} \mathrm{~d} s_{1} \mathrm{~d} s+\rho,
\end{aligned}
$$

where $\rho$ is the set of remainder terms. When written out in long hand, these terms contain expressions involving multiple integrals of third and fourth order compositions of operators $\Lambda_{r}$ and $L$. Still higher order expansions (as in the higher order Newmark method described in this paper) may be derived by iterating the error terms $\rho$ using Eq. (B.10), i.e., the integral form of Ito's formula. It is now clear that a distinguishing feature of the Ito-Taylor expansion vis-à-vis the deterministic Taylor expansion is that the former has multiple integrals of the forms $\int_{t}^{t+h} \int_{t}^{s} \mathrm{~d} W_{r}\left(s_{1}\right) \mathrm{d} W_{i}(s), \int_{t}^{t+h} \int_{t}^{s} \int_{t}^{s_{1}} \mathrm{~d} W_{r}\left(s_{12}\right) \mathrm{d} W_{i}\left(s_{1}\right) \mathrm{d} W_{j}(s)$, $\int_{t}^{t+h} \int_{t}^{s} \mathrm{~d} W_{r}\left(s_{1}\right) \mathrm{d} s$ etc. These integrals involve increments of scalar Wiener components $\mathrm{d} W_{r}(t)$ and hence are referred to as multiple stochastic integrals (MSI-s).

\section{References}

[1] T. Belytschko, T.J.R. Hughes (Eds.), Computational Methods for Transient Analysis, North Holland, Dordrecht, 1983.

[2] P. Bernard, G. Fleury, Stochastic Newmark scheme, Probab. Eng. Mech. 17 (2002) 45-61.

[3] T.K. Caughey, H.J. Stumpf, Transient response of a dynamic system under random excitation, ASME J. Appl. Mech. 28 (1961) $563-566$.

[4] T.C. Gard, Introduction to Stochastic Differential Equations, Marcel Dekker Inc., 1988.

[5] F.C. Klebaner, Introduction to Stochastic Calculus with Applications, Imperial College Press, London, UK, 2001. 
[6] P.E. Kloeden, E. Platen, Higher order implicit strong numerical schemes for stochastic differential equations, J. Stat. Phys. 66 (1992) 283-314.

[7] P.E. Kloeden, E. Platen, Numerical Solution of Stochastic Differential Equations, Springer, Berlin, 1999.

[8] B. Miao, Direct integration variance prediction of random response of non-linear systems, Comput. Struct. 46 (6) (1993) $179-983$.

[9] G.N. Milstein, Numerical Integration of Stochastic Differential Equations, Kluwer Academic Publishers, Dordrecht, 1995.

[10] G.N. Milstein, Yu.M. Repin, M.V. Tretyakov, Symplectic integration of hamiltonian systems with additive noise, SIAM J. Num. Anal. 39 (6) (2002) 2066-2088.

[11] R.M. Miranda, Ferencz, T.J.R. Hughes, An improved implicit-explicit time integration method for structural dynamics, Int. J. Earthquake Engrg. Struct. Dyn. 18 (1989) 643-655.

[12] N.M. Newmark, A method for computation of structural dynamics, J. Engrg. Mech. (ASCE) 85 (1959) 67-94.

[13] Y. Ohtori, Spencer Jr. F. Billie, Semi-implicit integration algorithm for stochastic analysis of multi-degree-of-freedom structures, J. Engrg. Mech. ASCE 128 (6) (2002) 635-643.

[14] J. Qiang, S. Habib, Second-order stochastic leapfrog algorithm for multiplicative noise Brownian motion, Phys. Rev. E 62 (5) (2000).

[15] D. Roy, Explorations of the phase space linearization method for deterministic and stochastic non-linear dynamical systems, Int. J. Nonlinear Dyn. 23 (2000) 225-258.

[16] D. Roy, M.K. Dash, A stochastic Newmark method for engineering dynamical systems, J. Sound Vibr. 249 (1) (2002) 83-100.

[17] M. Shinozuka, Y. Sato, Simulation of non-stationary random processes, J. Engrg. Mech. Div. ASCE 93 (EM4) (1967) 11-40.

[18] C.W.S. To, The stochastic central difference method in structural dynamics, Comput. Struct. 23 (6) (1986) 813-818.

[19] C.W.S. To, Direct integration operators and their stability for random response of multi-degree-of-freedom systems, Comput. Struct. 30 (4) (1988) 865-874.

[20] C.W.S. To, Recursive expressions for a random response of non-linear systems, Comput. Struct. 29 (3) (1988) $451-457$.

[21] C.W.S. To, Parametric effects on time step of the stochastic central difference method, J. Sound Vibr. 137 (3) (1990) 523-526.

[22] C.W.S. To, A stochastic version of the Newmark family of algorithms for discretized dynamic systems, Comput. Struct. 44 (3) (1992) 667-673.

[23] A. Tocino, R. Ardanuy, Runge-Kutta methods for numerical solution of stochastic differential equations, J. Computat. Appl. Math. 138 (2002) 219-241.

[24] S.W. Zhang, H.H. Zhao, Effects of time step in stochastic central difference method, J. Sound Vibr. 159 (1) (1992) $182-188$.

[25] O.C. Zienkiewicz, R.L. Taylor, The Finite Element Method, vol. 2, McGraw Hill, UK, 1991.

[26] W. Rumelin, Numerical treatment of stochastic differential equations, SIAM J. Numer. Anal. 19 (1982) $604-613$.

[27] E.J. McShane, Stochastic Calculus and Stochastic Models, Academic Press, New York, 1974.

[28] Y. Saito, T. Mitsui, Stability analysis of numerical schemes for stochastic differential equations, SIAM J. Numer. Anal. 33 (1996) $2254-2267$.

[29] C.C. Chang, Numerical solution of stochastic differential equations with constant diffusion coefficients, Math. Comp. 49 (1987) $523-542$.

[30] S.F. Masri, Response of a multi-degree-of-freedom system to non-stationary random excitation, ASME J. Appl. Mech. 45 (1978) 649-656.

[31] C.W.S. To, R.I. Orisamolu, Response of a two-degree-of-freedom system to random disturbances, Computers and Structures 25 (1987) 311-320.

[32] L. Zhang, J.W. Zu, Z. Zheng, The stochastic Newmark algorithm for random analysis of multi-degree-of-freedom non-linear systems, Computers and Structures 70 (1999) 557-568. 